\title{
FEASIBILITY OF NONINVASIVE THERMOMETRY IN HYPERTHERMIA REGIME USING HARMONICS GENERATED BY NONLINEAR ULTRASOUND WAVE PROPAGATION
}

\author{
by \\ Borna Maraghechi \\ M.Sc. Laurentian University, Sudbury, Canada, 2012 \\ B.Sc. Amirkabir University of Technology, Tehran, Iran, 2009 \\ A dissertation \\ presented to Ryerson University \\ in partial fulfillment of the \\ requirements for the degree of \\ Doctor of Philosophy \\ in the Program of \\ Biomedical Physics
}

Toronto, Ontario, Canada, 2016

(C) Borna Maraghechi, 2016 


\section{Author's Declaration}

I hereby declare that I am the sole author of this dissertation. This is a true copy of the dissertation, including any required final revisions, as accepted by my examiners.

I authorize Ryerson University to lend this dissertation to other institutions or individuals for the purpose of scholarly research

I further authorize Ryerson University to reproduce this dissertation by photocopying or by other means, in total or in part, at the request of other institutions or individuals for the purpose of scholarly research.

I understand that my dissertation may be made electronically available to the public.

Borna Maraghechi 


\title{
Abstract
}

\section{Feasibility of noninvasive thermometry in hyperthermia regime using harmonics generated by nonlinear ultrasound wave propagation}

\author{
Borna Maraghechi \\ Doctor of Philosophy, Biomedical Physics \\ Ryerson University, 2016
}

Hyperthermia is a cancer treatment modality that could be delivered as a stand-alone treatment or in conjunction with chemotherapy or radiation therapy. Noninvasive and real-time temperature monitoring of the heated tissue improves the efficacy and safety of the treatment. Ultrasound-based thermometry requires a temperature-sensitive acoustic parameter that can be used to estimate the temperature by tracking changes in that parameter during heating. This dissertation describes the experiments and simulations performed to obtain the temperature dependence of acoustic harmonics generated by nonlinear ultrasound propagation in several media including: water, an attenuating tissue-mimicking liquid, ex vivo bovine muscle tissues, and tissue-mimicking gel phantoms. The mechanisms of action of harmonic generation in water and in the attenuating liquid, made by a mixture of $90 \%$ glycerol and $10 \%$ water (by volume), as a function of temperature at various frequencies have been investigated using a temperature dependent Khokhlov-Zabolotskaya-Kuznetsov (KZK) nonlinear acoustic wave propagation model. The simulation results were compared with and validated by measurements. In water, the harmonic amplitudes decrease with increasing the temperature at low frequencies $(1$ and 3.3 $\mathrm{MHz}$ ), while the opposite temperature dependence was observed at higher frequencies (13 and $20 \mathrm{MHz}$ ). The harmonic generation significantly increased with temperature in the tissue- 
mimicking liquid at both frequencies of 5 and $13 \mathrm{MHz}$. The temperature dependence of harmonics in tissue-mimicking gel phantoms and ex vivo bovine muscle tissues were measured using a commercial high-frequency ultrasound imaging system, and a new noninvasive ultrasound-based thermometry has been developed that is based on the backscattered energy of the harmonics. The sensitivity of this new thermometry technique to medium's motion was studied and compared with the conventional echo-shift thermometry technique. Based on this study, it is suggested that noninvasive temperature estimation is feasible using acoustic harmonics with lower sensitivity to motion artifacts compared to the conventional echo-shift technique. 


\section{Acknowledgements}

First and foremost, I would like to express my sincere gratitude to my supervisors Dr. Jahan Tavakkoli and Dr. Michael C. Kolios for providing guidance, support and training throughout my studies. My gratitude extends to the members of the supervisory committee, Dr. Carl Kumaradas and Dr. Yuan $\mathrm{Xu}$, for their time and insightful comments.

I would like to acknowledge all the people that have assisted me during this work and which made all of this possible. I would like to show my appreciation to Mr. Arthur Worthington for his technical support and members of the Kolios lab, especially Dr. Lauren Wirtzfeld, for their continuous help. Technical help and guidance from Dr. Mojtaba H. Hasani is highly appreciated. I also wish to thank all my colleagues who are also my close friends, Pooya Sobhe Bidari, Elyas Shaswary and Amin Jafari Sojahrood for their help in many different ways.

I am especially grateful to my parents for supporting me throughout my studies, and to my loving, encouraging, and patient wife, Noushin who have helped me endure the highs and lows of all these years. Thanks to my friends, Amir Nobakhti, Refigh Masoud, Peyman Alizadeh, Elias Khorasanizadeh and Hossein Mirshahi for always being there when I have needed them. 


\section{Table of Contents}

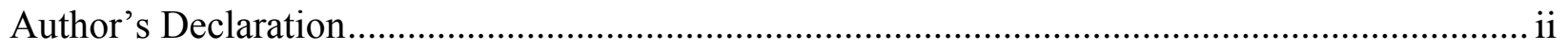

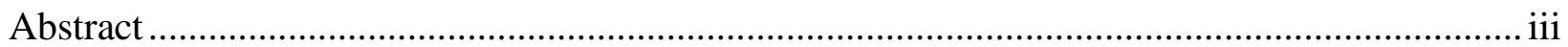

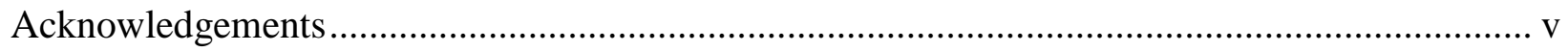

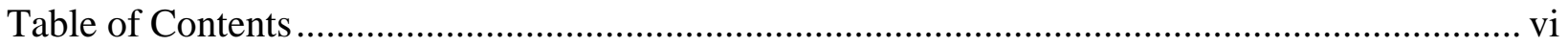

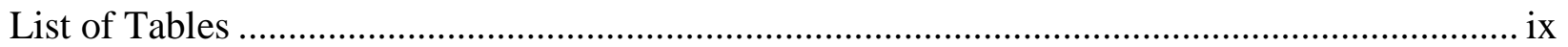

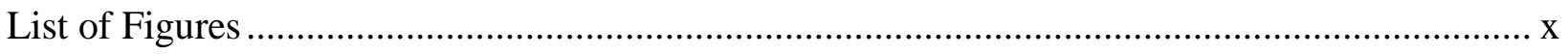

List of Symbols and Abbreviations.................................................................................. .

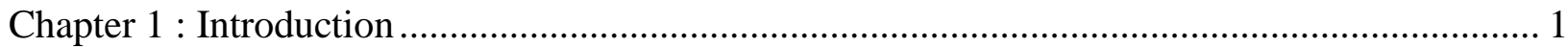

1.1 Heat and its Applications in Medicine and Biology ...................................................... 1

1.2 Tissue Heating Modalities ....................................................................................... 4

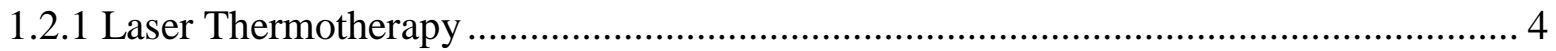

1.2.2 Gold Nanoparticle Thermotherapy ....................................................................... 5

1.2.3 Microwave Thermotherapy ………......................................................................... 6

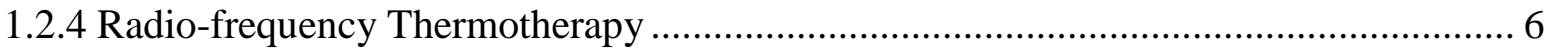

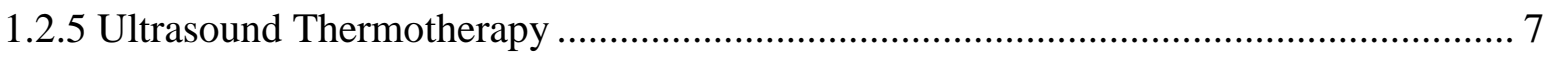

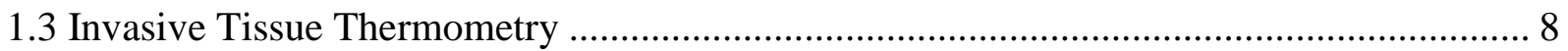

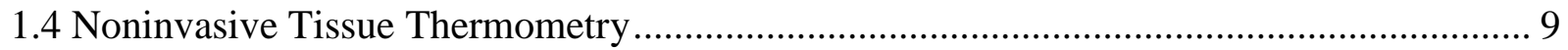

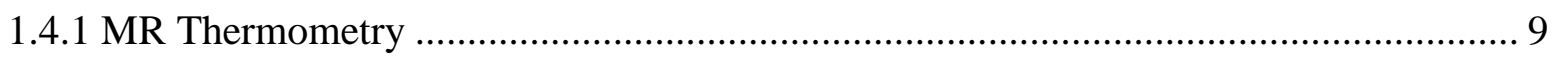

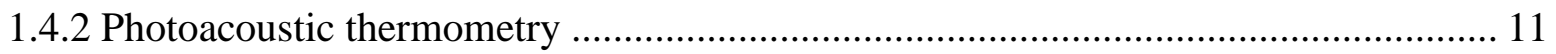

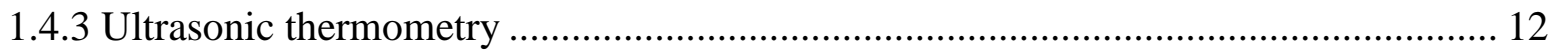

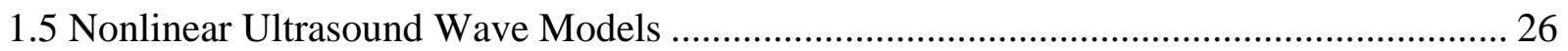

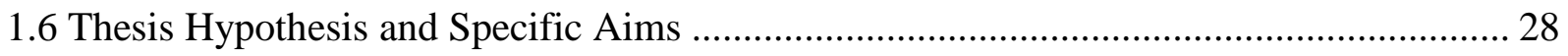

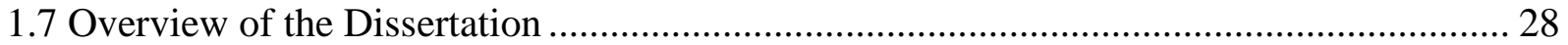

Chapter 2 : Temperature dependence of acoustic harmonics generated by nonlinear ultrasound wave propagation in water at various frequencies ......................................................................... 30

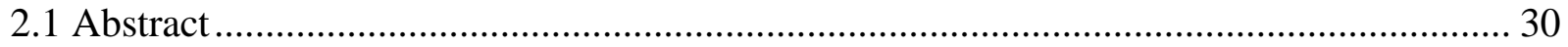

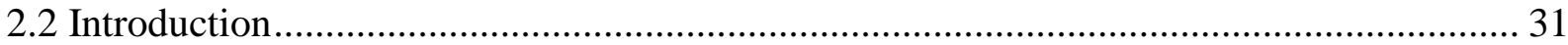




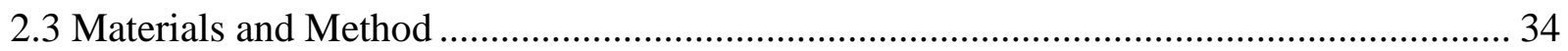

2.3.1 Temperature Dependent Nonlinear Ultrasound Wave Propagation Model .................. 34

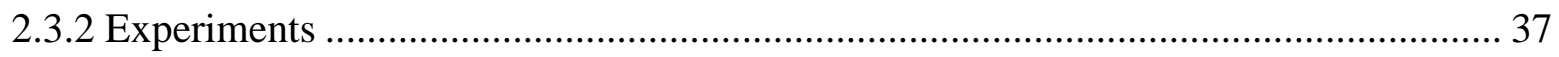

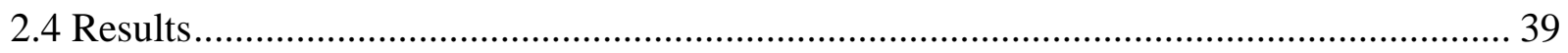

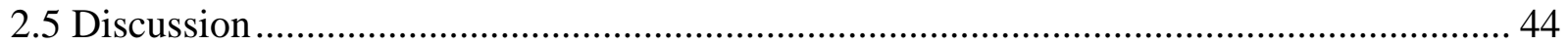

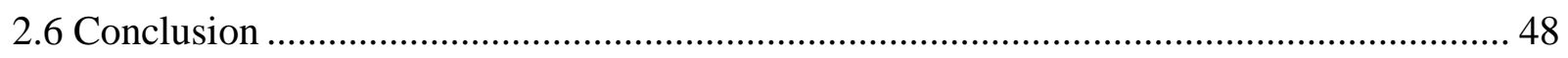

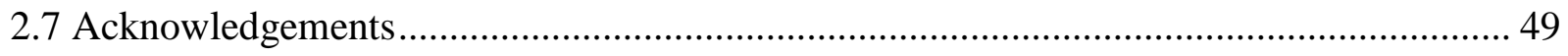

Chapter 3 : Temperature dependence of acoustic harmonics in a lossy liquid: measurement and

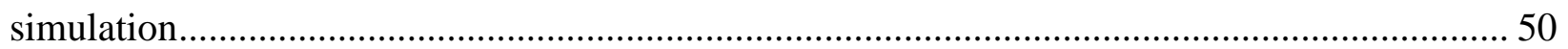

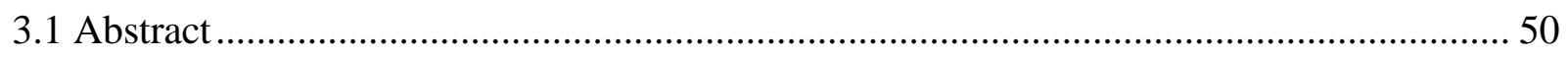

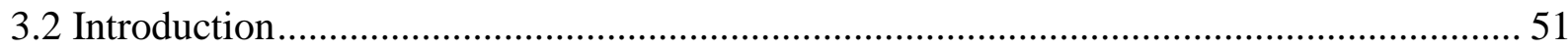

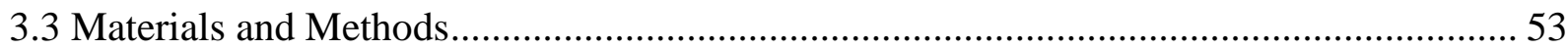

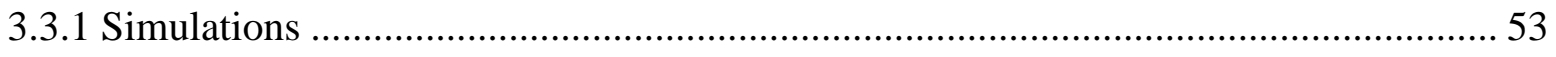

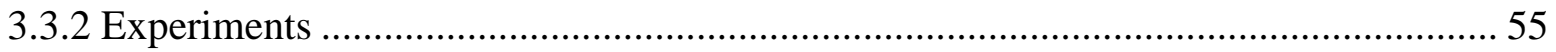

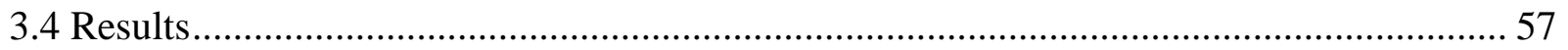

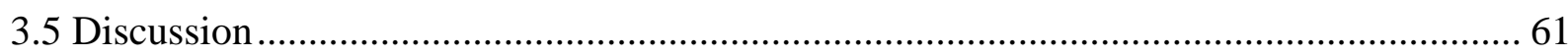

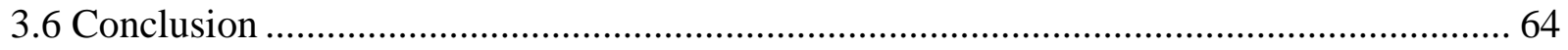

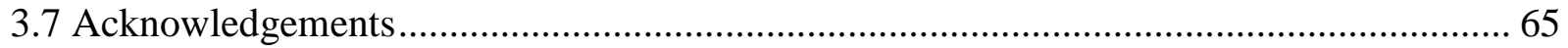

Chapter 4 : Temperature dependence of acoustic harmonics generated by nonlinear ultrasound beam propagation in ex vivo tissue and tissue-mimicking phantoms .......................................... 66

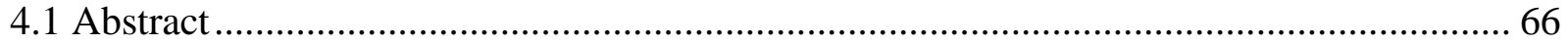

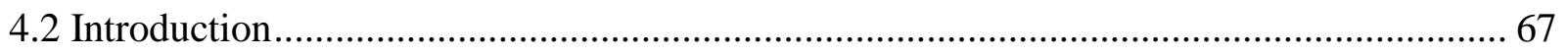

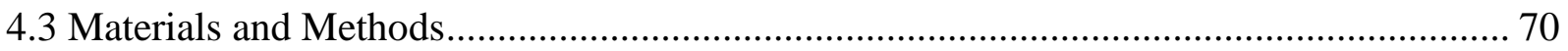

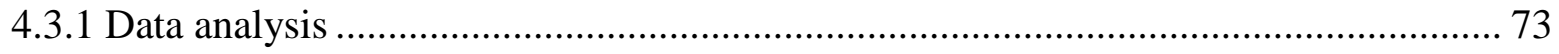

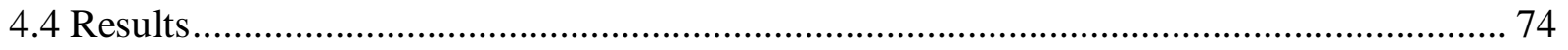

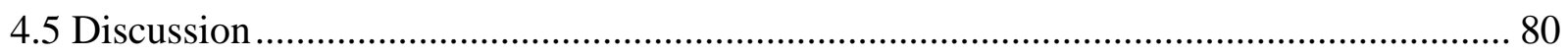

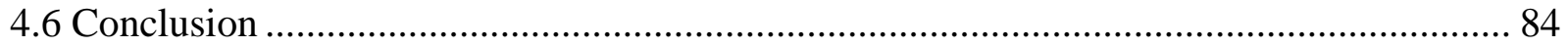

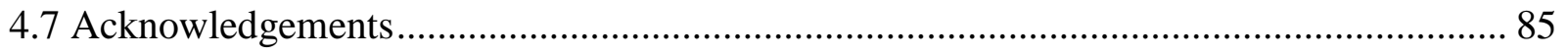

Chapter 5 : Feasibility of noninvasive temperature estimation using acoustic harmonics in tissue-

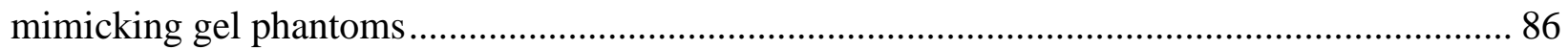

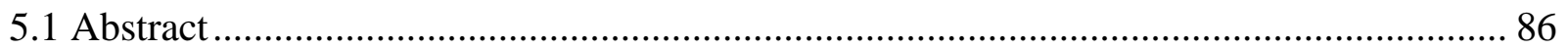




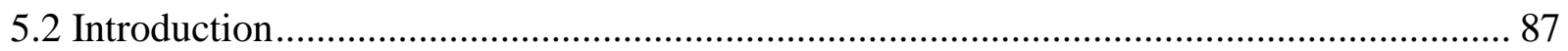

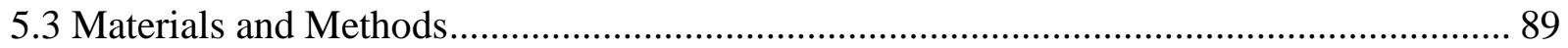

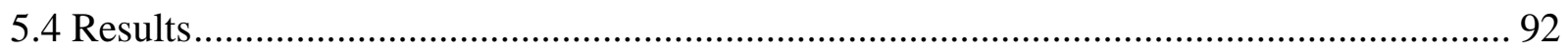

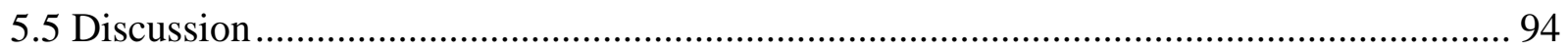

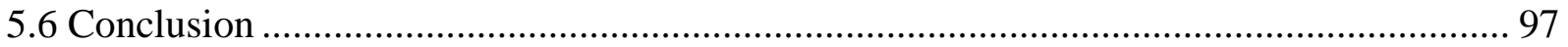

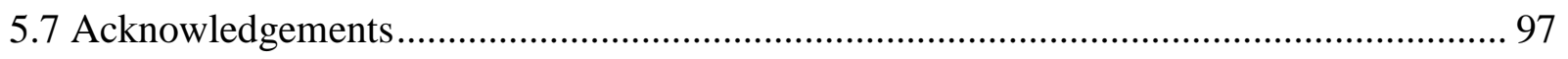

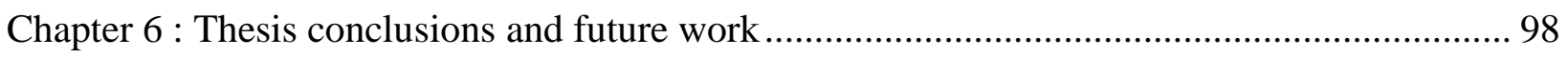

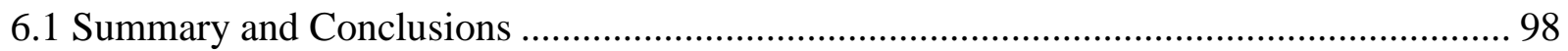

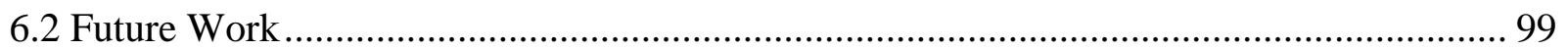

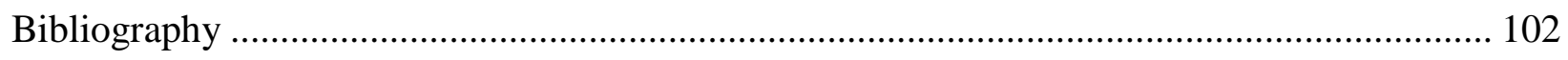




\section{List of Tables}

Table 4-1: Percentage increase in $\mathrm{p} 1, \mathrm{p} 2, \mathrm{p} 3$ and the ratios $\mathrm{p} 2 / \mathrm{p} 1$ and $\mathrm{p} 3 / \mathrm{p} 1$ as the temperature was increased from $26^{\circ} \mathrm{C}$ to $46^{\circ} \mathrm{C}$ in tissue-mimicking gel phantoms and ex vivo tissue samples.

Table 4-2: Percentage increase in E, E1, E2, E3 and the ratios E2/E1 and E3/E1 as the temperature was increased from $26^{\circ} \mathrm{C}$ to $46^{\circ} \mathrm{C}$ in tissue-mimicking gel phantoms and ex vivo tissue samples. 79

Table 5-1: The mean and the standard deviation of calculated SNR and CNR values for temperature and gradient maps without the presence of vibration in the phantom............. 96

Table 5-2: The mean and the standard deviation of calculated SNR and CNR values for temperature and gradient maps with the presence of vibration in the phantom 96 


\section{List of Figures}

Figure 1-1: Effect of ultrasound hyperthermia on TSL uptake at tumor region [5] ...................... 2

Figure 1-2: Effect of thermal ablation therapy on non-TSL uptake at tumor region [5]............... 4

Figure 1-3: Attenuation coefficient versus temperature for dog muscle, liver, and kidney in vitro [56].

Figure 1-4: (a) The adiabatic compressibility and (b) the speed of sound in water as a function of temperature [53]

Figure 1-5: (a) The dotted line presents a pulse at $0 \mathrm{~cm}$ and the solid line presents the distorted pulse due to nonlinearity at $10 \mathrm{~cm}$ in water. (b) The dotted line represents the normalized frequency spectrum of the signal at $0 \mathrm{~cm}$ and the solid line represents the normalized frequency spectrum of the distorted pulse at $10 \mathrm{~cm}$ which has generated multiple

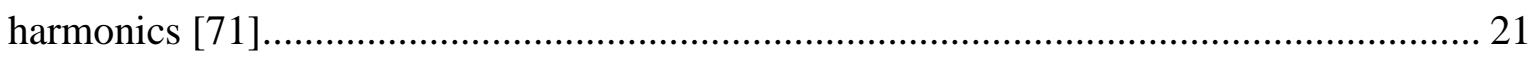

Figure 1-6: Temperature dependence of B/A of porcine (a) fat and (b) liver [52]..................... 24

Figure 1-7: Simulated normalized frequency spectrum of an acoustic plane wave at distance $x=$ $0.1 \mathrm{~m}$ in glycerol at temperatures $T=40^{\circ} \mathrm{C}, 50^{\circ} \mathrm{C}$ and $60^{\circ} \mathrm{C}$. The fundamental frequency is $1 \mathrm{MHz}[51]$.

Figure 2-1: The (a) attenuation coefficient [53], (b) speed of sound [53], (c) density [51], and (d) $\mathrm{B} / \mathrm{A}[84]$ as empirical functions of temperature in water. 37

Figure 2-2: Schematic of the experimental setup at $3.3 \mathrm{MHz}$ 39

Figure 2-3: Simulated normalized focal pressures and the magnitude of their spectra for pulses with transmit frequencies of (a) and (b) $3.3 \mathrm{MHz}$ and (c) and (d) at $13 \mathrm{MHz}$, respectively at the initial temperature $\left(26^{\circ} \mathrm{C}\right)$ 
Figure 2-4: Measured normalized focal pressures and the magnitude of their spectra for pulses with transmit frequencies of (a) and (b) $3.3 \mathrm{MHz}$ and (c) and (d) $13 \mathrm{MHz}$, respectively at the initial temperature $\left(26^{\circ} \mathrm{C}\right)$

Figure 2-5: Measured (dotted) and simulated (solid) normalized pressure amplitudes of the lowest three harmonic components along the axis of the beam with transmit frequencies of (a) $3.3 \mathrm{MHz}$ and (b) $13 \mathrm{MHz}$ at the initial temperature $\left(26^{\circ} \mathrm{C}\right)$. 42

Figure 2-6: Simulated (left column) and measured (right column) changes in (a) and (b) $p_{1}$, (c) and (d) $p_{2}$, (e) and (f) $p_{3}$ as a function of temperature with respect to the initial temperature $\left(26^{\circ} \mathrm{C}\right.$ ) for pulses with transmit frequencies of 1, 3.3, 13 and $20 \mathrm{MHz}$. Measurements (right column) were only performed at two frequencies of 3.3 and $13 \mathrm{MHz}$. The error bars represent the standard deviation of 12 independent trials. 43

Figure 2-7: Simulated (left column) and measured (right column) changes in (a) and (b) $p_{2} / p_{1}$, (c) and (d) $p_{3} / p_{1}$ as a function of temperature with respect to the initial temperature $\left(26^{\circ} \mathrm{C}\right)$ for pulses with transmit frequencies of 1, 3.3, 13 and $20 \mathrm{MHz}$. Measurements (right column) were only performed at two frequencies of 3.3 and $13 \mathrm{MHz}$. The error bars represent the standard deviation of 12 independent trials.

Figure 2-8: The percentage changes in (a) the nonlinear parameter $(N)$, (b) the absorption parameter $(A)$, (c) the Gol'dberg number $(\Gamma)$ and $(\mathrm{d})$ the focusing gain $(G)$ as a function of temperature for pulses with transmit frequencies of 1, 3.3, 13 and $20 \mathrm{MHz}$. 45

Figure 2-9: The percentage change of $\left[\beta /\left(\alpha \rho_{0} c_{0}{ }^{3}\right)\right]^{n-1}$ as a function of temperature for the second $(n=2)$, third $(n=3)$ and fourth $(n=4)$ harmonics at $13 \mathrm{MHz}$ transmit pulse frequency. .... 47

Figure 3-1: The values of (a) attenuation coefficient [90], (b) speed of sound [53, 91], (c) density $[91,51]$ and (d) B/A [91, 84] as a function of temperature in a 90/10 mixture of glycerol and water. 55

Figure 3-2: Schematic of the experimental setup at $13 \mathrm{MHz}$. 57

Figure 3-3: Simulated (left column) and measured (right column) normalized axial profiles of (a) and (b) $p_{1}$, (c) and (d) $p_{2}$, (e) and (f) $p_{3}$ as a function of temperature with respect to the 
maximum temperature $\left(46^{\circ} \mathrm{C}\right)$ at $5 \mathrm{MHz}$. The error bars represent the standard deviation of 6 independent trials. The correspondence between plots and temperatures is shown in panel (e). The magnitude of the harmonics increases monotonically with temperature from $26^{\circ} \mathrm{C}$ to $46^{\circ} \mathrm{C}$ 58

Figure 3-4: Simulated (left column) and measured (right column) normalized axial profiles of (a) and (b) $p_{1}$, (c) and (d) $p_{2}$, (e) and (f) $p_{3}$ as a function of temperature with respect to the maximum temperature $\left(46^{\circ} \mathrm{C}\right)$ at $13 \mathrm{MHz}$. The error bars represent the standard deviation of 6 independent trials. The correspondence between plots and temperatures is shown in panel (a) 59

Figure 3-5: Simulated (dotted) and measured (solid) changes in the peak values of (a) and (b) $p_{1}$, (c) and (d) $p_{2}$, (e) and (f) $p_{3}$ as a function of temperature in a 90/10 glycerol/water mixture at $5 \mathrm{MHz}$ (left column) and $13 \mathrm{MHz}$ (right column). The error bars represent the standard deviation of six trials.

Figure 3-6: Simulated (dotted) and measured (solid) changes in the peak values of (a) and (b) $p_{2} / p_{1}$, and (c) and (d) $p_{3} / p_{1}$ as a function of temperature in a $90 / 10$ glycerol/water mixture at $5 \mathrm{MHz}$ (left column) and $13 \mathrm{MHz}$ (left column). The error bars represent the standard deviation of six trials. 61

Figure 3-7: The percentage changes of (a) the nonlinear parameter $(N)$, (b) the absorption parameter $(A)$, (c) the Gol'dberg number $(\Gamma)$, and (c) the focusing gain $(G)$ as a function of temperature in a 90/10 glycerol/water mixture at frequencies 5 and $13 \mathrm{MHz}$. 62

Figure 4-1: Schematic of the experimental setup. 72

Figure 4-2: The averaged (100 RF lines) values of (a) and (b) $p_{1}$, (c) and (d) $p_{2}$, (e) and (f) $p_{3}$ in tissue-mimicking gel phantom (left column) and ex vivo bovine muscle tissue (right column) at temperatures of $26^{\circ} \mathrm{C}, 34^{\circ} \mathrm{C}, 38^{\circ} \mathrm{C}$ and $46^{\circ} \mathrm{C}$.

Figure 4-3: Changes in (a) $p_{1}$, (b) $p_{2}$, (c) $p_{3}$ and the ratios (d) $p_{2} / p_{1}$ and (e) $p_{3} / p_{1}$ as a function of temperature in tissue-mimicking gel phantoms with respect to the initial temperature $\left(26^{\circ} \mathrm{C}\right)$. The error bars represent the standard deviation of 5 trials. 76 
Figure 4-4: Changes in (a) $p_{1}$, (b) $p_{2}$, (c) $p_{3}$ and the ratios (d) $p_{2} / p_{1}$ and (e) $p_{3} / p_{1}$ as a function of temperature in ex vivo bovine muscle tissues with respect to the initial temperature $\left(26^{\circ} \mathrm{C}\right)$. The error bars represent the standard deviation of 6 trials. 77

Figure 4-5: Changes in (a) $E$, (b) $E_{1}$, (c) $E_{2}$, (d) $E_{3}$ and the ratios (e) $E_{2} / E_{1}$ and (f) $E_{3} / E_{1}$ at the focal region as a function of temperature in tissue-mimicking gel phantoms with respect to the initial temperature $\left(26^{\circ} \mathrm{C}\right)$. The error bars represent the standard deviation of 5 trials. 78

Figure 4-6: Changes in (a) $E$, (b) $E_{1}$, (c) $E_{2}$, (d) $E_{3}$ and the ratios (e) $E_{2} / E_{1}$ and (f) $E_{3} / E_{1}$ at the focal region as a function of temperature in ex vivo bovine muscle tissues with respect to the initial temperature $\left(26^{\circ} \mathrm{C}\right)$. The error bars represent the standard deviation of 6 trials. 79

Figure 4-7: Changes in (a) $p_{1}$, (b) $E_{1}$, (c) $p_{2}$, (d) $E_{2}$, (e) $p_{3}$ and (f) $E_{3}$ at $44^{\circ} \mathrm{C}$ as a function of time in ex vivo bovine muscle tissues with respect to the initial time (40 minutes). The error bars represent the standard deviation of 4 trials 80

Figure 5-1: Schematic of the experimental setup. 90

Figure 5-2: Estimated 2D temperature maps in tissue mimicking gel phantom obtained using CBE1 (left), CBE2 (middle), and CBE3 (right) while the temperature was cooling down from $46^{\circ} \mathrm{C}$ to $26^{\circ} \mathrm{C}$ and no vibration was present in the phantom. The temperatures measured by the inserted thermocouple were $31,38,42$ and $46^{\circ} \mathrm{C}$ at the center of heated region 93

Figure 5-3: Estimated 2D temperature maps in tissue mimicking gel phantom obtained using $\mathrm{CBE} 1$ (left), $\mathrm{CBE} 2$ (middle), and CBE3 (right) while the temperature was elevated from 26 to $46^{\circ} \mathrm{C}$ with the presence of vibration in the phantom. The temperatures measured by the inserted thermocouple were $31,38,42$ and $46^{\circ} \mathrm{C}$ at the center of heated region. 93

Figure 5-4: 2D normalized gradient maps in tissue mimicking gel phantom obtained using the conventional echo-shift technique without (left) and with (right) presence of vibration in the phantom. The temperatures measured by the inserted thermocouple were 31, 38, 42 and $46^{\circ} \mathrm{C}$ at the center of heated region. 94 


\section{List of Symbols and Abbreviations}

\section{Chapter 1}

$T$

$t$

$t_{43}$

$\omega^{\prime}$

$\gamma^{\prime}$

$B_{\text {loc }}$

$B_{0}$

$\Gamma^{\prime}$

$\alpha_{V}$

$c_{0}$

$C_{p}$

$\bar{I}$

$\alpha_{s}$

$\alpha_{a}$

$\alpha$

$\alpha_{c}$

$\omega$

$\kappa$

$\eta_{s}$

$\gamma$

$\mathrm{K}$

$\eta_{b}$

$\alpha_{t}$

$\rho_{0}$

$\lambda$

$\alpha_{L}$

$s$

$B / A$

$\beta$

$v$

$F_{0}$

$H_{i}$

$\tau$
Temperature

Time

Cumulative equivalent minutes at $43^{\circ} \mathrm{C}$

Resonance frequency

Gyromagnetic ratio

Local magnetic field

Magnetic flux density

Grüneisen parameter

Thermal volume expansion of the medium

Speed of sound

Specific heat capacity at a constant pressure

Averaged intensity

Scattering amplitude attenuation coefficient

Absorption amplitude attenuation coefficient

Amplitude attenuation coefficient

Classical absorption of sound in fluids

Angular frequency

Adiabatic compressibility

Shear viscosity

Ratio of specific heats

Thermal conductivity of the medium

Bulk viscosity

Theoretical absorption coefficient in liquids

Medium density

Thermal coefficient of speed of sound

Linear coefficient of thermal expansion of the medium

Entropy

Nonlinearity of medium

Coefficient of nonlinearity

Particle velocity

Pressure amplitude of the fundamental frequency

Pressure amplitude of the $\mathrm{i}^{\text {th }}$ harmonic

Retarded time 


$\begin{array}{ll}\delta & \text { Diffusivity of sound } \\ k & \text { Wave number } \\ a & \text { Source radius }\end{array}$

\section{Chapters 2 and 3}

$T$

$f_{0}$

$P$

$p$

$p_{0}$

$\sigma$

$a$

$b$

$\omega_{0}$

$X$

Y

G

$Z_{0}$

$c_{0}$

$\rho_{0}$

$\tau$

$t^{\prime}$

$N$

$\bar{Z}$

$f(t)$

$g(x, y)$

$\xi(x, y)$

$G_{x}$

$G_{y}$

$n$

$B / A$

$\Gamma$

$p_{1}$

$p_{2}$

$p_{3}$
Temperature

Fundamental frequency

Transformed source pressure amplitude

Sound pressure in the Cartesian coordinates

Source pressure amplitude

dimensionless $z$ axis

Source size in the $x$ direction

Source size in the $x$ direction

angular frequency

transformed transverse coordinate

transformed transverse coordinate

Focusing gain

Rayleigh distance

Small signal speed of sound

Small signal medium density

Transformed retarded time

Retarded time

Nonlinear parameter

Plane wave shock formation distance

Source temporal excitation

Source spatial aperture function

Source apodization function

Directional focusing gain in $x$ direction

Directional focusing gain in $y$ direction

Number of cycles

Nonlinearity of medium

Gol'dberg number

Pressure amplitude of the fundamental frequency

Pressure amplitude of the second harmonic

Pressure amplitude of the third harmonic 


\section{Chapter 4}

$T$

$p_{1}$

$p_{2}$

$p_{3}$

E

$E_{1}$

$E_{2}$

$E_{3}$

$\mathrm{B} / \mathrm{A}$

$\beta$

$c_{0}$

$\rho_{0}$

$\tau$

\section{Chapter 5}

T

$c_{0}$

$\lambda$

$\alpha$

$p_{1}$

$p_{2}$

$p_{3}$

$B E_{1}$

$B E_{2}$

$B E_{3}$

$C B E_{1}$

$C B E_{2}$

$C B E_{3}$

$\tau$
Temperature

Pressure amplitude of the fundamental frequency

Pressure amplitude of the second harmonic

Pressure amplitude of the third harmonic

Backscattered energy of the RF signal

Backscattered energy of the fundamental frequency

Backscattered energy of the second harmonic

Backscattered energy of the third harmonic

Parameter of nonlinearity

Coefficient of nonlinearity

Speed of sound

Medium density

Period

\section{Temperature}

Speed of sound

Thermal coefficient of sound speed

Linear coefficient of thermal expansion of the medium

Pressure amplitude of the fundamental frequency

Pressure amplitude of the second harmonic

Pressure amplitude of the third harmonic

Backscattered energy of the fundamental frequency

Backscattered energy of the second harmonic

Backscattered energy of the third harmonic

Change in backscattered energy of the fundamental frequency

Change in backscattered energy of the second harmonic

Change in backscattered energy of the third harmonic

Period 


\section{Chapter 1: Introduction}

\subsection{Heat and its Applications in Medicine and Biology}

Heat can destroy cancer cells by itself or sensitize them to radiation and chemotherapeutic drugs. Hyperthermia is a type of cancer treatment in which the tissue temperature is increased to $40-45^{\circ} \mathrm{C}$. Another type of thermal therapy is thermal ablation which directly kills cells and causes coagulation necrosis in tissue by elevating the temperature to above $55^{\circ} \mathrm{C}$. Hyperthermia and thermal ablation are the two types of cancer thermal treatments which are clinically approved $[1,2,3]$.

The most beneficial contribution of hyperthermia in oncology is based on enhancing the effectiveness of other treatment modalities such as radiation therapy and chemotherapy. Heat deposition in the hyperthermia regime has minimal damage to tissues but it increases their radiosensitivity by a factor between 1.2 to 5 [4]. Poor tissue oxygenation causes transient or chronic hypoxia and cells in hypoxic regions are relatively radioresistant. Local hyperthermia can increase blood flow in tumor region which improves tissue oxygenation leading to an increase in radiosensitivity [4].

Drug delivery to tumor tissue would be improved at the region of hyperthermia due to several mechanisms. Heat increases blood flow and perfusion that increases the intravascular pressure. It also increases the pore sizes between endothelial junctions in tumor tissues. The combination of these two effects increases drug extravasation from the vascular space to the cancerous tissue creating high local concentrations of drug within the tumor [5]. It has also been

reported that hyperthermia can increase the drug cellular uptake due to increased cellular 
permeability. Therefore, cancer treatment could be enhanced by combining hyperthermia with chemotherapy or radiotherapy [5].

Hyperthermia has also been combined with small-molecule drugs, long-circulating nanoparticle drug carriers and thermosensitive liposomes (TSL) in order to improve cytotoxicity of the drugs [5]. A liposome is an artificial vesicle composed of a lipid bilayer boundary which can be used to deliver pharmaceutical drugs to tumor tissues [6]. The drug inside a liposome can be released by several chemical and physical stimuli such as heat, $\mathrm{pH}$ level and light [6]. Thermosensitive liposomes are being developed in such a way as to release the drug within the hyperthermia temperature range $\left(39-43^{\circ} \mathrm{C}\right)$, but to remain stable in non-tumor bloodstream at physiological temperature $\left(37^{\circ} \mathrm{C}\right)$ (Figure 1-1). This reduces the exposure of healthy organs to chemotherapy and lowers systemic toxicity [7].
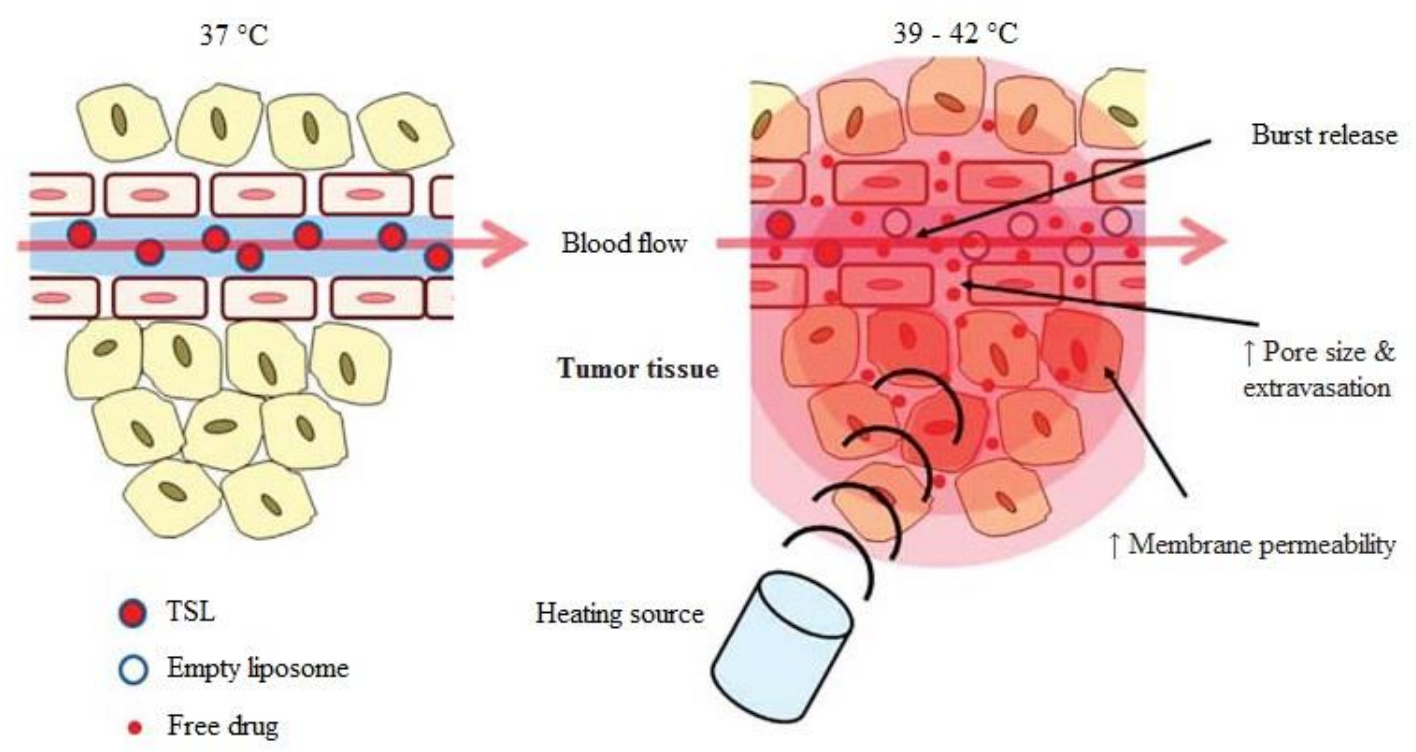

Figure 1-1: Effect of ultrasound hyperthermia on TSL uptake at tumor region [5].

It is well stablished that the cellular damage caused by heat is a function of both temperature and time. Both in vivo and in vitro experiments indicate that there is an exponential relationship between temperature and exposure time. As stated by Sapareto and Dewey [8], a one 
degree increase in temperature requires a two-fold decrease in time for the same biological response above $43^{\circ} \mathrm{C}$ and a three-to-four fold decrease in time for an iso-effect below $43^{\circ} \mathrm{C}$. The concept of thermal dose was introduced in order to quantitatively describe the cumulative effect of temperature on cells and tissues over time. The thermal iso-effect dose delivered to tissue is defined as the cumulative equivalent minutes at $43^{\circ} \mathrm{C}, t_{43}$, according to [8]:

$$
t_{43}=\sum_{t=0}^{t=t_{\text {final }}} R^{(43-\overline{T(t)})} \Delta t
$$

where $t$ is the heating exposure time and $\overline{T(t)}$ is the average temperature during desired interval of heating $(\Delta t) . R$ is a constant that equals to 0.5 for temperatures above $43^{\circ} \mathrm{C}$ and 0.25 for temperatures below $43^{\circ} \mathrm{C}$.

As mentioned above, there are two general approaches to thermal therapy: hyperthermia and thermal ablation. In general, the former has a longer treatment time than the latter and is used in combination with radiation therapy and/or chemotherapy. In the thermal ablation approach, the heating device rapidly coagulates the tumor by itself, and therefore, normally, no other modality is used in conjunction with this treatment [9]. However, ablating a large volume, irregularly-shaped or highly-perfused tumors is a challenge. Similar to hyperthermia, thermal ablation could be combined with thermosensitive and non-thermosensitive liposomes. As shown in Figure 1-2, the core of the tumor could be coagulated by ablation $\left(\mathrm{T}>55^{\circ} \mathrm{C}\right)$ while the surrounding tissue, with lower temperatures $\left(\sim 42^{\circ} \mathrm{C}\right)$, become more susceptible to chemotherapy agents [5]. 


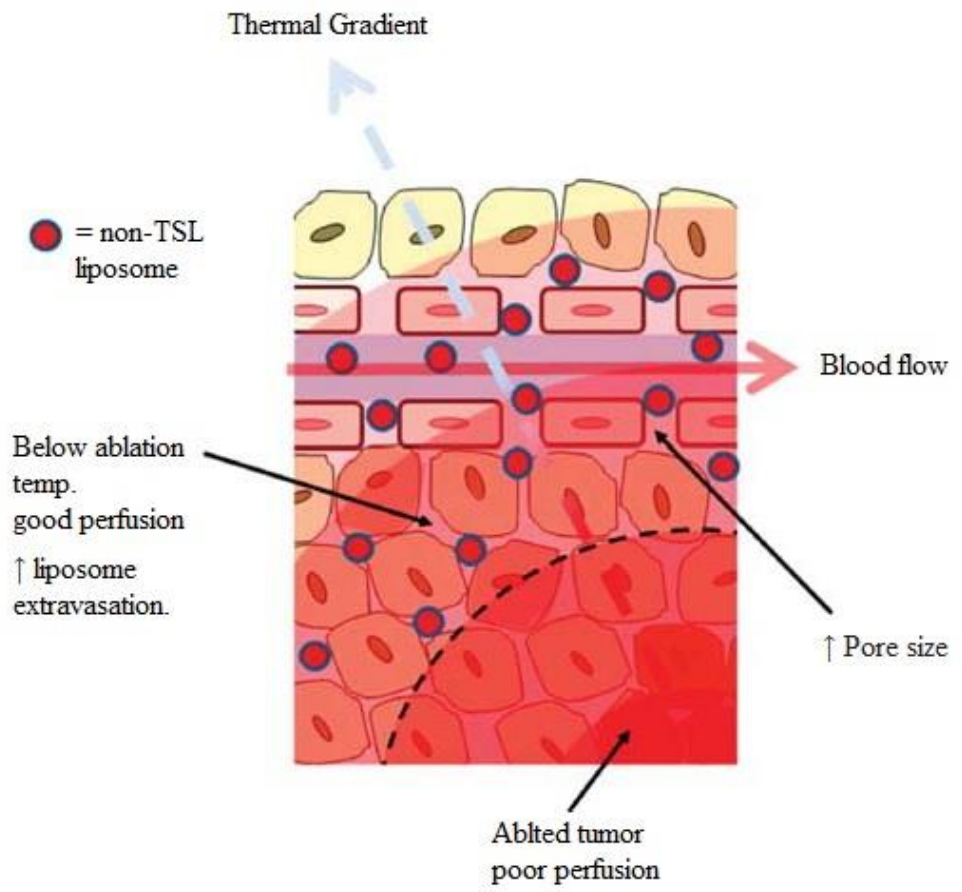

Figure 1-2: Effect of thermal ablation therapy on non-TSL uptake at tumor region. Adapted from [5].

\subsection{Tissue Heating Modalities}

There are a number of heating methods available for tissue thermal therapy such as ultrasound, interstitial microwave, radio frequency and laser that will be briefly described.

\subsubsection{Laser Thermotherapy}

Laser therapy is suitable for skin tumors and superficial cosmetic surgeries due to the fact that laser is highly scattered in tissue which decreases its penetration depth significantly [10]. Therefore, in cases of deeply embedded cancerous tumors, minimally invasive laser-induced interstitial thermotherapy (LITT) using fiber optic catheters are performed which deliver the laser energy into the center of the tumor. Laser fibers are thin and can be placed within the tumor with minimal damage to surrounding tissues [11].

The main thermal effects that may occur during laser heating are: 1) carbonization which decreases the penetration depth of laser photons due to increasing the tissue absorption [12], 2) 
vaporization which causes explosive tissue rupture and 3) overheating of the applicator that may damage the device [13].The disadvantage of laser is that it needs a very high range of power levels (hundreds of watts) to coagulate tissue and also it is not selective and damage both normal and cancerous tissues along its path [14]. Tissue perfusion plays a significant role in laser heating process and it is one of the parameters indicating the sharpness and the power of laser beam [14, $12]$.

\subsubsection{Gold Nanoparticle Thermotherapy}

Many studies have been performed in recent years on applying laser energy to embedded gold nanoparticles (GNP) in order to heat localized regions of body [15]. This photothermal therapy is based on low absorption of soft tissue within the wavelength of laser ranging from 600 to $1400 \mathrm{~nm}$ which has provided a therapeutic window due to its low absorption in this range [16].

Gold nanoparticle thermotherapy provides a precise localized area of heating. Targeted GNPs can be placed in the region of interest where the treatment is intended to be performed [17]. GNPs can significantly increase the temperature when exposed to laser radiation near their plasmon-resonant absorption band. A temperature increase of up to $12^{\circ} \mathrm{C}$ can be induced by using a relatively low power intensity laser $\left(1\right.$ to $\left.5 \mathrm{~W} / \mathrm{cm}^{2}\right)$ in few minutes which protects surrounding tissues [18].

GNPs can be produced in different shapes which each has unique properties that make them to be suitable for different kinds of cancerous tumor in various parts of the body. However, GNPs have relatively high production cost and since it is a recent technology, a lot of work has to be done in order to be successfully adopted in clinical use [19]. 


\subsubsection{Microwave Thermotherapy}

Minimally-invasive interstitial microwave (MW) antennas can be placed in tumors deep within the body to induce large thermal lesion formation (relative to laser thermotherapy). MW sources can be used as a single antenna or as an array of antennas [20]. The phase relationship between antennas enables field overlap which leads to larger ablation zones. Development in design of the MW antennas has enhanced the heating procedure in different parts of human body such as lung, kidney, bone, chest wall, liver and brain. MW antenna cooling has been accomplished with circulating water/saline, or flowing air which enables longer duration of treatment to achieve larger area of coagulation with more spherical volume [20].

Conformal microwave array applicator is also used for large area chest wall disease which is the largest superficial hyperthermia applicator [21]. However, MW has a long treatment time and it cannot be focused. Skin burn is one of the other disadvantages of this method which can be minimized by using water bolus [21].

\subsubsection{Radio-frequency Thermotherapy}

Radio-frequency (RF) heating is one of the most commonly used modalities for thermal therapy. RF heating, same as microwave, can be applied to both thermal therapy methods: mild hyperthermia and thermal ablation. However, these methods are quite different in mechanism of heating and therefore they have different limitations. RF heating can be applied by monopolar or bipolar modes in the frequency range of 10-120 MHz. In monopolar mode, a single or a group of interstitial electrodes are used to deliver RF current whereas in bipolar mode, two parallelopposite surface electrodes are needed in order to apply the electrical current at the tumor region $[22,23,24]$. 
RF heating is dependent on electric and thermal conductivity of the tissue to allow current flow and thermal diffusion, respectively. High conductive tissues are the ones with high percentage of water in which RF energy can effectively penetrate. On the other hand, RF energy can hardly penetrate in organs with low conductivity such as lung and bone $[25,26]$.

The advantage of microwaves over RF is that microwaves can propagate through low or zero electric conductive tissues whereas RF heating requires an electrically conductive path. High conductive tissues are the main absorber of microwave energy while low conductive tissues allow the microwave to propagate [25].

Short-wave diathermy (SWD) is a form of radiofrequency radiation to induce thermal effects in the body [27]. Since the SWD is an electromagnetic radiation, it is composed of an electric field and a magnetic field which either one can be used for treatment. The one using the electric field is known as the capacitive method and the one using the magnetic field is known as the inductive method [28]. In the capacitive method, the electrostatic field is induced in the region of interest by placing it between two electrodes. The inductive method is applied with only one electrode. SWD can be applied in either continuous shortwave diathermy (CSWD) or pulsed short-wave diathermy (PSWD) mode. However, the continuous shortwave diathermy is the one typically used for thermal treatment [27, 28].

\subsubsection{Ultrasound Thermotherapy}

Ultrasound has many advantages in comparison to other heating modalities due to its high penetration depth in soft tissues and high degree of beam forming control. Moreover, ultrasound energy can be applied non-invasively to tissue. However, there is a tradeoff between the frequency and the penetration depth of an ultrasound beam. Ultrasound can be used to 
increase the body temperature to hyperthermia temperature range $\left(42\right.$ to $\left.45^{\circ} \mathrm{C}\right)$ or to higher than $55^{\circ} \mathrm{C}$ in order to thermally ablate (coagulate) tumors $[29,30,31]$.

Two types of clinical ultrasound devices currently available are extracorporeal and transrectal [32]. The former has a longer focal length $(10$ to $16 \mathrm{~cm})$ to heat target regions within abdomen and the latter, which has a shorter focal length $(3$ to $4 \mathrm{~cm})$ is used mostly for prostate cancer $[29,30,32]$. Ultrasound is also a non-ionizing source which allows the treatment to be repeatable [29].

There are two major limitations that apply to therapeutic ultrasound beams when they transmit into the body. Complete reflection of the beam at tissue-air interfaces causes treatment limitations in lungs and regions of body which contain gas. Also, the gaseous regions reflect the beam towards the transducer and may cause severe injury in tissues between the gaseous region and the transducer due to the high energy of the beam. High beam absorption in bone and the partial reflection of the beam at tissue-bone interfaces cause high temperature and pain in the patient [33]. Cavitation bubble formation is an important non-thermal effect during HIFU which results in relatively unpredictable lesions [34].

\subsection{Invasive Tissue Thermometry}

Invasive clinical thermometry is primarily performed using thermocouples or fibre-optic sensors [35]. However, these devices are invasive and give only point temperature measurements in tissue [36, 37]. The presence of the sensor may also modulate the thermal therapy field [38]. Due to these reasons, reliable noninvasive tissue thermometry methods are highly preferable especially in clinical applications. 


\subsection{Noninvasive Tissue Thermometry}

Ultrasound hyperthermia can be used to produce a well localized heating with a deep penetration within the body. However, tissue heterogeneity and perfusion cause non-uniformities in heating especially in the tumor tissue [3]. The other major limitation of ultrasound hyperthermia is the lack of a reliable noninvasive and real time method of temperature monitoring and control to guide the heating during therapy. Monitoring the temperature distribution during the therapy helps to maintain the target temperature at the region of interest and to increase the effectiveness and safety of the treatment. "Successful implementation of real-time noninvasive temperature estimation will be a boon for thermal therapy as it becomes less invasive and as the heating sources become more sophisticated" [39].

Therapeutic TSLs are designed in a way that hyperthermia triggers the release of drugs at temperatures around $40-42^{\circ} \mathrm{C}$. This is a narrow temperature range for triggering the drug release and increasing the blood perfusion since vascular termination may occur above $43^{\circ} \mathrm{C}$ [7]. Therefore, accurate control over the temperature is an important aspect of this procedure. Without a precise temperature control during the hyperthermia treatment which may take over 30-60 minutes, the function of these thermo-sensitive therapeutic agents will be significantly hampered. Different noninvasive modalities including magnetic resonance imaging (MRI), ultrasound and photoacoustics (PA) thermometries have been proposed to this end [40, 41, 42].

\subsubsection{MR Thermometry}

Currently, MR thermometry is considered as the gold standard modality for noninvasive tissue temperature measurements in clinics [40]. An excellent review on different MR thermometry techniques for monitoring thermal therapy is published by Rieke and Butts Pauly 
[40]. There are several MR parameters that make MRI suitable for measuring absolute temperature, temperature change and tissue thermal damage (ablation) during clinical thermal treatments. Proton density, $\mathrm{T}_{1}$ and $\mathrm{T}_{2}$ relaxation times, the diffusion coefficient and proton resonance frequency (PRF) shift are some of the MR parameters that are sensitive to temperature [40]. The PRF shift-based method is the most utilized technique for clinical use in terms of temporal resolution, linearity of the signal over a large temperature range (from $-15^{\circ} \mathrm{C}$ to $100^{\circ} \mathrm{C}$ ) and sensitivity. The PRF thermal coefficient is nearly independent of tissue type (except for fat a.k.a. adipose tissue) and tissue coagulation [40].

The resonance frequency $\left(\omega^{\prime}\right)$ of a proton in a molecule is determined by the product of the gyromagnetic ratio $\left(\gamma^{\prime}\right)$ and the local magnetic field $\left(B_{l o c}\right)$ shown as:

$$
\omega^{\prime}=\gamma^{\prime} B_{l o c},
$$

where the local magnetic field can be written as:

$$
B_{l o c}=\left(1-s^{\prime}\right) B_{0}
$$

where $s^{\prime}$ is called the screening (or shielding) constant and $B_{0}$ is the magnetic flux density. As the temperature increases, hydrogen bonds of water molecules stretch, bend, and break, which increases the electron screening effect [40]. An increase in the screening effect decreases the local magnetic field. Therefore, the PRF decreases due to the decrease in local magnetic field. The average electron-screening constant of water decreases linearly with temperature at a ratio of $-1.03 \pm 0.02 \times 10^{-8} /{ }^{\circ} \mathrm{C}[40]$.

Image-guided thermal therapy is clinically being performed with magnetic resonance imaging-guided focused ultrasound (MRgFUS). MRgFUS combines the anatomical imaging and 3-D temperature monitoring capabilities of MRI and the therapeutic abilities of focused ultrasound (FUS). Currently, MRgFUS is clinically applied for the non-invasive ablation of 
uterine fibroids. Current investigations focus on determining the clinical feasibility of MRgFUS in the treatment of benign and malignant tumors of the breast, prostate, liver, pancreas, and uterus $[43,44]$. However, MRI is an expensive imaging device with high maintenance costs; it is not portable and the heating applicator has to be MR compatible [40, 43]. Due to these complications, there is a great interest in research and clinical communities towards less complicated non-MRI thermometry methods suitable for clinical applications.

\subsubsection{Photoacoustic thermometry}

Photoacoustic thermometry is another temperature measurement method which is an emerging technique and is still in its infancy $[45,46,47]$. The photoacoustic (PA) effect is based on absorption of light energy by an object. Some of the absorbed photon energy converts into heat and the object temperature increases. The increase in temperature leads to rapid thermoelastic expansion, generating acoustic pressure in the medium which can be detected by an ultrasound transducer. A short laser pulse is usually used to irradiate the tissue in order to satisfy the thermal and the acoustic stress confinement conditions [48].

The photoacoustic signal amplitude has been reported to increase linearly as a function of temperature with a relatively high sensitivity [42]. The PA signal amplitude is proportional to the local fluence of laser and to the Grüneisen parameter, $\Gamma^{\prime}$, which is defined as [42]:

$$
\Gamma^{\prime}=\frac{\alpha_{V} c_{0}^{2}}{C_{p}},
$$

where $\alpha_{V}\left[{ }^{\circ} \mathrm{C}^{-1}\right]$ is the thermal volume expansion of the medium, $c_{0}[\mathrm{~m} / \mathrm{s}]$ is the speed of sound and $C_{p}\left[\mathrm{~J} \mathrm{~g}^{-1}{ }^{\circ} \mathrm{C}^{-1}\right]$ is the specific heat capacity at a constant pressure. Inspection of Eq. 1-4 shows that the Grüneisen parameter is proportional to the thermal volume expansion and the squared speed of sound. The local fluence is proportional to the optical attenuation coefficient which is 
not significantly dependent on temperature. Therefore, PA thermometry relies on the dependency of the Grüneisen parameter on temperature. Pramanik et al. showed that the PA signal obtained from a hot ink sample, which was allowed to cool down to room temperature, followed the actual temperature with an accuracy of $\pm 0.6^{\circ} \mathrm{C}$ [49]. Larina et al. performed PA temperature measurements in ex vivo canine liver, canine myocardium and gelatin phantom [42]. By increasing the temperature from $37^{\circ} \mathrm{C}$ to $43^{\circ} \mathrm{C}$, approximately $9 \%$ and $4 \%$ increase was observed in the pressure amplitude in liver and myocardium tissues, respectively. Photoacoustic temperature monitoring in the liver tissue and the gel phantom was done with an accuracy of less than $1^{\circ} \mathrm{C}$ at depths of up to $3 \mathrm{~cm}$ from the transducer surface [42].

\subsubsection{Ultrasonic thermometry}

Ultrasound is a non-ionizing, inexpensive and portable modality which could potentially be a superior alternative to other types of thermometry systems. Lewis et al. have extensively reviewed various ultrasound thermometry techniques [38].

Previously, three major ultrasonic methods have been proposed to measure the tissue temperature non-invasively. They are based on estimations of: 1) the variation in the attenuation coefficient, 2) the change in backscattered signal energy (CBE), and 3) the backscattered RF echo-shift or the apparent displacements of the ultrasound speckle due to the change in the speed of sound (SOS) and thermal expansion of the medium [41,38]. Studies have also shown that the acoustic nonlinearity parameter (B/A) and the acoustic harmonic amplitude ratios are

temperature dependent and could be used for ultrasound thermometry [50, 51, 52, 53]. However, to date, none of these techniques have been clinically implemented. 


\subsubsection{Tissue Thermometry based on Change in Attenuation Coefficient}

There are two phenomena that change the shape of an ultrasound waveform as it propagates in a medium: frequency-dependant attenuation and the nonlinearity of the medium [54]. The first effect results in the ultrasound wave losing energy as it propagates - the wave amplitude drops. This dissipation is due to both effects of absorption and scattering of the wave along its path and it can be mathematically expressed as [54]:

$$
\bar{I}(x)=\bar{I}(0) e^{-2\left(\alpha_{s}+\alpha_{a}\right) x}=\bar{I}(0) e^{-2 \alpha x},
$$

where $\bar{I}(0)$ is the averaged incident intensity at $x=0, \bar{I}(x)$ is the average intensity at a location $x, \alpha_{s}$ and $\alpha_{a}$ are the scattering and absorption amplitude attenuation coefficients, respectively. The sum of $\alpha_{s}$ and $\alpha_{a}$ has been defined as the amplitude attenuation coefficient, $\alpha$ which is mainly dependent on frequency and temperature. The frequency dependence of attenuation coefficient for most soft tissue is modeled as:

$$
\alpha(f)=\alpha_{0} f^{n}
$$

where $\alpha_{0}$ is the attenuation coefficient at $1 \mathrm{MHz}$ and $n$ represents the frequency dependence of the attenuation coefficient which lies in the range from 1 to 2 for soft tissues [54]. This implies that higher frequencies have higher attenuation and therefore the center frequency of the wave will be down-shifted as it propagates in an attenuative medium [54].

In case of pure fluids there is no scattering, and therefore, the attenuation is only due to absorption. Stokes obtained the classical equation for the absorption of sound in fluids $\left(\alpha_{c}\right)$ by assuming that the propagation process for a compressional wave to be adiabatic and ignoring the effects of bulk viscosity which can be represented as [54]: 


$$
\alpha_{c} \approx \frac{2 \omega^{2} \kappa \eta_{s}}{3 c_{0}}
$$

where $\kappa$ is the adiabatic compressibility, $\eta_{s}$ is the shear viscosity and $\omega$ is the angular frequency. The shear viscosity arises from velocity differences between adjacent liquid layers. Subsequently, Kirchoff added a thermal conduction term to the absorption coefficient that can be written as [54]:

$$
\alpha_{c}=\frac{\omega^{2} \kappa}{2 c_{0}}\left[\frac{4}{3} \eta_{s}+(\gamma-1) \frac{\mathrm{\kappa}}{C_{p}}\right] \text {, }
$$

where $\gamma=C p / C v$ is the ratio of specific heats and $K$ is the thermal conductivity of the medium. The value of specific heat ratio for most liquids is very close to 1 due to low compressibility and as a result the thermal conduction term is negligible for liquids [54]. However, the calculated values of the classical absorption coefficient were much smaller than the measured values which is due to ignoring other loss mechanisms in the equation. To account for other loss mechanisms such as thermal and structural relaxation, an additional bulk (volume) viscous term was added. Adding the bulk viscosity $\left(\eta_{b}\right)$ gives correct values for the absorption confident in liquids. Therefore, the theoretical absorption coefficient in liquids, $\alpha_{t}$, could be given by:

$$
\alpha_{t}=\frac{\omega^{2} \kappa}{2 c_{0}}\left[\frac{4}{3} \eta_{s}+\eta_{b}+(\gamma-1) \frac{\kappa}{C_{p}}\right] .
$$

It has been shown that in liquids where shear viscosity is the dominant mechanism (or in the case of any other dominant high-frequency structural relaxation where the frequency dependence of the attenuation coefficient is 2 as in water), $\alpha$ is a monotonically negative function of temperature and follows approximately the same temperature dependence as the shear viscosity [55]. 
Several groups have investigated the effect of temperature on attenuation coefficient in soft tissues at above and below $50^{\circ} \mathrm{C}[56,57,58,59,55]$. However, studies have shown various results of the temperature dependence of attenuation measurements in soft tissues below 40$50^{\circ} \mathrm{C}$. As stated by Bamber, "no single absorption or scattering mechanism has yet been used to explain the observed temperature dependences of attenuation in soft tissues" [55]. However, most studies show that at temperatures below $40-50^{\circ} \mathrm{C}$ and frequencies above $2 \mathrm{MHz}$, the attenuation coefficient in soft tissue decreases with temperature with a slope $(d \alpha / d T)$ as a decreasing function of frequency and temperature [55]. When tissue temperature goes above 40$50^{\circ} \mathrm{C}$ (or the thermal dose exceeds $240 \mathrm{~min}$ at $43^{\circ} \mathrm{C}$ ), coagulation necrosis occurs which is a permanent structural change in the tissue. Attenuation coefficient increases irreversibly when tissue undergoes coagulation. However, at lower temperatures (hyperthermia temperature range), the changes of attenuation are reversible [55].

Jackson et al. showed that the attenuation in ex vivo bovine liver slightly decreased with heating for temperatures lower than $50^{\circ} \mathrm{C}$ and then increased after $50^{\circ} \mathrm{C}$ (from $6.48 \pm 0.02 \mathrm{Npm}^{-1}$ at $23^{\circ} \mathrm{C}$ to $5.2 \pm 0.37 \mathrm{Npm}^{-1}$ at $50^{\circ} \mathrm{C}$ ) at $1 \mathrm{MHz}$ [51].

Choi et al. measured the attenuation coefficient in ex vivo porcine liver tissue at the frequencies of $1,2,3,4$ and $5 \mathrm{MHz}$ as a function of temperature from $30^{\circ} \mathrm{C}$ to $75^{\circ} \mathrm{C}$. The attenuation coefficient decreased with temperature at all the frequencies and reached its minimum at about $50^{\circ} \mathrm{C}[50]$.

Ghoshal et al. showed that the attenuation coefficient decreased by $10 \%-20 \%$ with temperature from $37^{\circ} \mathrm{C}$ to $50^{\circ} \mathrm{C}$ in rabbit and beef liver [60]. Some studies have shown that the slope of the decrease in attenuation with temperature (up to $50^{\circ} \mathrm{C}$ ) is steeper at higher frequencies. Bamber and Hill studied the temperature dependence of the attenuation coefficient 
in fixed bovine brain, spleen and liver and freshly excised bovine liver, peritoneal fat and human liver over the frequency range of 1 to $7 \mathrm{MHz}$ for the temperature range of 5-65 ${ }^{\circ} \mathrm{C}$ [58]. They observed that attenuation is strongly decreasing with temperature at higher frequencies but the temperature coefficient of attenuation decreases with the reduction in frequency. Gammell et al. measured the attenuation coefficient in porcine liver, kidney, back fat and spleen and human liver at temperatures 4,20 and $37^{\circ} \mathrm{C}$ and frequencies from 1.5 to $10 \mathrm{MHz}$ [61]. The attenuation coefficient in all the samples showed a decreasing trend as a function of temperature with a higher sensitivity at higher frequencies.

Attenuation coefficient in canine liver tissue was measured by Techavipoo et al. from $22^{\circ} \mathrm{C}$ to $90^{\circ} \mathrm{C}$ at three center frequencies [56]. The attenuation coefficient decreased by $-19 \%,-$ $21 \%$ and $-28 \%$ at frequencies of 4,5 and $6 \mathrm{MHz}$, respectively as the temperature was elevated from $22^{\circ} \mathrm{C}$ to $50^{\circ} \mathrm{C}$.

On the other hand some studies concluded that attenuation is highly sensitive to temperature at temperatures above $50^{\circ} \mathrm{C}[56,57,58,59]$. Damianou et al. demonstrated the temperature dependence of attenuation coefficient in dog muscle, kidney and liver for temperatures from $20^{\circ} \mathrm{C}$ up to $70^{\circ} \mathrm{C}$ at $4.32 \mathrm{MHz}$ (Figure 1-3) [57]. They showed that the change in attenuation with temperature is insignificant for temperatures up to $50^{\circ} \mathrm{C}$, and then increases rapidly until it reaches $65^{\circ} \mathrm{C}$. 


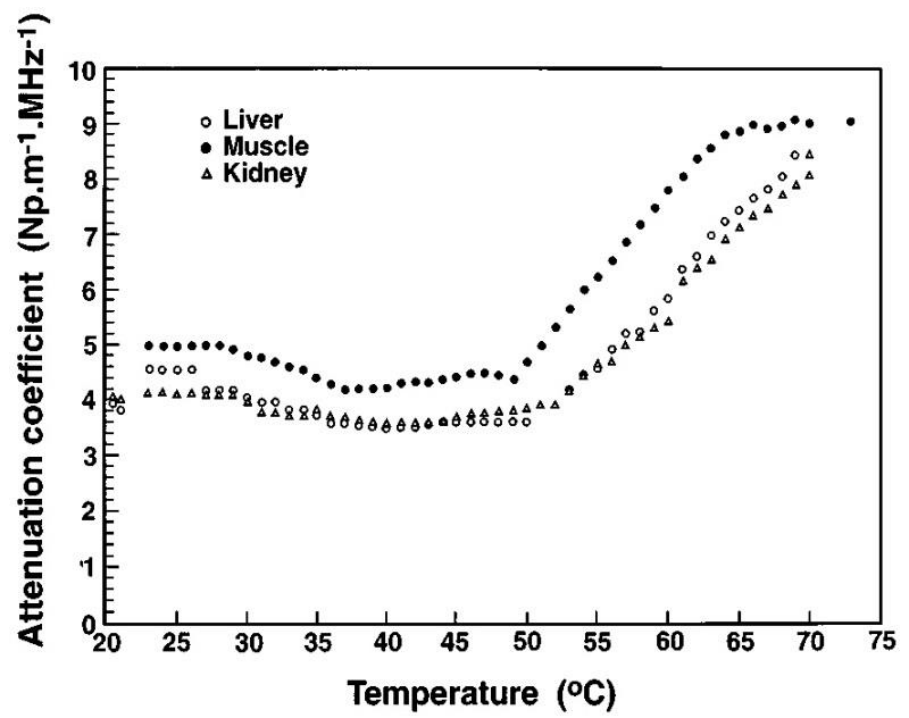

Figure 1-3: Attenuation coefficient versus temperature for dog muscle, liver, and kidney in vitro [57].

Parmar and Kolios showed that the attenuation of porcine liver increases significantly at $45^{\circ} \mathrm{C}$ and greater [59]. Therefore, measurement of change in ultrasonic attenuation can be a promising method for monitoring thermal ablation therapy were the tissue temperature is elevated above $50^{\circ} \mathrm{C}$.

\subsubsection{Tissue Thermometry based on Change in Backscattered Energy}

The change in the ultrasound backscattered energy $(\mathrm{CBE})$ is another parameter that varies as a function of temperature. This can be measured by the examination of the change in the amplitude of the RF echo signal (signal strength). Arthur et al. have stated that in an ex vivo bovine liver sample and in an in vivo animal study, the backscattered energy changes monotonically with temperature with either positive or negative slope whether the tissue is made of aqueous or lipid scatterers, respectively $[62,63]$. This interpretation was based on the theoretical model of the backscattered power from a small tissue volume containing a random distribution of scatterers [64]. However, backscattered energy depends on the number, spacing, density and the material of the scatterers, which are complicated to identify due to tissue 
inhomogeneity within the human body [54]. Vlad et al. have shown that cell death is another parameter which changes the backscattered energy [65].

Li et al. showed that some mechanisms other than change in scattering cross section may also cause change in backscattered energy [66]. Changes in speckle pattern could also cause CBE. The changes in the speckle pattern can be due to at least two factors: (1) change in speed of sound with temperature which changes the wavelength and the time of flight, and (2) shifting of the scanning plane between acquisitions of image frames [66].

\subsubsection{Tissue Thermometry based on Echo-Shift Technique}

The backscattered RF echo-shift technique, which has been shown to be the most promising ultrasound-based thermometry method, is due to two physical phenomena: 1) the change in speed of sound with temperature and 2) the medium thermal expansion due to heating [67]. The former causes an apparent and the latter causes an actual motion in the position of scatterers. Apparent shift in scatterer position is due to the assumption of a fixed SOS by the imaging systems. However, the change in SOS dominates the thermal expansion in the hyperthermia temperature range $\left(37^{\circ} \mathrm{C}-45^{\circ} \mathrm{C}\right)$ [68]. The speed of sound for a small-amplitude acoustic signal is related to the adiabatic compressibility given by:

$$
c_{0}=\sqrt{\frac{1}{\kappa \rho_{0}}}
$$

where $\rho_{0}$ is the density of the medium at equilibrium. Figure 1-4 shows the temperature dependence of the adiabatic compressibility and the speed of sound in water. The speed of sound is increased initially as the temperature rises and reaches a maximum value of $1557 \mathrm{~m} / \mathrm{s}$ at $74^{\circ} \mathrm{C}$ 
and then decreases. This is mainly due to the temperature dependence of $\kappa$ which decreases to a minimum at approximately $66^{\circ} \mathrm{C}$ and then increases [54].
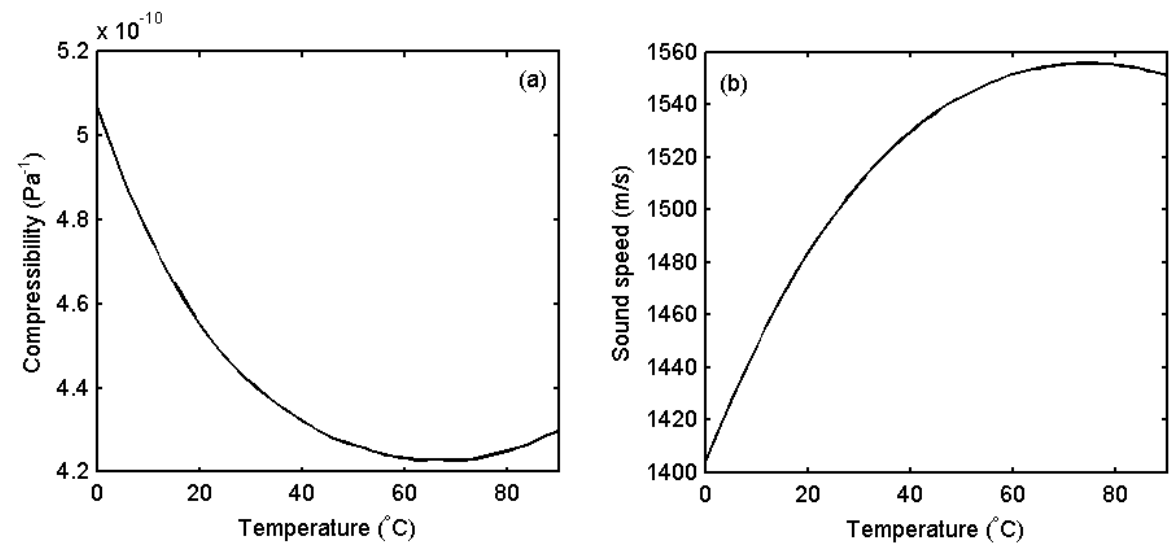

Figure 1-4: (a) The adiabatic compressibility and (b) the speed of sound in water as a function of temperature [54].

In 1998, Ebbini's group presented the relation between the change in temperature and the axial time-shift in the ultrasound RF signal [67]. In this method, temperature change measurement is accomplished by obtaining the echo-shifts in the RF echo signals at each location of the medium, differentiating them along the axial direction and scaling them by a tissue dependent constant. Assuming that the SOS is linearly dependent to temperature in tissue from the normal body temperature to $45^{\circ} \mathrm{C}\left(\sim 10^{\circ} \mathrm{C}\right.$ temperature change $)$ and the SOS is constant throughout the sample, the temperature change could be written as [67]:

$$
\delta T(z)=\frac{c_{0}}{2}\left(\frac{1}{\alpha_{L}-\lambda}\right) \frac{d}{d z}(\delta t(z))
$$

where $\lambda\left[{ }^{\circ} \mathrm{C}^{-1}\right]$ is the thermal coefficient of the SOS, $\alpha_{L}\left[{ }^{\circ} \mathrm{C}^{-1}\right]$ is the linear coefficient of thermal expansion of the medium, and $\delta t(z)$ is the cumulative time-shift at depth $z$. The term $K=$ $\frac{c_{0}}{2}\left(\frac{1}{\alpha_{L}-\lambda}\right)$ is a material dependent factor which accounts for both the thermal dependence of the SOS and thermal expansion of the medium which can be experimentally determined [67]. The 
incremental time-shift map $\left(\delta t(z)_{\text {incr }}\right)$ between two successive RF frames at times $t_{1}-t_{2}, t_{2}-t_{3}, \ldots$, $t_{\mathrm{i}}-t_{\mathrm{i}+1}$ with temperatures of $T_{1}-T_{2}, T_{2}-T_{3}, \ldots, T_{\mathrm{i}}-T_{\mathrm{i}+1}$ are first acquired by using a classical cross correlation technique at each location $(z, x)$. The cumulative time-shift $\delta t(z)$ is obtained by summing all the incremental time-shifts. Differentiating the cumulative time-shift map along axial direction yields the gradient or strain map. The temperature change map will be estimated by scaling the gradient map by the $K$ value.

The temperature coefficient of the speed of sound, $\lambda$, for water-based tissue is positive and it is in the range of $0.7 \times 10^{-3}$ to $1.3 \times 10^{-3}{ }^{\circ} \mathrm{C}^{-1}$. For lipid-based tissue this coefficient is negative and ranges from $-1.3 \times 10^{-3}$ to $-2 \times 10^{-3}{ }^{\circ} \mathrm{C}^{-1}$. Some studies ignore the contribution of thermal expansion due to the fact that around $37^{\circ} \mathrm{C}, \lambda$ overwhelms $\alpha_{L}$ by at least an order of magnitude. However, above $50{ }^{\circ} \mathrm{C}$ this assumption is not valid [69].

Unfortunately there are several challenges that face noninvasive thermometry based on the echo-shift technique. Robust temperature estimation has not been demonstrated in vivo and has not been applied clinically.

A major challenge for in vivo applications is related to tissue motion, which includes respiratory and cardiac motion. Tissue motion can mask the temporal shifts caused by the temperature change since these are typically an order of magnitude smaller than the tissue motion shifts. Tissue motion produces mechanical shifts which are equivalent to temporal shifts and appear as significant source of artifact in temperature maps.

The speed of sound in the medium is a function of temperature, therefore an ultrasonic beam propagating through a heated region with a non-uniform sound speed distribution gets distorted [70]. This phenomenon is called "thermo-acoustic lens" effect which presents as spatial 
ripple beyond the heated region and appears as an artifact in temperature maps obtained using the echo-shift technique.

\subsubsection{Tissue Thermometry based on Acoustic Harmonics}

As mentioned before, the second phenomenon that changes the shape of a waveform is the acoustic nonlinearity, which is due to the variation of the speed of sound $[54,71]$. The speed of sound for a small-amplitude signal has been assumed to be constant for all phases of the wave. However, this assumption is not true for a relatively high pressure wave, a.k.a. a finite-amplitude wave. For a finite-amplitude wave the speed is dependent on medium density at a given point (phase) in the wave and hence it is higher in compression phases and lower at rarefaction phases [54]. This variation in the phase velocity causes the wave to progressively get distorted as it propagates in the medium and to generate higher order harmonics as shown in Figure 1-5.
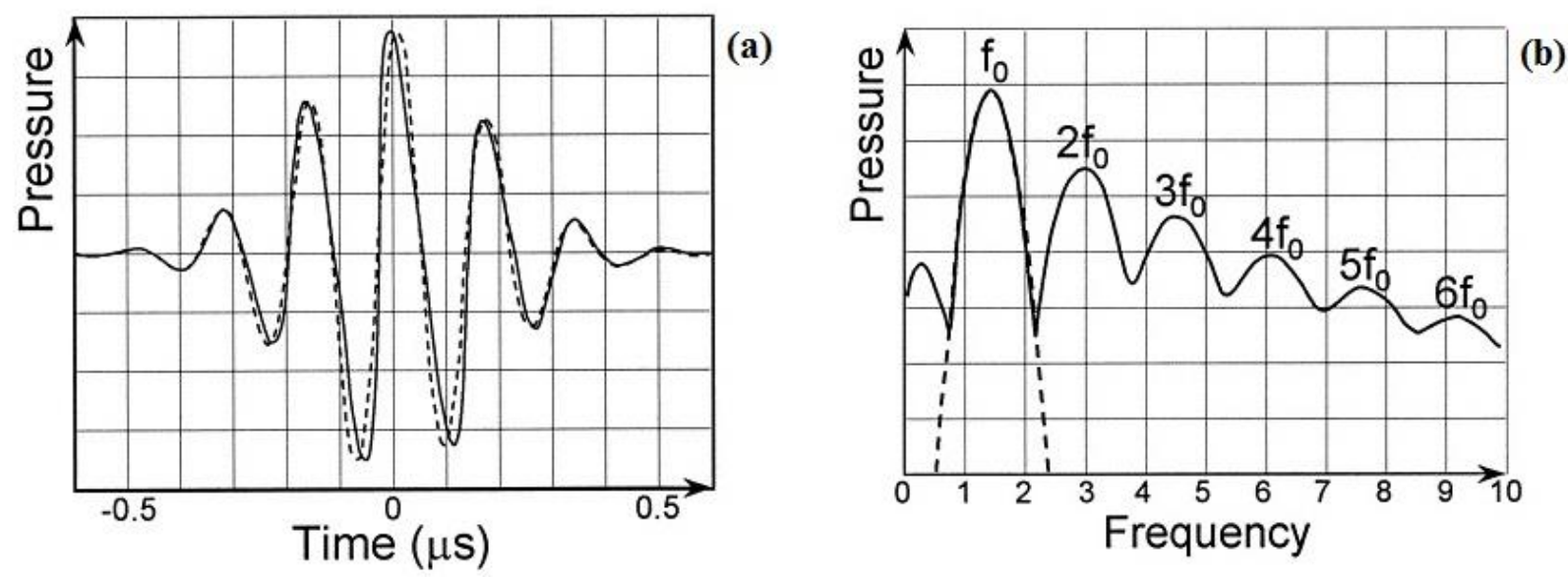

Figure 1-5: (a) The dotted line presents a pulse at $0 \mathrm{~cm}$ and the solid line presents the distorted pulse due to nonlinearity at $10 \mathrm{~cm}$ in water. (b) The dotted line represents the normalized frequency spectrum of the signal at 0 $\mathrm{cm}$ and the solid line represents the normalized frequency spectrum of the distorted pulse at $10 \mathrm{~cm}$ which has generated multiple harmonics [72].

The pressure-density relation for liquids can be written as an equation of state $p=$ $p(\rho, s)$, where $p=p_{0}+p_{1}$ and $\rho=\rho_{0}+\rho_{1}$. The subscripts 0 and 1 refer to equilibrium and 
perturbed values, respectively and $s$ is the entropy. The Taylor series expansion of the equation of state under adiabatic conditions yields [54]:

$$
p-p_{0}=\left(\frac{\partial p}{\partial \rho}\right)_{s, 0}\left(\rho-\rho_{0}\right)+\frac{1}{2 !}\left(\frac{\partial^{2} p}{\partial \rho^{2}}\right)_{s, 0}\left(\rho-\rho_{0}\right)^{2}+\frac{1}{3 !}\left(\frac{\partial^{3} p}{\partial \rho^{3}}\right)_{s, 0}\left(\rho-\rho_{0}\right)^{3}+\cdots
$$

All partial derivatives are evaluated at the equilibrium state and constant entropy. Equation 1-12 can be written as [54]:

$$
p_{1}=A\left(\frac{\rho_{1}}{\rho_{0}}\right)+\frac{B}{2 !}\left(\frac{\rho_{1}}{\rho_{0}}\right)^{2}+\frac{C}{3 !}\left(\frac{\rho_{1}}{\rho_{0}}\right)^{3}+\cdots,
$$

in which

$$
\begin{gathered}
A=\rho_{0}\left(\frac{\partial p}{\partial \rho}\right)_{s, 0} \equiv \rho_{0} c_{0}^{2}, \\
B=\rho_{0}^{2}\left(\frac{\partial^{2} p}{\partial \rho^{2}}\right)_{s, 0} \\
C=\rho_{0}^{3}\left(\frac{\partial^{3} p}{\partial \rho^{3}}\right)_{s, 0}
\end{gathered}
$$

The parameter of nonlinearity, $\mathrm{B} / \mathrm{A}$ is defined as the ratio of the coefficients of the quadratic and the linear terms and equals:

$$
\frac{B}{A}=\frac{\rho_{0}}{c_{0}^{2}}\left(\frac{\partial^{2} p}{\partial \rho^{2}}\right)_{s, 0}
$$

The phase velocity $v_{\theta}$ can be written in terms of particle velocity, $v$ as:

$$
v_{\theta}=c_{0}+v+\frac{B}{2 A} v=c_{0}+\left(1+\frac{B}{2 A}\right) v .
$$

This equation indicates that the phase velocity is not constant and consists of two contributions: the wave velocity $\left(c_{0}\right)$ and the particle velocity $(v)[73,54]$. The coefficient of nonlinearity, $\beta$ is defined as: 


$$
\beta=1+\frac{B}{2 A}
$$

B/A has been mostly measured by finite-amplitude and thermodynamic methods [71]. The finite amplitude method is based on measuring the second harmonic generated in the medium [74]. The thermodynamic method is considered to be the most accurate way of measuring $\mathrm{B} / \mathrm{A}$. In this technique $\mathrm{B} / \mathrm{A}$ is expressed in terms of variation of speed of sound with respect to pressure and temperature by [71]:

$$
\frac{B}{A}=2 \rho_{0} c_{0}\left(\frac{\partial c}{\partial p}\right)_{T, 0}+\frac{2 \alpha_{V} c_{0} T}{c_{p}}\left(\frac{\partial c}{\partial T}\right)_{p, 0},
$$

where the first term is based on isothermal pressure changes and the second term is based on isobaric temperature changes [52]. Equation 1-20 shows that $\mathrm{B} / \mathrm{A}$ is a temperature dependent parameter.

Nonlinear effect manifests itself by distorting the wave and generating higher harmonics as the wave propagates through the medium. Nonlinearity is a cumulative effect which means that as the wave progresses, distortion increases. For a plane wave and in the absence of absorption, the degree of distortion is linearly proportional to frequency, source pressure, coefficient of nonlinearity $(\beta)$ and distance $[71,54]$. However, the frequency dependent attenuation damps higher frequency components of the wave more than lower ones.

A number of studies have shown the dependence of the nonlinear parameter on temperature for biological tissues in ex vivo measurements $[53,50,51]$, and it has been reported that $\mathrm{B} / \mathrm{A}$ can be used as a suitable parameter as a basis for temperature estimation $[53,52]$. Liu et al. presented the temperature dependence of B/A for porcine fat and porcine liver (Figure 1-6) [53]. They have demonstrated that the nonlinearity parameter could be used as a parameter to non-invasively estimate the temperature change in tissue. 

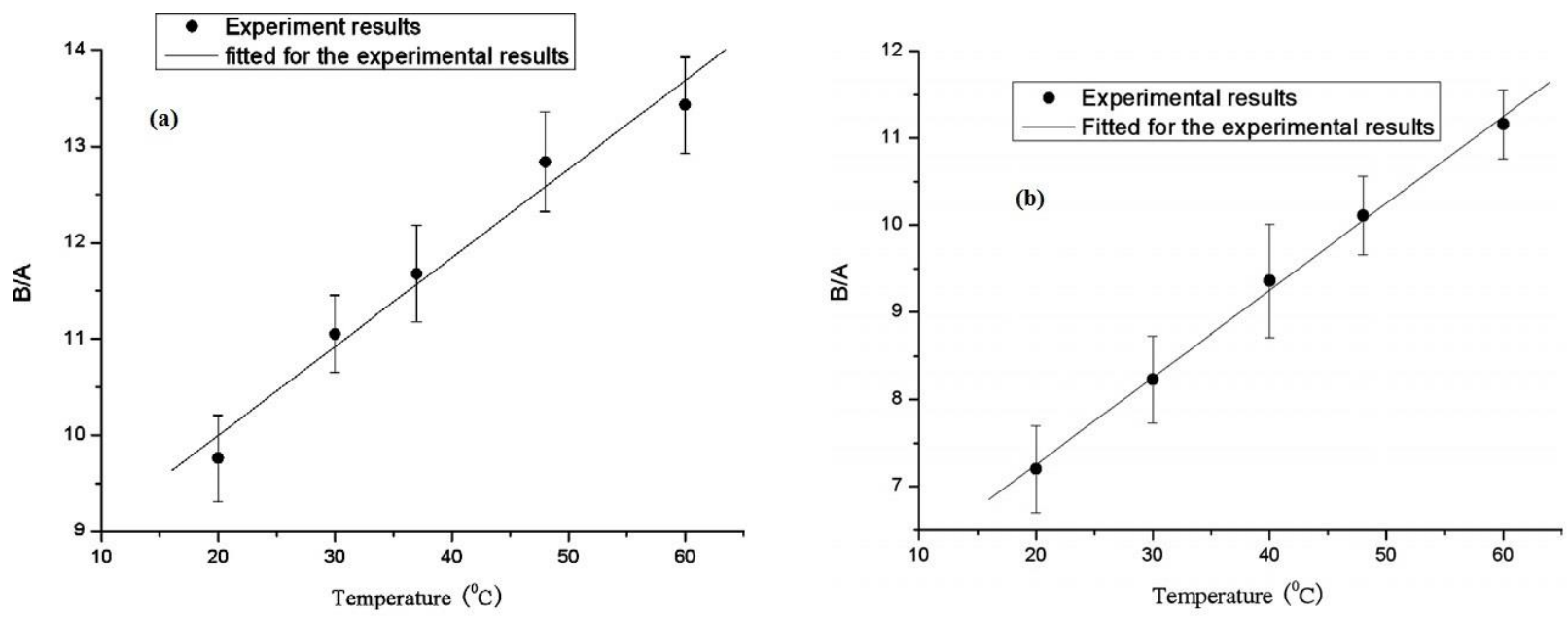

Figure 1-6: Temperature dependence of B/A of porcine (a) fat and (b) liver [53].

In a simulation study, van Dongen et al. showed that variation in temperature, changes the amplitudes of the harmonics of a transmitted acoustic wave in glycerol (Figure 1-7) [52]. They proposed a noninvasive thermometry method based on the amplitude ratios of harmonics generated by nonlinear wave propagation. In this technique a set of reference ratios $R_{i, j}$ are obtained which each is defined as the ratio between the amplitudes of the $\mathrm{i}^{\text {th }}$ and the $\mathrm{j}^{\text {th }}$ harmonic component. The reference ratios $\mathrm{R}_{\mathrm{i}, \mathrm{j}}$ are defined as:

$$
R_{i, j}(T, x)=\left\{\begin{array}{l}
\frac{F_{0}(T, x)}{H_{j}(T, x)} \text { for } i=1 \\
\frac{H_{i}(T, x)}{H_{j}(T, x)} \text { for } i>1
\end{array}\right.
$$

where $F_{0}, H_{i}$ and $H_{j}$ are the amplitudes of the fundamental frequency, $\mathrm{i}^{\text {th }}$ and the $\mathrm{j}^{\text {th }}$ harmonics at the propagation distance $x$. These reference ratios were obtained using simulations for a range of temperature in glycerol. Temperature at the propagation distance $x$ could be estimated by comparing the measured ratio at an unknown temperature with these reference ratios. 


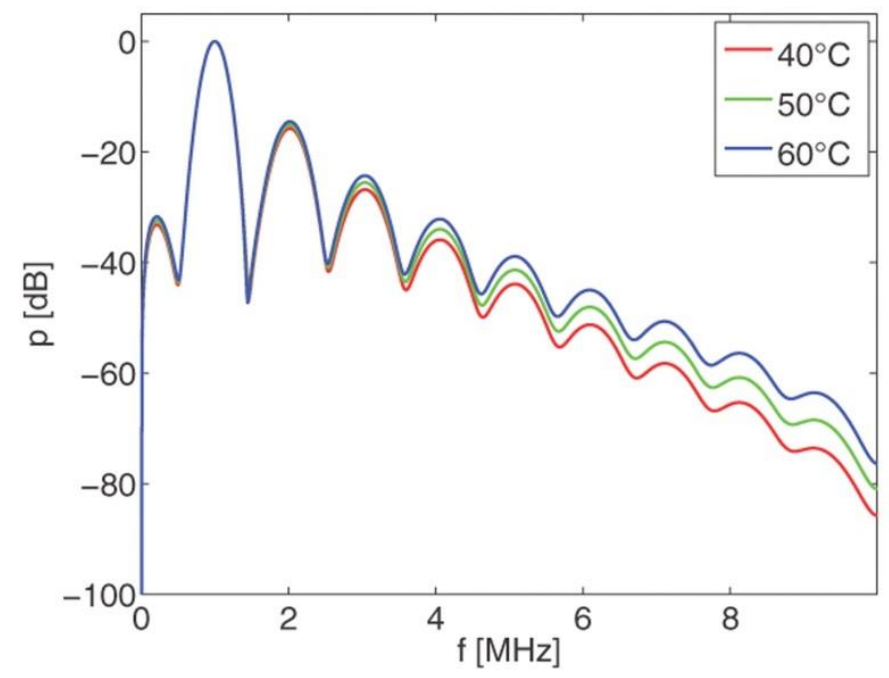

Figure 1-7: Simulated normalized frequency spectrum of an acoustic plane wave at distance $x=0.1 \mathrm{~m}$ in glycerol at temperatures $T=40^{\circ} \mathrm{C}, 50^{\circ} \mathrm{C}$ and $60^{\circ} \mathrm{C}$. The fundamental frequency is $1 \mathrm{MHz}$ [52].

There are several challenges in generating and detecting higher harmonics. In order to increase the degree of wave distortion both source pressure and frequency has to be increased. However, increasing the frequency results in a larger frequency-dependent attenuation which, in turn, dampens higher harmonics. Detecting multiple harmonics $\left(2^{\text {nd }}\right.$ and higher) requires a wide bandwidth for the ultrasound transducer and the electronic circuitry used in the signal's receiving path. For example, if a $13 \mathrm{MHz}$ transmit pulse is being used, the transducer and its associated electronics should be able to cover frequencies of up to at least $40 \mathrm{MHz}$ to be able to reliably detect the $2^{\text {nd }}$ and $3^{\text {rd }}$ harmonics of the transmit signal.

The method based on nonlinear propagation of the ultrasound beam, as a noninvasive thermometry technique, is less susceptible to tissue motion compared to the echo-shift technique, since it is a frequency-based method. Moreover, the technique has been reported to be more sensitive to temperature [52]. Therefore, an analysis on the temperature dependence of the amplitude of the harmonics and their ratios could provide a potential method for noninvasive temperature monitoring with a high sensitivity and low susceptibility to motion artifact. 


\subsection{Nonlinear Ultrasound Wave Models}

A mathematical model is needed to simulate the propagation of ultrasound wave in a medium in order to predict the acoustic filed generated by a transducer. The model needs to be capable of taking into account the nonlinear propagation of the sound, finite dimensions of the transducer and the dissipating effects of the medium [74].

The Khokhlov-Zabolotskaya-Kuznetsov (KZK) nonlinear wave equation is one of the most widely used mathematical models which describes the finite-amplitude propagation of the acoustic beam [74, 71]. The KZK equation is a parabolic approximation of the Westervelt nonlinear wave equation that consists of terms to account for diffraction, absorption and nonlinearity. The main assumptions in this model are that the sound source is directional and the wave varies slowly in the propagation direction. Therefore, this equation is accurate when the source dimensions is much bigger the wavelength ( $k a \gg 1$ where $k$ is the wave number and $a$ is the radius of the source), at field points not too close to the source surface and not far off the propagation axis (up to about $20^{\circ}$ off the axis) [75]. The KZK nonlinear wave equation can be given by:

$$
\frac{\partial^{2} p}{\partial z \partial \tau}=\frac{c_{0}}{2} \nabla_{\perp}^{2} p+\frac{\delta}{2 c_{0}^{3}} \frac{\partial^{3} p}{\partial \tau^{3}}+\frac{\beta}{2 \rho_{0} c_{0}^{3}} \frac{\partial^{2} p^{2}}{\partial \tau^{2}}
$$

where $\nabla_{\perp}^{2}=\partial^{2} / \partial x^{2}+\partial^{2} / \partial y^{2}$ is the transverse Laplacian that operates in a plane perpendicular to the beam axis. $\tau$ is the retarded time defined as $\tau=t-z / c_{0}$ and $\delta$ is the diffusivity of sound. The terms on the right-hand side of Eq. 1- 22 account for parabolic diffraction, thermoviscous absorption, and nonlinearity respectively.

The KZK equation reduces to the Burgers equation in the absence of diffraction $\left(\nabla_{\perp}^{2} p=\right.$ $0)$. The Burgers equation is the simplest model that describes the combined effects of 
nonlinearity and absorption for acoustic plane waves of finite amplitude [71]. The Burgers equation can be represented along $z$ axis as:

$$
\frac{\partial p}{\partial z}-\frac{\delta}{2 c_{0}^{3}} \frac{\partial^{2} p}{\partial \tau^{2}}=-\frac{\beta p}{\rho_{0} c_{0}^{3}} \frac{\partial p}{\partial \tau}
$$

where the lossless form can be evaluated by substituting $\delta=0$.

The Westervelt equation is a second-order nonlinear wave equation derived from the equation of motion and deformation equation which is given by:

$$
\nabla^{2} p-\frac{1}{c_{0}^{2}} \frac{\partial^{2} p}{\partial t^{2}}+\frac{\delta}{c_{0}^{4}} \frac{\partial^{3} p}{\partial t^{3}}=-\frac{\beta}{\rho_{0} c_{0}^{4}} \frac{\partial^{2} p^{2}}{\partial t^{2}} .
$$

The Westervelt equation provides a more complete description of nonlinear wave propagation than the KZK and Burgers models [71].

Several numerical methods have been developed to solve nonlinear wave equations which are divided into two categories of KZK-based models and other models [54, 71]. There are three general methods for solving the KZK equation: frequency domain, time domain, and combined time and frequency domain methods. An excellent review on different computational methods is given by Ginsberg and Hamilton (chapter 11) [71].

A phenomenological approach was introduced by Christopher and Parker that is not based on the KZK equation [76]. In this approach the effects of diffraction, nonlinearity and attenuation are solved independently over an incremental distance $\Delta z$ and the total pressure change can be approximated by their sum. This technique is called the operator splitting technique or the method of fractional steps. Tavakkoli et al. developed a second-order operator splitting technique in time domain using the Rayleigh integral $[77,78]$. 


\subsection{Thesis Hypothesis and Specific Aims}

The main objective of this research project is to develop a robust noninvasive and realtime temperature monitoring technique to measure the tissue temperature during hyperthermia. The hypothesis of this research is that the acoustic harmonics generated by nonlinear ultrasound wave propagation in tissue and/or tissue-like media are temperature dependent and they could be used to develop a more robust noninvasive thermometry technique in the presence of motion artifact compared to the conventional echo-shift thermometry method.

The specific aims of the proposed research are to:

1. Expand an existing KZK nonlinear ultrasound wave propagation model to make it temperature dependent.

2. Obtain the temperature dependence of the acoustic harmonics in water and in a tissuemimicking liquid using hydrophone measurements and compare the results with the expanded KZK simulation.

3. Obtain the temperature dependence of the acoustic harmonics in tissue-mimicking gel phantom and ex vivo muscle tissue in pulse echo mode with a clinical high-frequency ultrasound scanner.

4. Obtain 2D temperature maps using the nonlinear harmonics method in gel phantoms.

5. Study the motion artifact susceptibility of the harmonics and the echo-shift techniques.

\subsection{Overview of the Dissertation}

In Chapter 2, I investigated the temperature dependence of acoustic harmonics generated in water at various transmit pulse frequencies. A KZK nonlinear acoustic wave propagation model was expanded to include the temperature dependence of the medium parameters. 
Measurements were performed using two single-element transducers at two different transmit frequencies within the range of frequencies simulated. The simulation results were compared with measurements.

In Chapter 3, I presented the effect of temperature change on the generation of acoustic harmonics in a tissue mimicking liquid made of a mixture of $90 \%$ glycerol and $10 \%$ water (by volume). The temperature dependent KZK model was used to simulate nonlinear acoustic wave propagation in the medium in the temperature range of 26 to $46^{\circ} \mathrm{C}$. Two single-element focused transducers with center frequencies of 5 and $13 \mathrm{MHz}$ were used to transmit finite amplitude ultrasound signals. The axial harmonic profiles were obtained and the change in their peak values with temperature was analyzed for both frequencies.

In chapter 4, I examined the change with temperature in pressure amplitude and the energy content of the backscattered fundamental frequency, the second and the third harmonics in tissue-mimicking gel phantoms and ex vivo bovine muscle tissues. A commercial highfrequency ultrasound imaging system was used to generate and detect acoustic harmonics in pulse-echo mode.

In chapter 5, I explored the feasibility of obtaining $2 \mathrm{D}$ temperature maps in a tissuemimicking gel phantom by estimating the change in backscattered energy of the acoustic harmonics and the conventional RF echo-shift technique. The sensitivity of both techniques to a motion artifact was studied by using a pulsatile pump to induce motion.

Chapter 6 summarizes the results and future work. Appendix A summarizes all the contributions resulting from the research work to date, that both directly and indirectly contributed to this dissertation. 


\section{Chapter 2: Temperature dependence of acoustic harmonics generated by nonlinear ultrasound wave propagation in water at various frequencies ${ }^{1}$}

\subsection{Abstract}

Ultrasound-based thermometry requires a temperature-sensitive acoustic parameter that can be used to estimate the temperature by tracking changes in that parameter during heating. The objective of this study is to investigate the temperature dependence of acoustic harmonics generated by nonlinear ultrasound wave propagation in water at various pulse transmit frequencies from 1 to $20 \mathrm{MHz}$. Simulations were conducted using an expanded form of Khokhlov-Zabolotskaya-Kuznetsov (KZK) nonlinear acoustic wave propagation model in which temperature dependence of the medium parameters was included. Measurements were performed using single-element transducers at two different transmit frequencies of 3.3 and 13 $\mathrm{MHz}$ which are within the range of frequencies simulated. The acoustic pressure signals were measured by a calibrated needle hydrophone along the axes of the transducers. The water temperature was uniformly increased from $26^{\circ} \mathrm{C}$ to $46^{\circ} \mathrm{C}$ in increments of $5^{\circ} \mathrm{C}$. The results show that the temperature dependence of the harmonic generation is different at various frequencies which is due to the interplay between the mechanisms of absorption, nonlinearity and focusing gain. At the transmit frequencies of 1 and $3.3 \mathrm{MHz}$, the harmonic amplitudes decrease with

\footnotetext{
${ }^{1}$ This chapter is based on an original article published as: B. Maraghechi, M. H. Hasani, M. C. Kolios, J. Tavakkoli, "Temperature dependence of acoustic harmonics generated by nonlinear ultrasound wave propagation in water at various frequencies," The Journal of the Acoustical Society of America, vol. 139, no. 5, pp. 2474-2481, May 2016.
} 
increasing the temperature, while the opposite temperature dependence is observed at 13 and 20 MHz.

\subsection{Introduction}

Noninvasive thermometry is needed to monitor the temperature change in the heated region during thermal treatments in order to improve the treatment efficacy and safety. Ultrasound is an attractive option for temperature mapping since it is non-ionizing, portable and is inexpensive. Several ultrasonic techniques have been proposed for noninvasive tissue thermometry $[41,38]$. In order to noninvasively estimate the temperature using ultrasound, a temperature sensitive acoustic parameter is required. The temperature could be estimated by knowing the temperature dependence of the parameter and tracking changes in that parameter during heating.

Acoustic pulses with relatively high-pressure progressively get distorted as they propagate and generate higher harmonics. This effect increases with increasing frequency, source pressure and focusing gain of the beam at a given propagation distance [71]. Finite-amplitude effects of nonlinear propagation of ultrasound waves are used in various diagnostic and therapeutic applications of ultrasound [73, 79].

Tissue Harmonic Imaging (THI) is an imaging technique in which a pulse with a center frequency of $f_{0}$ is transmitted and the signal containing the second harmonic $\left(2 \times f_{0}\right)$ is received to form an image $[73,79]$. Superharmonic imaging uses the combination of the third, fourth and the fifth harmonics of the received signal to image [80]. It has been shown that imaging with these harmonics improves the spatial resolution and reduces the aberration and reverberation artifacts of the image $[73,79,80]$. 
Acoustic nonlinearity parameter imaging has been applied for tissue characterization. The value of the acoustic nonlinearity parameter (B/A) of fatty tissues is typically twice that of nonfatty tissues [81]. Zhang et al. showed that B/A can provide information about the composition of the tissue [81]. They generated B/A images of biological specimens such as porcine normal and fatty liver tissues, and porcine fat tissue.

Studies have shown that B/A is temperature dependent and it could be considered as a basis for an ultrasound-based method for noninvasive temperature estimation [52, 53]. The B/A is about four times more temperature sensitive compared to the speed of sound and thermal expansion in water [52]. van Dongen and Verweij simulated the nonlinear propagation of acoustic waves in glycerol using the lossless form of the Burgers equation [52]. They showed that the harmonic pressure amplitudes are temperature dependent and could potentially be considered as suitable parameters for noninvasive ultrasound thermometry. However, the lossless form of Burgers equation is the simplest model that describes only the effect of nonlinearity for acoustic plane waves of finite amplitude. Both simulated an acoustic plane wave propagating in water in order to study the temperature dependence of harmonics [82]. The simulations were performed using two different solutions of Burgers equation known as Keck-Beyer and Fubini for lossy and lossless media, respectively [71, 82]. A monochromatic $1 \mathrm{MHz}$ source with a pressure of $1 \mathrm{MPa}$ was used in the study. The author showed that the harmonics are weakly sensitive to temperature and a minimum in the harmonic pressure value occurs at around $45^{\circ} \mathrm{C}$. It is worth noting that neither of the simulation studies mentioned did take into account the combined effects of diffraction, absorption and nonlinearity - all of which change the harmonic amplitude of the propagating waves. 
We have recently shown that the pressure amplitude and the energy content of the backscattered fundamental frequency, the second and the third harmonics generated by nonlinear ultrasound propagation in tissue-mimicking gel phantoms and ex vivo bovine muscle tissues are highly sensitive to temperature, when the acoustic harmonics were generated by transmitting a $13 \mathrm{MHz}$ pulse using a high-frequency ultrasound imaging scanner [83]. It was also demonstrated that higher harmonics have higher sensitivity to temperature.

The Khokhlov-Zabolotskaya-Kuznetsov (KZK) nonlinear wave equation is a wellestablished model for finite-amplitude wave propagation $[71,84,74,54]$. The KZK equation is a parabolic approximation of the Westervelt nonlinear wave equation that consists of terms to account for diffraction, absorption and nonlinearity [71]. The diffraction term accounts for the finite dimensions of the source and the attenuation term considers the heat conduction and viscosity of the medium. The nonlinear term accounts for the nonlinear propagation of the finiteamplitude wave.

In this study, the temperature dependence of harmonic amplitudes generated by nonlinear ultrasound wave propagation in water is examined at various pulse transmit frequencies from 1 to $20 \mathrm{MHz}$ using the KZK wave equation. Nonlinear ultrasound wave propagation simulations were performed using a time-domain numerical solution of a modified KZK nonlinear wave equation that accounts for temperature dependent medium parameters. The simulation results are compared with measurements. Measurements were performed using two single element transducers at frequencies of $3.3 \mathrm{MHz}$ and $13 \mathrm{MHz}$.

The main objective of this study is to investigate and understand the mechanisms of action of harmonics generation as a function of temperature at various frequencies in water which is a simple, homogeneous and more controlled medium compared to tissue. Soft tissues 
exhibit additional complexities such as significant structural heterogeneity that change as a function of time (tissue decay) and temperature. Moreover, water was chosen in experiments and simulations because the values for acoustic parameters (attenuation coefficient, speed of sound, density and $\mathrm{B} / \mathrm{A}$ ) of water were available as a function of temperature in the literature. Currently, there are limited amount of information on the temperature dependence of these acoustic parameters for soft tissue which we need as inputs to our simulation model $[53,50,51]$.

\subsection{Materials and Method}

\subsubsection{Temperature Dependent Nonlinear Ultrasound Wave Propagation Model}

Nonlinear ultrasound wave propagation simulations were performed using a time-domain numerical solution of a modified KZK nonlinear wave equation in which temperature dependence of the medium parameters was included. The dimensionless form of the temperature-dependent KZK equation for focused sources could be given as [74]:

$$
\frac{\partial P}{\partial \sigma}=\frac{1}{4 G(T)} \int_{-\infty}^{\tau}\left(\frac{b}{a} \frac{\partial^{2} P}{\partial X^{2}}+\frac{a}{b} \frac{\partial^{2} P}{\partial Y^{2}}\right) d \tau^{\prime}+A(T) \frac{\partial^{2} P}{\partial \tau^{2}}+N(T)\left(P \frac{\partial P}{\partial \tau}\right),
$$

where $P$ is the transformed source pressure amplitude defined as $P=\left(p / p_{0}\right)$ which $p$ is the sound pressure in the Cartesian coordinates and $p_{0}$ is the source pressure amplitude. $\sigma=z / d$ is the dimensionless $z$ axis (axial distance divided by the focal length $d$ ) for a source with characteristic sizes of $a$ and $b$ in the $x$ and $y$ directions with an angular frequency of $\omega_{0} . X$ and $Y$ are the transformed transverse coordinates $(X=x / a, Y=y / b)$ and $T$ is the temperature. $G(T)$ is the focusing gain defined as:

$$
G(T)=\frac{z_{0}(T)}{d}=\frac{\pi f_{0} a b}{c_{0}(T) d}
$$


where $z_{0}$ is the Rayleigh distance. $A(T)$ is the absorption parameter defined as:

$$
A(T)=\alpha(T) d=\alpha_{0}(T) f_{0}^{2} d
$$

where $\alpha_{0}$ is the pressure absorption coefficient. $N(T)$ is the nonlinear parameter defined as:

$$
N(T)=\frac{d}{\bar{z}(T)}=\frac{\beta(T) 2 \pi f_{0} p_{0}}{\rho_{0}(T) c_{0}^{3}(T)} d,
$$

where $\bar{z}$ is the plane wave shock formation distance. $c_{0}$ and $\rho_{0}$ are the small signal speed of sound and the medium density, respectively. $\tau$ is the transformed retarded time defined as $\tau=\omega_{0} t^{\prime}$ where $t^{\prime}$ is the retarded time $\left(t^{\prime}=t-z / c_{0}\right)$. The terms on the right-hand side of Eq. $2-1$ account for diffraction, nonlinearity, and thermoviscous absorption, respectively [74].

The source boundary condition was defined as $p=p_{0} f\left(t+x^{2} / 2 c_{0} d_{x}+y^{2} / 2 c_{0} d_{y}\right) g(x, y)$ $\xi(x, y), z=0$, where $f(t), g(x, y)$ and $\xi(x, y)$ are the source temporal excitation, the source spatial aperture function, and the source apodization function, respectively. By using the variable transformations and dimensionless variables, the source boundary condition could be rewritten as $P=f\left(\tau+G_{x} X^{2}+G_{y} Y^{2}\right) g(X, Y) \xi(X, Y), \sigma=0$, where $G_{x}=G b d / a d_{x}$ and $G_{y}=G a d / b d_{y}$ are the directional focusing gains in $x$ and $y$ directions, respectively.

For a circular source, the function $g(X, Y)$ can be written as:

$$
g(X, Y)=\left\{\begin{array}{cc}
1 & X^{2}+Y^{2} \leq 1 \\
0 & \text { otherwise }
\end{array}\right.
$$

In the selection of boundary values, the extent of spatial domains, $X_{\max }$ and $Y_{\max }$, were chosen large enough to minimize reflections due to artificial boundary conditions. The time window was also large enough to encompass the entire waveform, including delayed edge waves [74].

Nonlinear propagation of 9 and 7 cycle pulses at $3.3 \mathrm{MHz}$ and $13 \mathrm{MHz}$ in water with source pressure amplitudes of $0.26 \mathrm{MPa}$ and $0.05 \mathrm{MPa}$, respectively, and for the same transducer 
geometries used in our experiments were simulated. The source pressure amplitudes used in simulations were selected in order to achieve similar pressure and similar degree of nonlinear waveform distortion as to that obtained in the experiments at the transducer focus and along the acoustic axes when water was at the baseline temperature of $26^{\circ} \mathrm{C}$. Simulations for the $1 \mathrm{MHz}$ and the $20 \mathrm{MHz}$ pulses were performed using the same parameters as those at 3.3 and $13 \mathrm{MHz}$, respectively. The source pressure waveform was a Gaussian envelope pulse given as:

$$
P=\exp \left[-\left(\frac{\tau}{n \pi}\right)^{2}\right] \sin \tau
$$

where $n$ is the number of cycles. In order to replicate the effects induced by raising the temperature of water from $26^{\circ} \mathrm{C}$ to $46^{\circ} \mathrm{C}$, the empirical temperature dependent values of four acoustic medium parameters at each temperature were used as an input to the simulation. The values of sound speed, medium's density, absorption coefficient and the B/A as a function of temperature in water were obtained from published data and are shown in Figure 2-1 [52, 54, 85].

In order to take into account the spatial averaging effect of the hydrophone, harmonics were averaged over a circular area corresponding to virtual area of the hydrophone used in the experiments $[86,87]$. The change in the peak pressure amplitudes of the harmonics and their ratios were analyzed at each simulated water temperature from $26^{\circ} \mathrm{C}$ to $46^{\circ} \mathrm{C}$ in increments of $5^{\circ} \mathrm{C}$. 

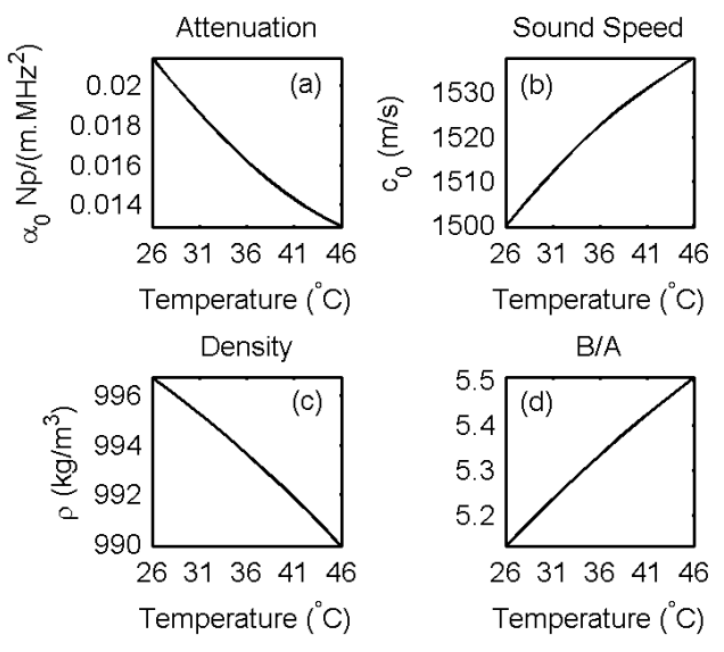

Figure 2-1: The (a) attenuation coefficient [54], (b) speed of sound [54], (c) density [52], and (d) B/A [85] as empirical functions of temperature in water.

\subsubsection{Experiments}

A broadband single-element transducer with center frequency of $5 \mathrm{MHz}$ (Matec, Northborough, MA, USA) was used to generate pulses at $3.3 \mathrm{MHz}$. The transducer had a diameter of $6 \mathrm{~mm}$ and a focal length of $3 \mathrm{~cm}$. The transducer was driven by an arbitrary function generator (Model AFG3101; Tektronix, Beaverton, OR, USA) through a class-A broadband RF power amplifier (Model 325LA; E\&I, Rochester, NY, USA), with frequency range of $250 \mathrm{kHz}-$ $150 \mathrm{MHz}$ and nominal gain of $50 \mathrm{~dB}$. A high-frequency ultrasound imaging scanner (Vevo® 770, Visualsonics Inc., Toronto, ON, Canada) with a $25 \mathrm{MHz}$ center frequency wide-band single-element transducer (RMV-710B, $7.1 \mathrm{~mm}$ diameter, $15 \mathrm{~mm}$ focal length) was used to generate pulses at $13 \mathrm{MHz}$. Acoustic harmonics were generated by transmitting pulse trains of 15-cycle length at 3.3 and $13 \mathrm{MHz}$ with focal positive peak pressures of approximately $0.6 \mathrm{MPa}$ and $0.9 \mathrm{MPa}$, respectively in water at $26^{\circ} \mathrm{C}$. The purpose of choosing these pressures was to have a reasonable SNR values for the first, second and third harmonics. 
The acoustic pressure signals were measured along the axes of the transducers by a calibrated needle hydrophone with an active element diameter of $400 \mu \mathrm{m}$ (Model HNA-0400; ONDA Corporation, Sunnyvale, CA, USA). Water was placed in a $2.5 \mathrm{~cm}$ diameter cylindrical container with a transparent window at the bottom. Water temperature was uniformly elevated from $26^{\circ} \mathrm{C}$ to $46^{\circ} \mathrm{C}$ in increments of $5^{\circ} \mathrm{C}$ by the flow of hot water around the container. Hot water was pumped from a controlled circulating water bath (Haake DC10, Thermo Electron Corp., Newington, NH) using a peristaltic pump (Masterflex ${ }^{\circledR}$ L/S®, Cole Parmer, Chicago, IL). The 5 MHz transducer was immersed in a water tank for coupling with the container. The RMV-710B transducer was coupled with the container using acoustic gel. A schematic of the experimental setup is shown in Figure 2-2. The received signal detected by the hydrophone was recorded with a $350 \mathrm{MHz}$ digital oscilloscope (Model 7032A; Agilent Technologies, Santa Clara, CA, USA) and analyzed offline. The pressure amplitudes of the fundamental frequency $\left(p_{I}\right)$, and its harmonics (second $\left(p_{2}\right)$, third $\left.\left(p_{3}\right)\right)$ generated by nonlinear ultrasound propagation were obtained by calculating the frequency spectrum of the measured acoustic pressure signals. The peak pressure harmonic amplitudes and their ratios $\left(p_{2} / p_{1}\right.$ and $\left.p_{3} / p_{1}\right)$ were analyzed as a function of water temperature and transducers transmit frequencies. The experiments were repeated twelve times at each transmit frequency and temperature. 


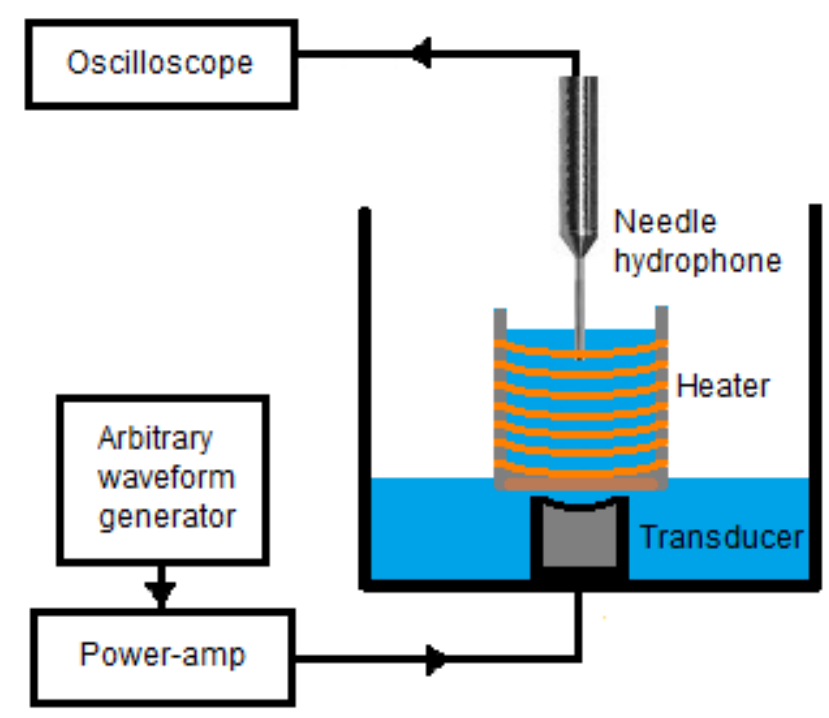

Figure 2-2: Schematic of the experimental setup at 3.3 MHz.

\subsection{Results}

The simulated and the measured waveforms and their frequency spectra at both transmit frequencies of 3.3 MHz and $13 \mathrm{MHz}$ are shown in Figure 2-3 and Figure 2-4, respectively. The measured and simulated pressure amplitudes of the lowest three harmonic components along the axis of the ultrasound beam with transmit frequencies of $3.3 \mathrm{MHz}$ and $13 \mathrm{MHz}$ are shown in Figure 2-5. The average and standard deviation of the harmonic amplitudes and their ratios were calculated for each temperature. Figure 2-6 and Figure 2-7 show the simulated and measured percentage changes in the pressure harmonic amplitudes $\left(p_{1}, p_{2}\right.$ and $\left.p_{3}\right)$ and their ratios $\left(p_{2} / p_{1}\right.$ and $\left.p_{3} / p_{1}\right)$, respectively at various frequencies. 

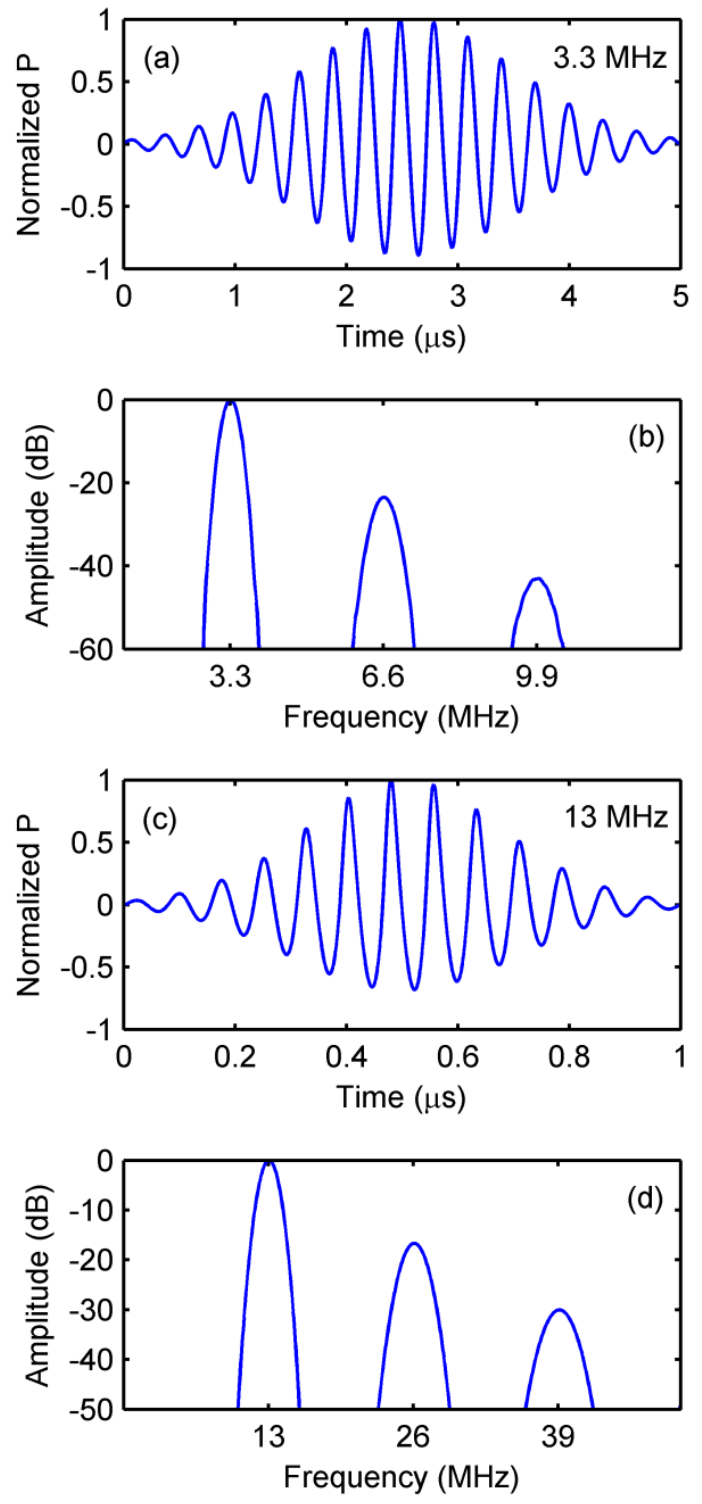

Figure 2-3: Simulated normalized focal pressures and the magnitude of their spectra for pulses with transmit frequencies of (a) and (b) $3.3 \mathrm{MHz}$ and (c) and (d) at $13 \mathrm{MHz}$, respectively at the initial temperature $\left(26^{\circ} \mathrm{C}\right)$. 

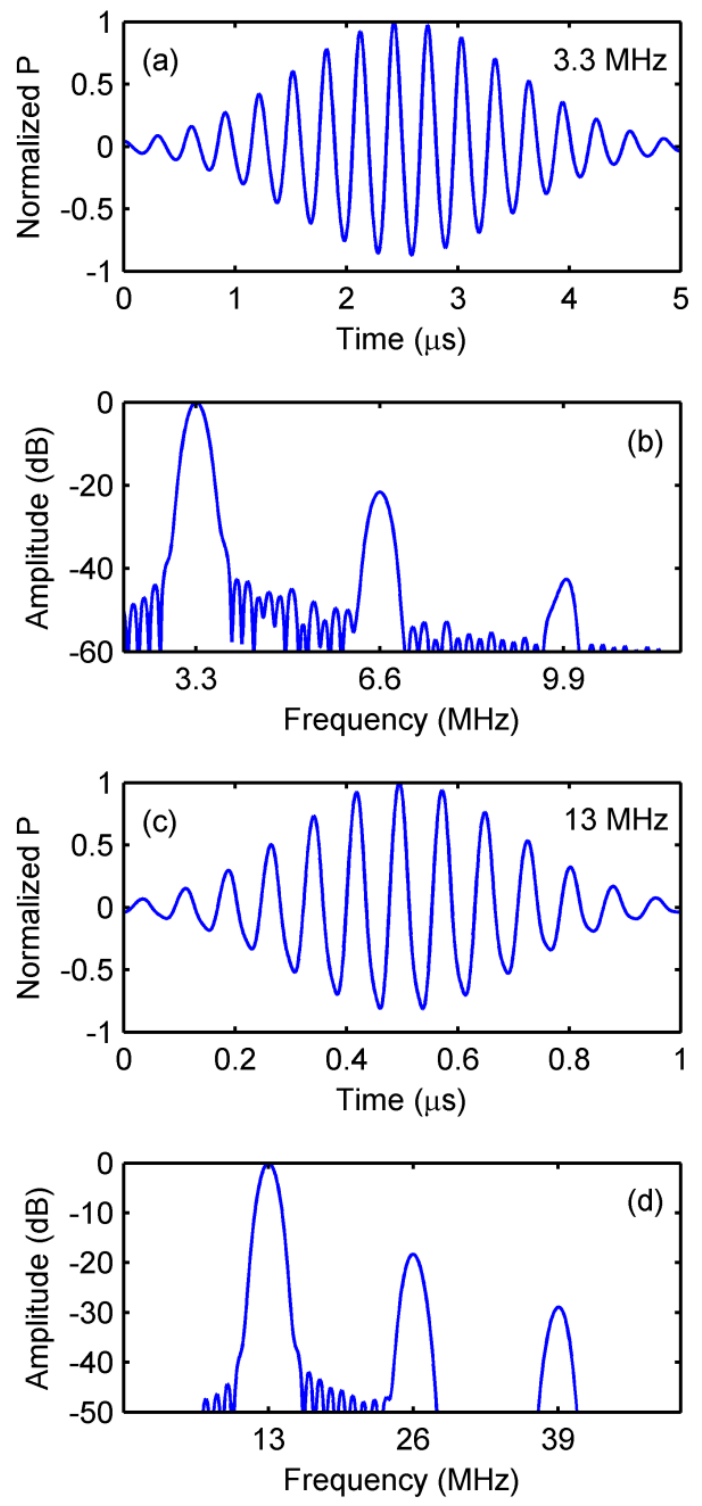

Figure 2-4: Measured normalized focal pressures and the magnitude of their spectra for pulses with transmit frequencies of (a) and (b) $3.3 \mathrm{MHz}$ and (c) and (d) $13 \mathrm{MHz}$, respectively at the initial temperature $\left(26^{\circ} \mathrm{C}\right)$. 

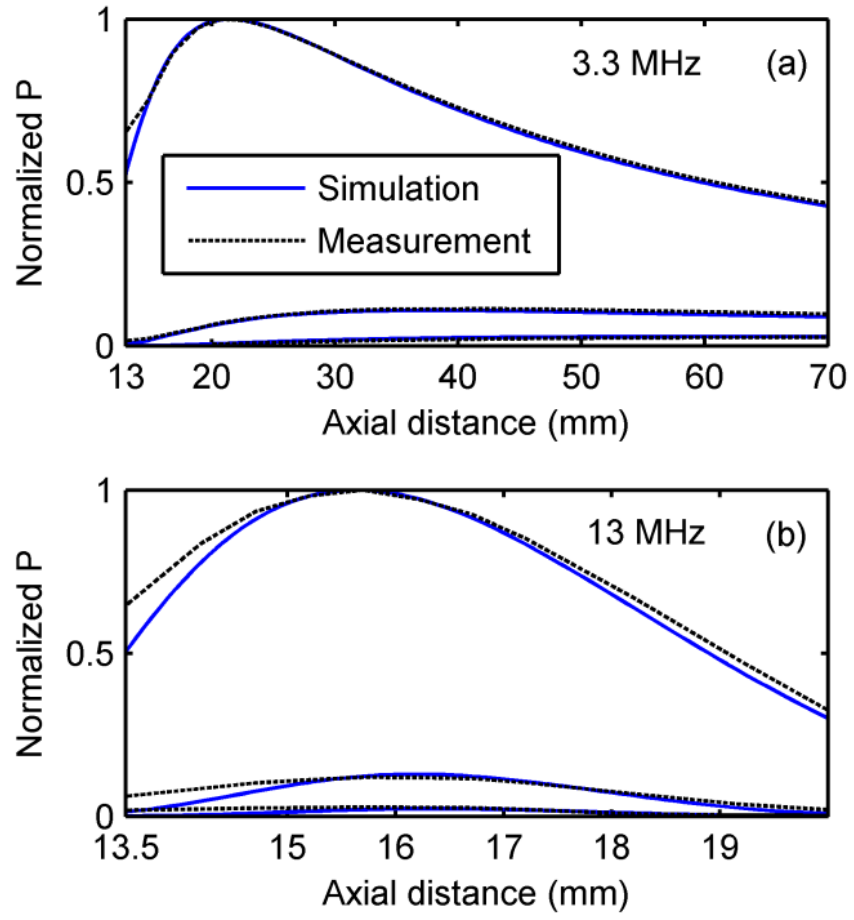

Figure 2-5: Measured (dotted) and simulated (solid) normalized pressure amplitudes of the lowest three harmonic components along the axis of the beam with transmit frequencies of (a) $3.3 \mathrm{MHz}$ and (b) $13 \mathrm{MHz}$ at the initial temperature $\left(26^{\circ} \mathrm{C}\right)$. 

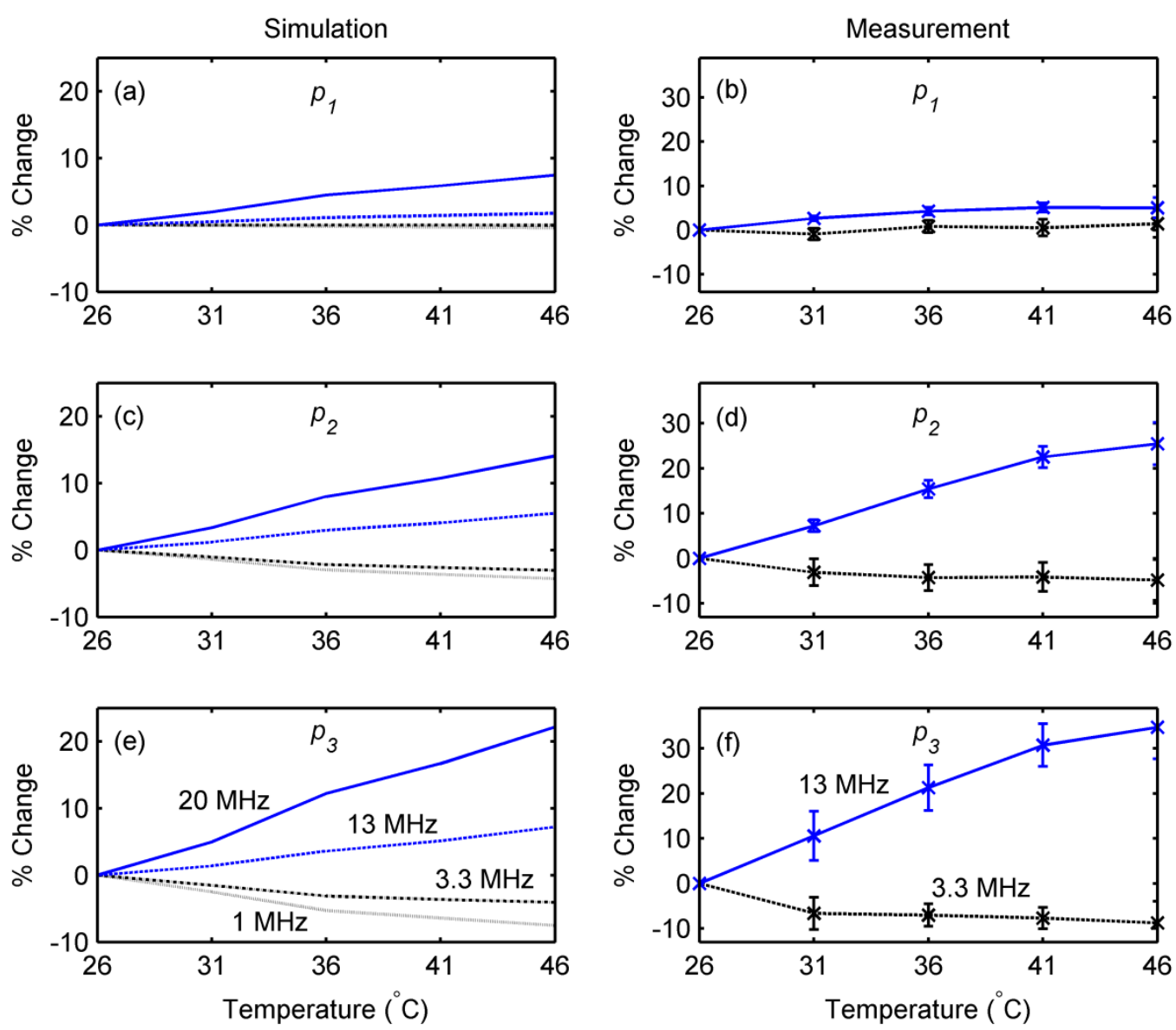

Figure 2-6: Simulated (left column) and measured (right column) changes in (a) and (b) $p_{1}$, (c) and (d) $p_{2}$, (e) and (f) $p_{3}$ as a function of temperature with respect to the initial temperature $\left(26^{\circ} \mathrm{C}\right)$ for pulses with transmit frequencies of 1, 3.3, 13 and $20 \mathrm{MHz}$. Measurements (right column) were only performed at two frequencies of $3.3 \mathrm{and} 13 \mathrm{MHz}$. The error bars represent the standard deviation of 12 independent trials. 

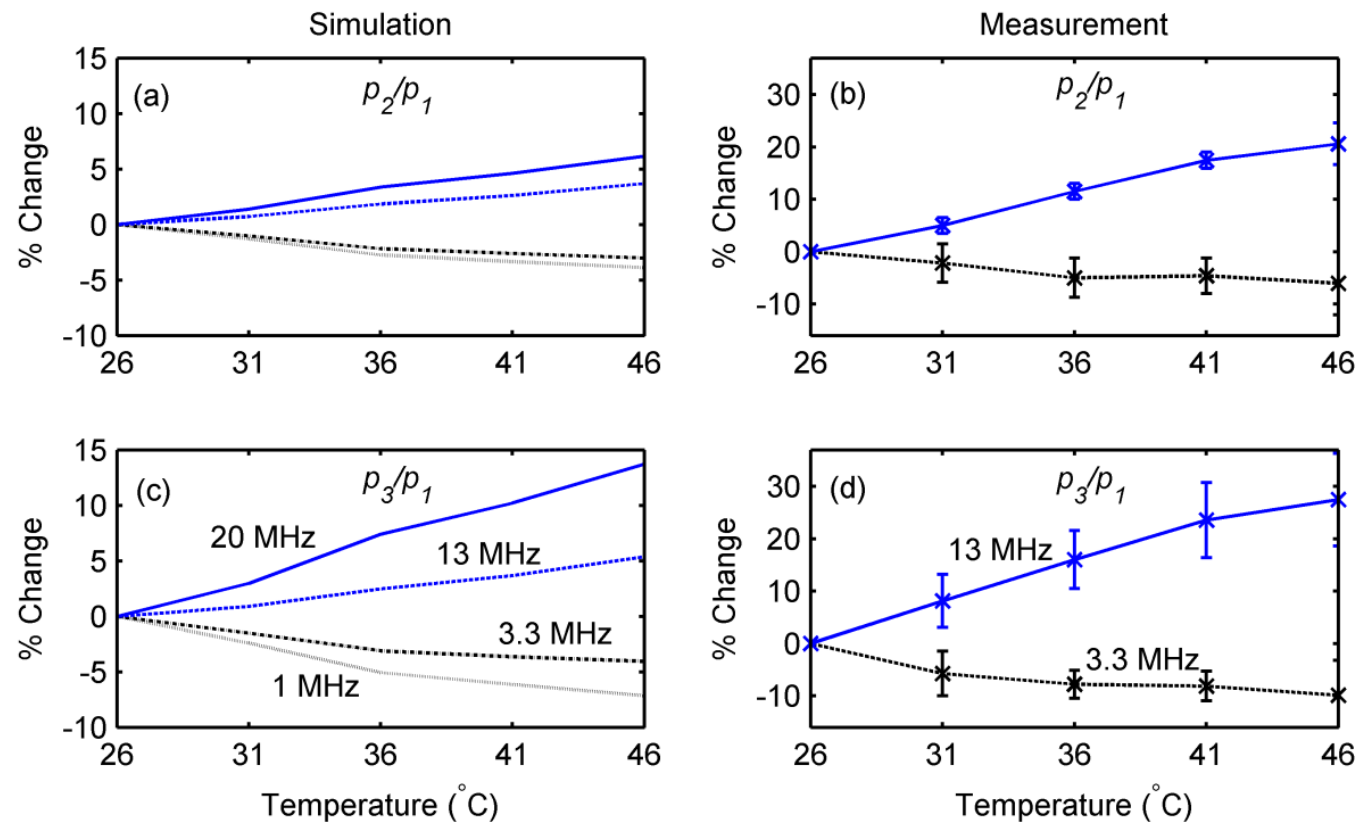

Figure 2-7: Simulated (left column) and measured (right column) changes in (a) and (b) $p_{2} / p_{1}$, (c) and (d) $p_{3} / p_{1}$ as a function of temperature with respect to the initial temperature $\left(26^{\circ} \mathrm{C}\right)$ for pulses with transmit frequencies of $1,3.3$, 13 and $20 \mathrm{MHz}$. Measurements (right column) were only performed at two frequencies of 3.3 and $13 \mathrm{MHz}$. The error bars represent the standard deviation of 12 independent trials.

\subsection{Discussion}

The results obtained from measurements and simulations shown in Figure 2-6 and Figure 2-7 demonstrate that the harmonic pressure amplitudes and their ratios decrease with temperature for the pulse transmit frequency of 1 and $3.3 \mathrm{MHz}$. On the other hand, at 13 and 20 $\mathrm{MHz}$ transmit frequencies, the harmonics and their ratios show an increasing trend as the temperature increases from $26^{\circ} \mathrm{C}$ to $46^{\circ} \mathrm{C}$.

The results can be explained by the interplay between the temperature dependence of the absorption, nonlinearity and focusing gain. The relative importance of nonlinearity and absorption in the KZK model used in this study is determined by the dimensionless parameters of nonlinearity $(N)$ and absorption $(A)$, respectively. The ratio $\Gamma=N / A$ is defined as the Gol'dberg number, which provides a measure of balance between the nonlinear and the 
absorption processes [84]. Duck stated that when $\Gamma \gg 1$ the nonlinear process dominates and when $\Gamma$ is close to 1.0 , the effects of nonlinearity and absorption are comparable [73]. The focusing gain $(G)$ is the peak value of $p / p_{0}$ at the geometric focus in the absence of absorption and nonlinearity [84]. Figure 2-8 shows the percentage changes in $A, N, \Gamma$ and $G$ as a function of temperature for pulse transmit frequencies of 1,3.3, 13 and $20 \mathrm{MHz}$.
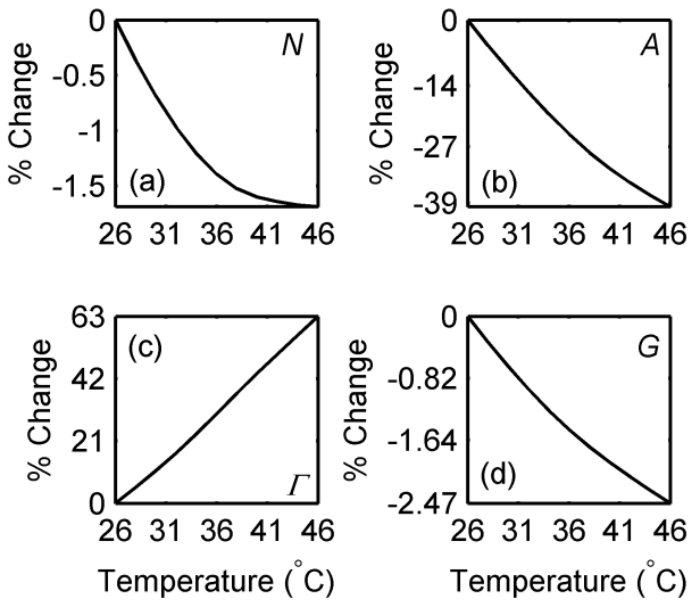

Figure 2-8: The percentage changes in (a) the nonlinear parameter $(N)$, (b) the absorption parameter (A), (c) the Gol'dberg number $(\Gamma)$ and (d) the focusing gain $(G)$ as a function of temperature for pulses with transmit frequencies of 1, 3.3, 13 and $20 \mathrm{MHz}$.

As the temperature increases from $26^{\circ} \mathrm{C}$ to $46^{\circ} \mathrm{C}$, the absorption parameter decreases by about $40 \%$ whereas the nonlinear parameter and the focusing gain decrease by about $-1.6 \%$ and $2.5 \%$, respectively (shown in Figure 2-8). A decrease in attenuation enhances the generation of harmonics but a decrease in nonlinearity reduces the amount of wave distortion due to nonlinear propagation (and therefore the creation of harmonics). A Decrease in the focusing gain reduces the pressure at the focus and causes reduction in harmonic generation. For the $1 \mathrm{MHz}$ and 3.3 $\mathrm{MHz}$ pulses the Gol'dberg number is about 81 and 24 , respectively at $26^{\circ} \mathrm{C}$. This indicates that the effects of nonlinearity significantly dominate over absorption. Therefore, at low frequencies 
the decrease in the harmonics with temperature is due to the combined effects of reduction in both the focusing gain and nonlinearity with temperature.

As the frequency increases, the effects of absorption become more pronounced and the Gol'dberg number decreases. The value of Gol'dberg number is equal to 1.2 and 0.7 at $26^{\circ} \mathrm{C}$ for pulses with transmit frequencies of 13 and $20 \mathrm{MHz}$, respectively. Although at high frequencies both the focusing gain and the nonlinearity decrease with temperature, the reduction of attenuation compensates their effects. Therefore, at high frequencies the harmonics increase with temperature due to the significant decrease in absorption as a function of temperature. The change of harmonics with temperature better follow the temperature dependence of the Gol'dberg number.

Figure 2-6 and Figure 2-7 show that higher harmonics have higher sensitivity to temperature change. For the 1 and $3.3 \mathrm{MHz}$ transmit frequencies, the higher the harmonic number, the steeper the decrease of the harmonics amplitude and their ratios with temperature. On the other hand, at 13 and $20 \mathrm{MHz}$, the higher the harmonic number, the steeper is the slope of the increase in the harmonic amplitudes and their ratios as the temperature increases from $26^{\circ} \mathrm{C}$ to $46^{\circ} \mathrm{C}$. Keck and Beyer have shown that for a finite-amplitude plane wave propagating in an attenuating medium, the $\mathrm{n}^{\text {th }}$ harmonic pressure amplitude is proportional to $\left[\beta / \alpha_{0} \rho_{0} c_{0}^{3}\right]^{n-1}[71$, 88]. Therefore, the temperature dependence of the acoustic parameters manifests themselves more prominently in the higher harmonics. Figure 2-9 shows the percentage change of $[\beta /$ $\left.\alpha_{0} \rho_{0} c_{0}^{3}\right]^{n-1}$ as a function of temperature for the harmonics at $13 \mathrm{MHz}$. This demonstrates that the higher harmonics are more sensitive to temperature. 


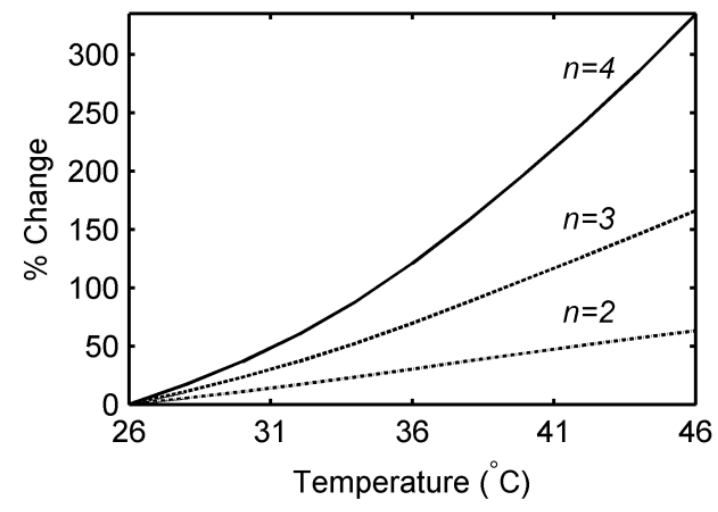

Figure 2-9: The percentage change of $\left[\boldsymbol{\beta} / \boldsymbol{\alpha}_{\mathbf{0}} \boldsymbol{\rho}_{\mathbf{0}} \boldsymbol{c}_{\mathbf{0}}^{3}\right]^{n-1}$ as a function of temperature for the second $(n=2)$, third $(n=3)$ and fourth $(n=4)$ harmonics at $13 \mathrm{MHz}$ transmit pulse frequency.

The increasing trend of the harmonics and their ratios with temperature at $13 \mathrm{MHz}$ are consistent with those obtained in tissue-mimicking gel phantoms and ex vivo bovine muscle at the same transmit frequency [83].

Figure 2-6 and Figure 2-7 demonstrate that the trend of harmonics amplitudes and their ratios with temperature is similar in both the measurements and simulations. The results obtained from simulations at $3.3 \mathrm{MHz}$ are within the standard deviation of the results obtained from measurements. However, the difference in the magnitude of the changes between simulations and experiments is more significant at $13 \mathrm{MHz}$. This could be due to approximations in values of acoustic parameters of water as a function of temperature as input in the simulations. The differences between measurements and simulations could also be due to measurement errors, since the acoustic field was manually scanned. The variation of frequency response with temperature of a typical hydrophone, such as the one we used in our study, could also be a possible reason for the mismatch between measurements and simulations. 


\subsection{Conclusion}

In this work, the temperature dependence of acoustic harmonics generated by nonlinear ultrasound wave propagation was investigated in water for a range of pulse transmit frequencies from 1 to $20 \mathrm{MHz}$. Measurements were conducted using two single element transducers to transmit the acoustic pressure signals and a calibrated hydrophone to receive them. The temperature-dependent KZK nonlinear ultrasound beam simulations were performed for the same experimental geometry. Several conclusions can be drawn from the results obtained in this study:

1. The harmonics generated by nonlinear ultrasound wave propagation in water are temperature dependent.

2. The temperature dependence of the harmonics generation is different for various transmit pulse frequencies. The temperature dependence of harmonics generated by nonlinear ultrasound wave propagation decreases with increasing temperature at 1 and $3.3 \mathrm{MHz}$ while the opposite temperature dependence is observed at 13 and $20 \mathrm{MHz}$. The harmonics and the harmonic ratios at 1 and $20 \mathrm{MHz}$ are more sensitive to temperature change compared to those at 3.3 and $13 \mathrm{MHz}$.

3. The temperature dependence of harmonics depends on absorption, nonlinearity and focusing gain.

4. For a given transmit frequency, the higher the harmonic number, the higher is its sensitivity to temperature.

5. The change in the harmonic generation as a function of temperature shows similar trends in both simulations and experiments at 3.3 and $13 \mathrm{MHz}$. However, there is a discrepancy between simulation and measurement results at $13 \mathrm{MHz}$. 
It should be noted that the conclusions mentioned above depends strongly on the source parameters (transducer geometry, source pressure amplitude and frequency) used in this study. In particular, characteristics of a nonlinear field depend on the source pressure amplitude. Varying the source pressure amplitude could, therefore, lead to some changes in the reported results. Extension of this work to investigate the effect of source pressure amplitude on harmonics generation with varying temperature will be the topic of next project in this study.

\subsection{Acknowledgements}

The authors wish to acknowledge technical assistance from Arthur Worthington from Dept. of Physics, Ryerson University, Toronto, Canada. This work was partially supported by the Ontario Ministry of Research and Innovation Research Fund- Research Excellence (ORF-RE) grant, and the Natural Sciences and Engineering Research Council of Canada (NSERC Discovery grants) that were awarded to J. Tavakkoli and M. C. Kolios. Funding to purchase the equipment was provided by the Canada Foundation for Innovation, Canada Research Chairs program, and Ryerson University. 


\section{Chapter 3: Temperature dependence of acoustic harmonics in a lossy liquid: measurement and simulation ${ }^{2}$}

\subsection{Abstract}

The effect of temperature on acoustic harmonics generation in a mixture of $90 \%$ glycerol and $10 \%$ water (by volume), as a tissue-mimicking attenuating liquid, was studied over a temperature range of 26 to $46^{\circ} \mathrm{C}$. A temperature dependent Khokhlov-Zabolotskaya-Kuznetsov (KZK) model was used to simulate nonlinear acoustic wave propagation in the medium at temperatures of $26,31,36,41$ and $46^{\circ} \mathrm{C}$. For measurements, two single-element focused transducers were used to transmit tone bursts at $5 \mathrm{MHz}$ and $13 \mathrm{MHz}$ in the liquid at the same temperatures. A needle hydrophone was used to record the acoustic pressure along the axes of the transducers. The axial pressure profiles of the fundamental $\left(p_{1}\right)$, the second $\left(p_{2}\right)$ and the third $\left(p_{3}\right)$ harmonics where obtained in both simulations and measurements at each temperature and the change in their peak values were analyzed. For a temperature change from 26 to $46^{\circ} \mathrm{C}$, the values of $p_{1}, p_{2}, p_{3}, p_{2} / p_{1}$, and $p_{3} / p_{1}$ increased by $35 \%, 189 \%, 573 \%, 113 \%$ and $396 \%$, respectively at $5 \mathrm{MHz}$ in simulations. In experiments, the average values of $p_{1}, p_{2}, p_{3}, p_{2} / p_{1}$, and $p_{3} / p_{1}$ increased by $35 \%, 223 \%, 797 \%, 138 \%$ and $576 \%$, respectively. The harmonics were more sensitive to temperature at $13 \mathrm{MHz}$.

\footnotetext{
${ }^{2}$ This chapter is based on an original article submitted for publication as: B. Maraghechi, M. C. Kolios, J. Tavakkoli, "Temperature dependence of acoustic harmonics in a lossy liquid: measurement and simulation," The Journal of the Acoustical Society of America, Jan. 2016 (under review).
} 


\subsection{Introduction}

Thermal therapies are medical interventions that use heat to produce the desired clinical effect. Hyperthermia and thermal ablation are the two types of thermal therapies in which tissue temperature is increased to $39-43^{\circ} \mathrm{C}$ and above $55^{\circ} \mathrm{C}$, respectively [5]. A real-time temperature monitoring technique is needed to ensure that the target tissue temperature is achieved with minimal temperature increase elsewhere. Image-guided thermal therapy is clinically being performed with magnetic resonance imaging-guided focused ultrasound (MRgFUS) [38]. Ultrasound thermometry could potentially be an alternative to MRI since it can be done in real-time and it is inexpensive and portable.

The temperature dependence of acoustic parameters such as the speed of sound, the nonlinearity parameter (B/A), attenuation coefficient and backscattered coefficient has been studied in order to investigate the possibility of using these parameters for noninvasive temperature estimation using ultrasound [41, 38]. A number of studies have shown the dependence of the nonlinearity parameter, speed of sound and the attenuation coefficient on temperature for biological tissues in in vitro measurements $[58,50,61,60,51,53,89,90]$. Liu et al. presented the temperature dependence of B/A in ex vivo porcine fat and porcine liver samples and proposed the B/A as a basis for temperature estimation [53]. Sehgal et al. measured B/A as a function of temperature in human breast fat and excised normal and fatty human liver [89, 90]. Jackson et al. measured the B/A and the attenuation of ex vivo bovine liver samples in the temperature range of 23 to $50^{\circ} \mathrm{C}$ [51]. Choi et al. studied several acoustic parameters such as speed of sound, backscattered coefficient and attenuation coefficient of excised porcine liver tissue as a function of temperature [50]. In all the mentioned studies the speed of sound and B/A 
were reported to increase and the attenuation coefficient was reported to decrease with temperature up to $45-50^{\circ} \mathrm{C}$.

We have studied the temperature dependence of the pressure amplitude and the energy content of the backscattered fundamental frequency ( $p_{1}$ and $\left.E_{1}\right)$, the second $\left(p_{2}\right.$ and $\left.E_{2}\right)$ and the third ( $p_{3}$ and $E_{3}$ ) harmonics in tissue-mimicking gel phantoms and ex vivo bovine muscle tissues in pulse-echo mode at $13 \mathrm{MHz}$ [83]. In this study we found that the harmonics were increasing as a function of temperature with a high sensitivity in both phantoms and tissues.

Previous studies have reported the density, sound speed, B/A and attenuation coefficient of different liquids such as glycerol and water at various temperatures [54, 91, 92]. It was shown that the absorption coefficient has the highest sensitivity to temperature followed by B/A and then the speed of sound in both liquids.

van Dongen and Verweij studied the ratio of harmonics generated by nonlinear propagation in glycerol as a function of temperature using the lossless form of the Burgers' equation for acoustic plane waves [52]. They proposed a new noninvasive ultrasound thermometry technique based on the ratios between the fundamental frequency and the higher harmonics. In this technique reference harmonic ratios are obtained in a reference medium and for a given propagation distance over a range of temperatures. Unknown temperatures could then be estimated by comparing measured harmonic ratios with the corresponding reference ratios.

In this study, we report results of simulations and measurements of acoustic harmonics versus temperature in a mixture of glycerol and water, as an attenuating liquid, at frequencies of 5 and $13 \mathrm{MHz}$. The mixture was composed of 90\% (by volume) glycerol and 10\% (by volume) water (90/10 glycerol/water) in order to make a tissue-mimicking liquid in terms of its attenuation coefficient. A temperature dependent Khokhlov-Zabolotskaya-Kuznetsov (KZK) 
wave equation was used to simulate finite-amplitude ultrasound wave propagating in the liquid at temperatures from $26^{\circ} \mathrm{C}$ to $46^{\circ} \mathrm{C}$. A needle hydrophone was used to measure the acoustic pressure fields created by two single-element focused transducers in the liquid within the same temperature range. The axial harmonic profiles were obtained and analyzed at each temperature for both frequencies in both simulations and measurements.

\subsection{Materials and Methods}

\subsubsection{Simulations}

As the temperature increases in the medium, the attenuation coefficient, sound speed, B/A and the density change. All these parameters affect the wave propagation through the medium. Therefore, the simulations were performed using a modified KZK nonlinear wave equation in which temperature dependence of the medium parameters was included represented as [74]:

$$
\frac{\partial P}{\partial \sigma}=\frac{1}{4 G(T)} \int_{-\infty}^{\tau}\left(\frac{b}{a} \frac{\partial^{2} P}{\partial X^{2}}+\frac{a}{b} \frac{\partial^{2} P}{\partial Y^{2}}\right) \cdot d \tau^{\prime}+A(T) \frac{\partial^{2} P}{\partial \tau^{2}}+N(T)\left(P \frac{\partial P}{\partial \tau}\right),
$$

where the $G(T), A(T)$ and $N(T)$ are the linear focusing gain, and the absorption and nonlinear parameters defined as:

$$
G(T)=\frac{z_{0}(T)}{d}, \quad A(T)=\alpha_{0}(T) d, \quad N(T)=\frac{d}{\bar{z}},
$$

where $z_{0}=\omega_{0} a b / 2 c_{0}(T)$ is the Rayleigh distance at angular frequency $\omega_{0}$ for a source with characteristic sizes of $a$ and $b$ in the $x$ and $y$ directions. $X$ and $Y$ are dimensionless transverse coordinates with respect to $a$ and $b . \bar{z}=\rho_{0}(T) c_{0}^{3}(T) / \beta(T) \omega_{0} p_{0}$ is the plane wave shock formation distance where $\beta=1+B / 2 A$ is the coefficient of nonlinearity. $\sigma=z / d$ is the dimensionless $z$ axis. $P=\left(p / p_{0}\right)$ is the transformed source pressure amplitude where $p$ is the 
sound pressure in the Cartesian coordinates and $p_{0}$ is the source pressure amplitude. $\alpha_{0}$ is the pressure absorption coefficient, $d$ is the focal length, $T$ is the temperature, $c_{0}$ and $\rho_{0}$ are the small signal speed of sound and the medium density, respectively. $\tau=\omega_{0} t^{\prime}$ is the dimensionless retarded time which $t^{\prime}$ is the retarded time $\left(t^{\prime}=t-z / c_{0}\right)$ [74].

The source pressure waveform was defined as:

$$
P=\exp \left[-\left(\frac{\tau}{14 \pi}\right)^{2}\right] \sin \tau
$$

The empirical values of sound speed, B/A and the density of glycerol and water as a function of temperature where multiplied by their percentage concentrations in the mixture $(90 \%$ glycerol and $10 \%$ water) and added together $[54,92,52,85]$. The empirical values of attenuation coefficient in a 90/10 glycerol/water mixture (by volume) as a function of temperature were taken from Darbari et al. ${ }^{14}$ The temperature-dependent parameters used in the simulations are shown in Figure 3-1 [91].

Source parameters were set to $f_{0}=5 \mathrm{MHz}, a=3 \mathrm{~mm}, d=180 \mathrm{~mm}$, and $p_{0}=1.3 \mathrm{MPa}$ for the low frequency and $f_{0}=13 \mathrm{MHz}, a=3.55 \mathrm{~mm}, d=13 \mathrm{~mm}$, and $p_{0}=1 \mathrm{MPa}$ for the high frequency. Using the acoustic parameters of liquid at $26^{\circ} \mathrm{C}$ given in Figure 3-1 resulted in the nonlinearity and absorption parameters of $N=5.33$ and $A=5.37$ at $5 \mathrm{MHz}$ and $N=0.77$ and $A$ $=2.62$ at $13 \mathrm{MHz}$. The source pressures were chosen in order to achieve similar degree of nonlinearity as to that obtained in the experiment at the baseline temperature of $26^{\circ} \mathrm{C}$.

Pressure fields of the fundamental $\left(p_{1}\right)$, the second $\left(p_{2}\right)$ and the third $\left(p_{3}\right)$ harmonics where simulated at each temperature from 26 to $46^{\circ} \mathrm{C}$, in increments of $5^{\circ} \mathrm{C}$. Harmonics were then spatially averaged over a circular area corresponding to virtual area of the hydrophone used in the experiments to take into account the spatial averaging effect of the hydrophone $[87,86]$. 

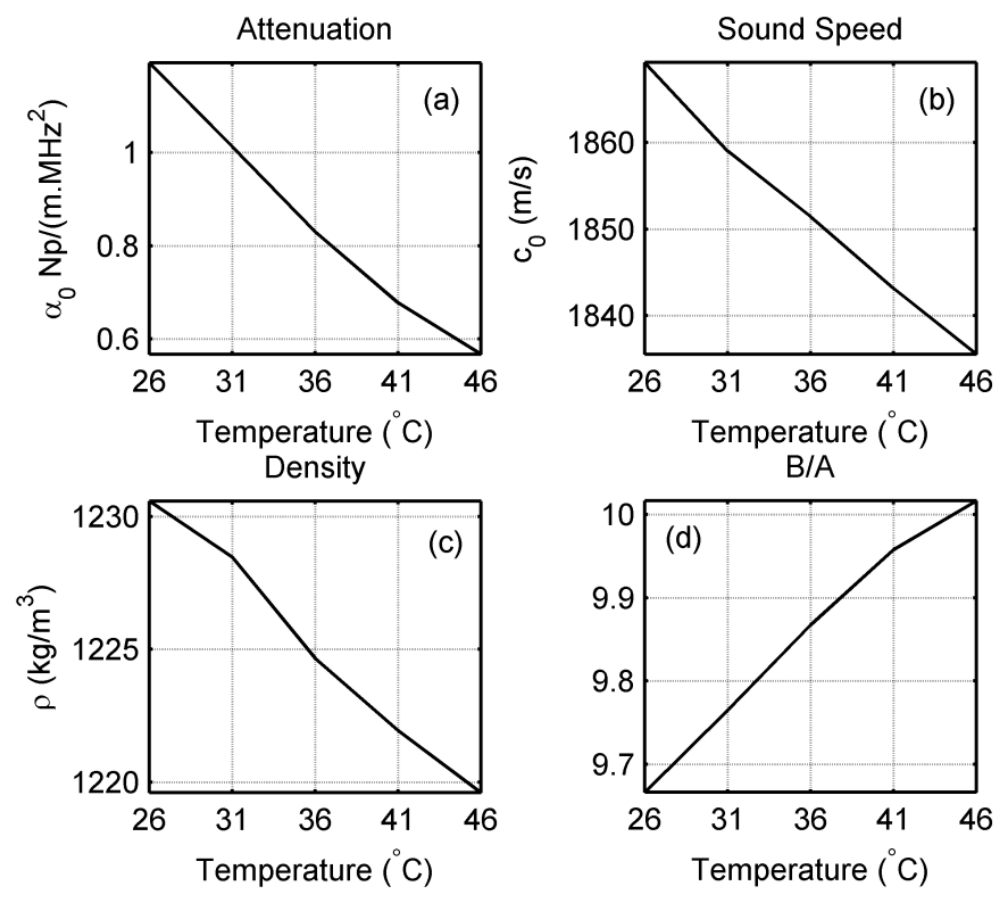

Figure 3-1: The values of (a) attenuation coefficient [91], (b) speed of sound [54, 92], (c) density [92, 52] and (d) B/A $[92,85]$ as a function of temperature in a 90/10 mixture of glycerol and water.

\subsubsection{Experiments}

The experiments at $5 \mathrm{MHz}$ were performed with a $6 \mathrm{~mm}$ diameter PZT transducer (Matec, Northborough, MA) with a center frequency of $5 \mathrm{MHz}$ and a focal length of $21 \mathrm{~cm}$. The transducer was driven with an arbitrary function generator (Model AFG3101; Tektronix, Beaverton, OR, USA) emitting 25-cycle long tone bursts at $5 \mathrm{MHz}$. The pulses were amplified with a $55 \mathrm{~dB}$ RF power amplifier (Model A150; E\&I, Rochester, NY, USA). The experiments at $13 \mathrm{MHz}$ were conducted using an ultrasound scanner (Vevo® 770, Visualsonics Inc., Toronto, ON, Canada) with a $25 \mathrm{MHz}$ center frequency wide-band single-element transducer (RMV710B, $7.1 \mathrm{~mm}$ diameter and $15 \mathrm{~mm}$ focal length).

Ultrasound signals were measured using a needle hydrophone with an active element diameter of $400 \mu \mathrm{m}$ (Model HNA-0400; ONDA Corporation, Sunnyvale, CA, USA) attached to 
a three-axis micro-positioning system. The detected signal was recorded with a $350 \mathrm{MHz}$ digital oscilloscope (Model 7032A; Agilent Technologies, Santa Clara, CA, USA). At each location, 128 waveforms were averaged in order to increase the signal-to-noise ratio.

The attenuating tissue-mimicking liquid was placed in a $2.5 \mathrm{~cm}$ diameter cylindrical container that was sealed from the bottom with an acoustic transparent window made of a thin sheet of Nitrile (Showa Best Glove, Inc., Menlo, GA). A pulsatile pump (Masterflex® L/S®, Cole Parmer, Chicago, IL) was used to pump hot water from a controlled circulating water bath (Haake DC10, Thermo Electron Corp., Newington, NH) around the container to increase the liquid temperature from 26 to $46^{\circ} \mathrm{C}$ in increments of $5^{\circ} \mathrm{C}$. The temperature of the liquid was monitored by a home-made needle thermocouple and a digital thermometer (Omegaette HH306, Omega Engineering Inc., Stamford, CT). A schematic diagram of the experimental arrangement is shown in Figure 3-2.

Pressure signals were recorded axially along the axis of symmetry of the transducers at each temperature. The pressure amplitudes of the fundamental frequency, the second and the third harmonics were obtained by calculating the frequency spectrum of the measured signals. The experiments were repeated six times and the average and standard deviation of the harmonics were computed at each location axially. 


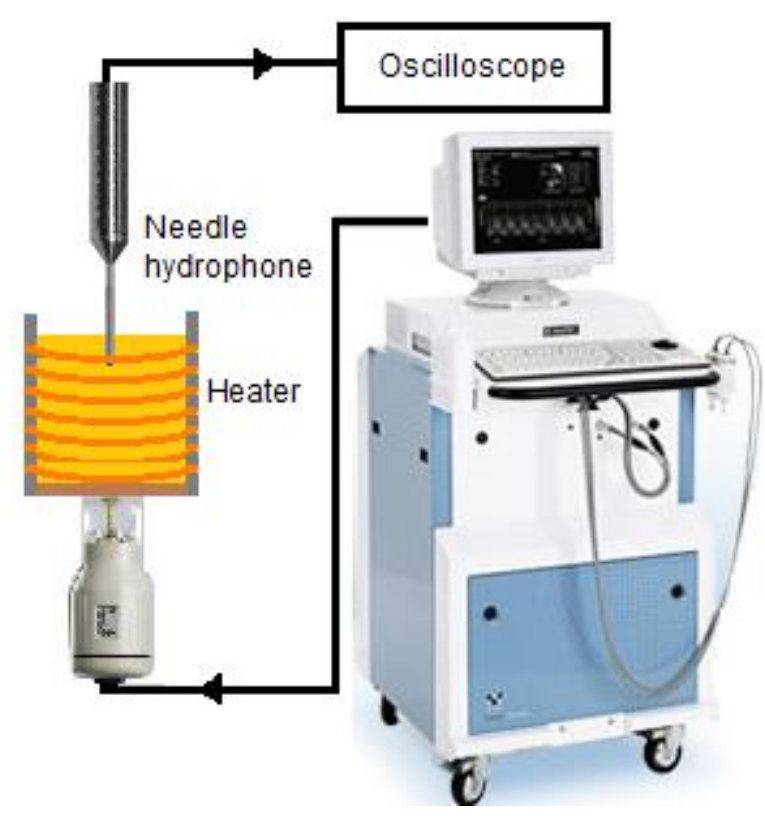

Figure 3-2: Schematic of the experimental setup at $13 \mathrm{MHz}$.

\subsection{Results}

The measured and simulated normalized pressure amplitudes of the first three harmonics along the acoustic axes of the transducers with transmit frequencies of $5 \mathrm{MHz}$ and $13 \mathrm{MHz}$ with respect to the maximum temperature $\left(46^{\circ} \mathrm{C}\right)$ at temperatures of $26,31,36,41$ and $46^{\circ} \mathrm{C}$ are shown in Figure 3-3 and Figure 3-4, respectively. The average and standard deviation of the change in peak values of the harmonics and their ratios as a function of temperature were calculated and are shown in Figure 3-5 and Figure 3-6. It should be noted that the third harmonic at $13 \mathrm{MHz}$ for the temperatures of $26^{\circ} \mathrm{C}$ and $31^{\circ} \mathrm{C}$ are not presented because of the low SNR in the measurements, mainly due to the high liquid attenuation. 

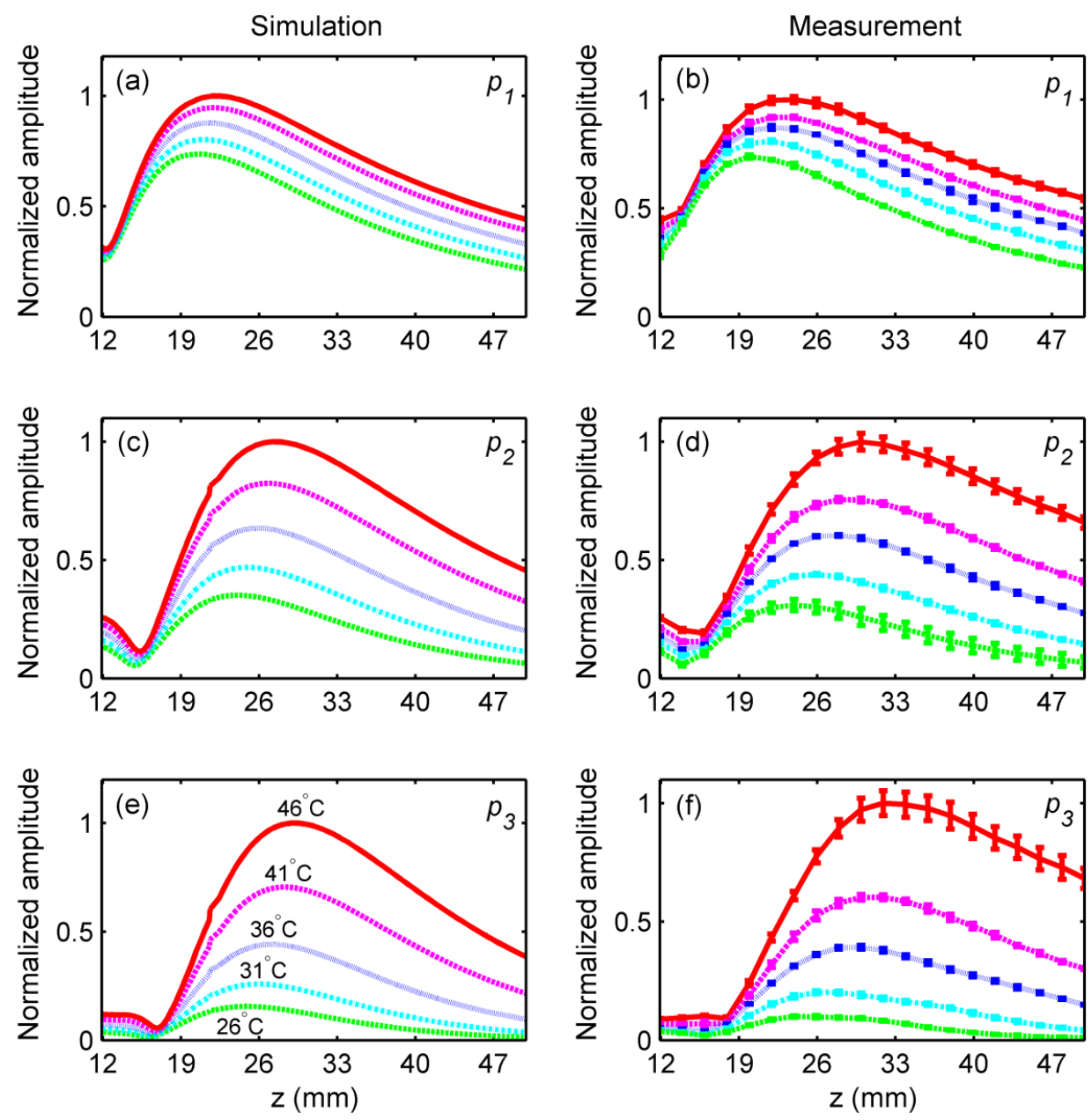

Figure 3-3: Simulated (left column) and measured (right column) normalized axial profiles of (a) and (b) $p_{1}$, (c) and (d) $p_{2}$, (e) and (f) $p_{3}$ as a function of temperature with respect to the maximum temperature $\left(46^{\circ} \mathrm{C}\right)$ at $5 \mathrm{MHz}$. The error bars represent the standard deviation of 6 independent trials. The correspondence between plots and temperatures is shown in panel (e). The magnitude of the harmonics increases monotonically with temperature from $26^{\circ} \mathrm{C}$ to $46^{\circ} \mathrm{C}$. 

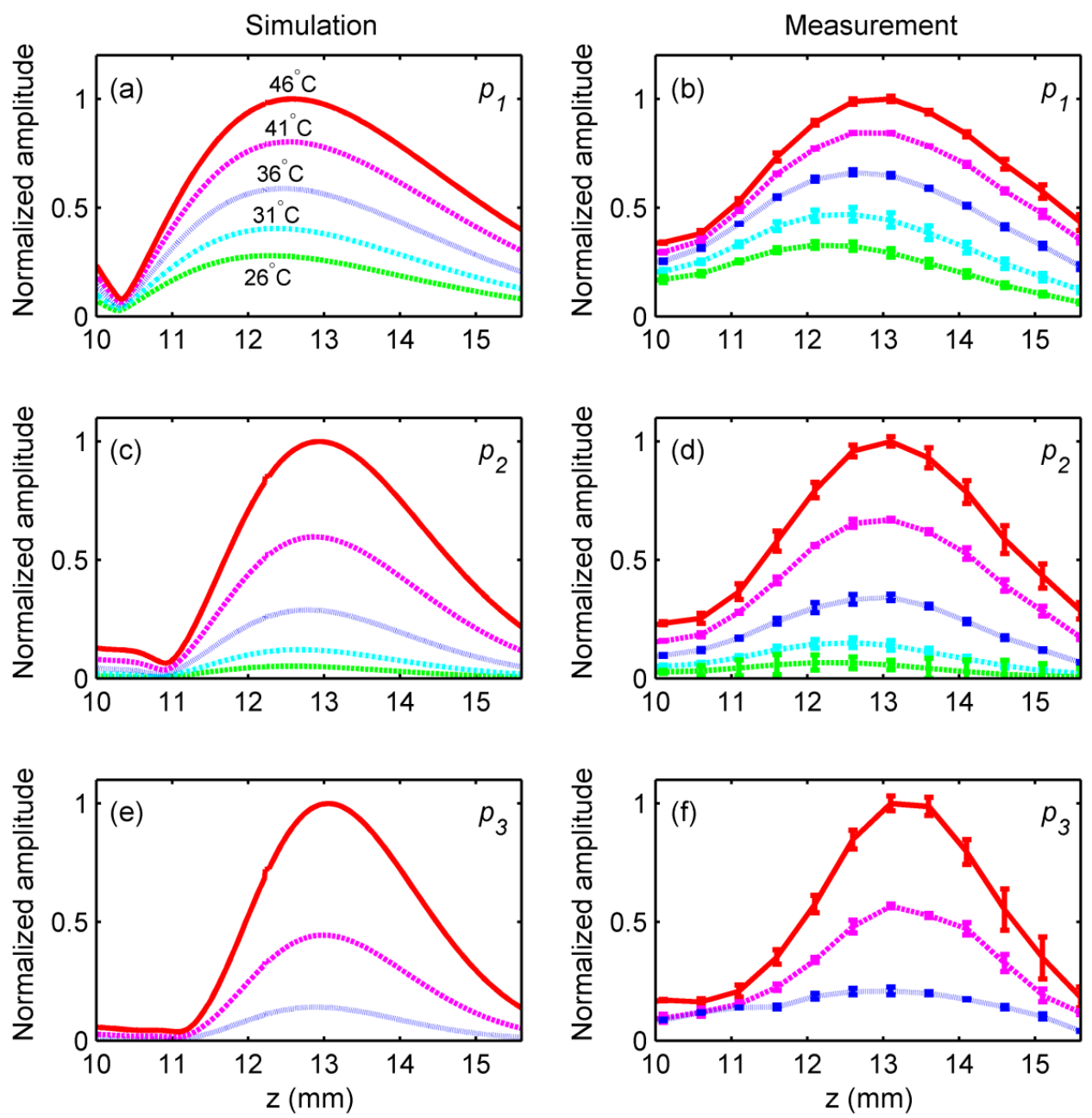

Figure 3-4: Simulated (left column) and measured (right column) normalized axial profiles of (a) and (b) $p_{1}$, (c) and (d) $p_{2}$, (e) and (f) $p_{3}$ as a function of temperature with respect to the maximum temperature $\left(46^{\circ} \mathrm{C}\right)$ at $13 \mathrm{MHz}$. The error bars represent the standard deviation of 6 independent trials. The correspondence between plots and temperatures is shown in panel (a). 

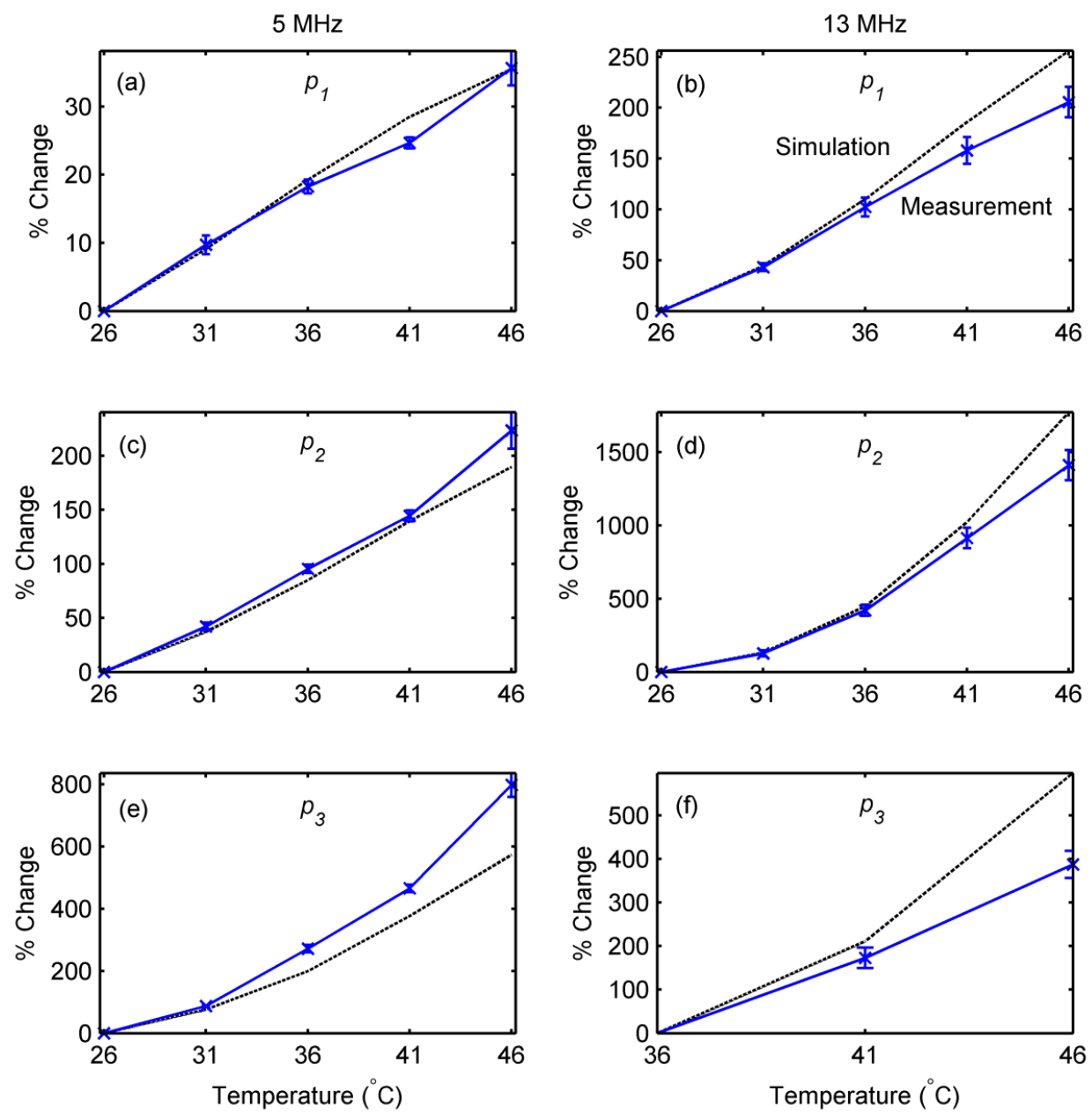

Figure 3-5: Simulated (dotted) and measured (solid) changes in the peak values of (a) and (b) $p_{1}$, (c) and (d) $p_{2}$, (e) and (f) $p_{3}$ as a function of temperature in a $90 / 10$ glycerol/water mixture at $5 \mathrm{MHz}$ (left column) and $13 \mathrm{MHz}$ (right column). The error bars represent the standard deviation of six trials. 

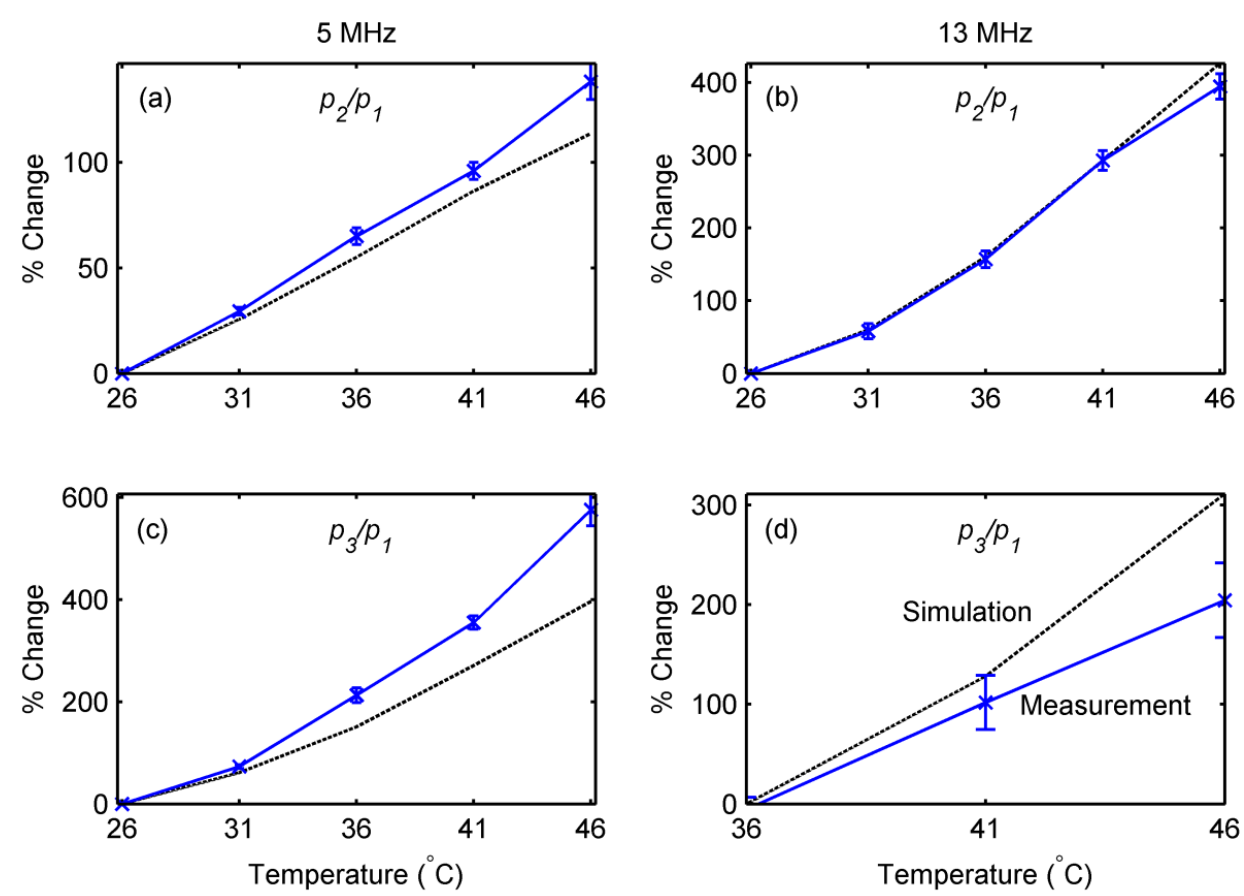

Figure 3-6: Simulated (dotted) and measured (solid) changes in the peak values of (a) and (b) $p_{2} / p_{1}$, and (c) and (d) $p_{3} / p_{1}$ as a function of temperature in a $90 / 10$ glycerol/water mixture at $5 \mathrm{MHz}$ (left column) and $13 \mathrm{MHz}$ (left column). The error bars represent the standard deviation of six trials.

\subsection{Discussion}

The plots of axial profiles (Figure 3-4 and Figure 3-5) show that the overall agreement between the measurements and the simulations is good at $26^{\circ} \mathrm{C}$ for both frequencies. However, some discrepancies occur at higher temperatures particularly at $5 \mathrm{MHz}$ transmit frequency. The largest mismatch is at $46^{\circ} \mathrm{C}$ for the third harmonic at $5 \mathrm{MHz}$. The drop in the harmonics ( $p_{2}$ and $\left.p_{3}\right)$ after the peak is steeper in simulations. This could be due incorrect values of the attenuation coefficient that used as input in the simulations.

The results obtained from simulations and measurements (Figure 3-3 to Figure 3-6) show that increase in temperature significantly enhances the creation of harmonics in a 90/10 mixture of glycerol and water. The nonlinear parameter $(N)$, the absorption parameter $(A)$ and the focusing gain $(G)$ were analyzed as a function of temperature in order to explain this effect. The 
$N$ and the $A$ parameters provide a measure of the relative importance of nonlinearity and absorption, respectively. The $G$ parameter is the peak value of $p / p_{0}$ at the geometric focus in the absence of both mechanisms of absorption and nonlinearity [84]. These parameters were defined in Eqs. 3- 2. Figure 3-7 shows the percentage change of the nonlinear and absorption parameters and the focusing gain versus temperature at frequencies 5 and $13 \mathrm{MHz}$. The nonlinear parameter and the focusing increase by $9.7 \%$ and $1.8 \%$, respectively and the absorption parameter decreases by $-52 \%$. The decrease in $A$ is only due to the reduction in attenuation and the increase in $G$ is only due to the decrease in sound speed $(-1.8 \%)$ as the liquid temperature increases. The increase in $N$ is due to the combined effects of increase in the acoustic nonlinearity parameter (3.62\%) and reduction in both the density (-0.9\%) and the speed of sound with temperature. A decrease in absorption and an increase in nonlinearity and focusing enhance the generation of harmonics as the temperature elevates from $26^{\circ} \mathrm{C}$ to $46^{\circ} \mathrm{C}$.
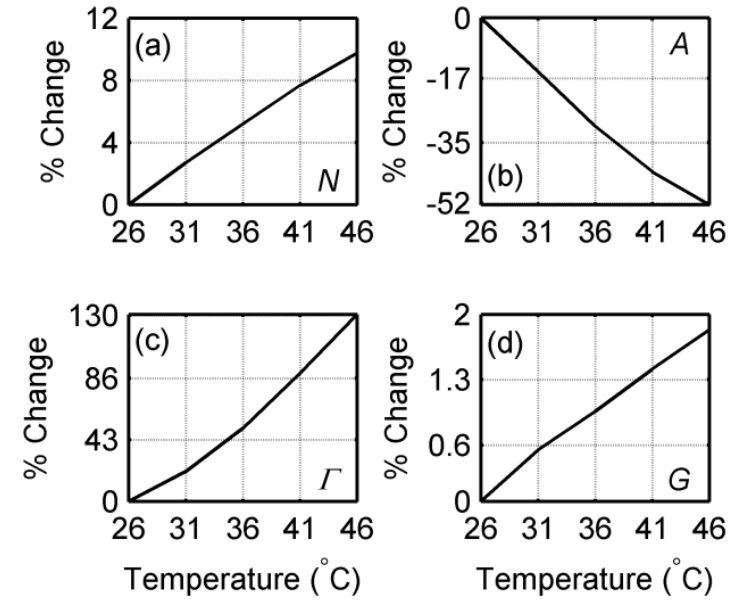

Figure 3-7: The percentage changes of (a) the nonlinear parameter $(N)$, (b) the absorption parameter $(A)$, (c) the Gol'dberg number $(\Gamma)$, and (c) the focusing gain $(G)$ as a function of temperature in a $90 / 10$ glycerol/water mixture at frequencies 5 and $13 \mathrm{MHz}$. 
The ratio of the two parameters is defined as the Gol'dberg number given as $\Gamma=N / A$ which shows the balance between the nonlinear and the absorption mechanisms [73]. The absorption process dominates when $\Gamma<1$ and the effects of nonlinearity and absorption are comparable when $\Gamma$ is close to 1.0 [73]. At the baseline temperature $\left(26^{\circ} \mathrm{C}\right)$ the Gol'dberg number is equal 0.99 and 0.29 at frequencies 5 and $13 \mathrm{MHz}$, respectively. Therefore, the effect of absorption is more pronounced at $13 \mathrm{MHz}$ and since the attenuation coefficient shows the highest sensitivity to temperature $(-52 \%)$, the temperature dependence of the harmonics is larger at $13 \mathrm{MHz}$ compared to that at $5 \mathrm{MHz}$.

Figure 3-3 to Figure 3-6 also show that higher harmonics have higher sensitivity to temperature. As we discussed in our previous work, this effect could be due to the fact that the pressure amplitude of $\mathrm{n}^{\text {th }}$ harmonic is proportional to $\left[\beta(T) /\left(\alpha_{0}(T) \rho_{0}(T) c_{0}{ }^{3}(T)\right)\right]^{n-1}$ for plane waves propagating in a lossy medium [83] [71]. This shows that higher harmonics have higher dependence to the acoustic parameters of the medium. Therefore, they will be more affected by the variations in the acoustic parameters due to temperature change.

Figure 3-3 and Figure 3-4 show that the acoustic focal positions have been shifted away from the transducer as the temperature elevated from $26^{\circ} \mathrm{C}$ to $46^{\circ} \mathrm{C}$. This could be due to the drop in the attenuation coefficient with temperature. However, this shift is smaller for the high frequency transducer (Figure 3-4) because it has a higher focusing gain compared to the lower frequency one.

The discrepancy between measurements and simulations in magnitudes of the changes in harmonics with temperature could be due to inaccurate input of acoustic parameters of the liquid as a function of temperature in the simulation model. The mismatch between measurements and simulations is more notable at the higher frequency and higher harmonics. A small difference in 
attenuation coefficient at $13 \mathrm{MHz}$ leads to large variations at 26 and $39 \mathrm{MHz}$ because of quadratic frequency dependence of attenuation in glycerol. Measurement errors in scanning the acoustic field, nonuniform distribution of temperature in the liquid, and variation in the hydrophone frequency response with temperature could have also contributed to this mismatch.

The increasing trend of the harmonics and their ratios with temperature at $13 \mathrm{MHz}$ is consistent with our previous results in water, tissue-mimicking gel phantoms and ex vivo bovine muscle at the same transmit frequency [83].

The harmonics could potentially be used as a basis for noninvasive thermometry due to their high sensitivity to temperature compared to other acoustic parameters such as sound speed and B/A. A next step in this study would be to investigate the feasibility of using the harmonics to estimate temperature in more realistic media such as tissue-mimicking gel phantoms and ex vivo tissue samples where only a localized target volume inside the medium is heated.

\subsection{Conclusion}

The harmonic generation in a mixture of $90 \%$ glycerol and $10 \%$ water, as an attenuating tissue-mimicking liquid, was studied as a function of temperature at two frequencies of 5 and 13 $\mathrm{MHz}$ in measurements and simulations. Simulations were conducted using the temperature dependent KZK nonlinear ultrasound wave propagation model and the experiments were performed with two single-element transducers. The results show that the harmonics have a strong temperature dependence in the liquid due to the combined effects of an increase in the nonlinear parameter $N$ and the focusing gain $G$, and a decrease in the absorption parameter $A$. Between the two frequencies studied, a higher sensitivity to temperature was observed at 13 MHz. A similar trend of increased harmonics generation with temperature was observed in 
tissue-mimicking gel phantoms and ex vivo bovine muscle at a $13 \mathrm{MHz}$ transmit frequency. The overall agreement between the measurements and the simulations was good.

\subsection{Acknowledgements}

The authors wish to acknowledge technical assistance from Arthur Worthington from Dept. of Physics, Ryerson University, Toronto, Canada. This work was partially supported by the Ontario Ministry of Research and Innovation Research Fund- Research Excellence (ORF- RE) grant, and the Natural Sciences and Engineering Research Council of Canada (NSERC Discovery grants) that were awarded to J. Tavakkoli and M. C. Kolios. Funding to purchase the equipment was provided by the Canada Foundation for Innovation, Canada Research Chairs program, and Ryerson University. 


\section{Chapter 4: Temperature dependence of acoustic harmonics generated by nonlinear ultrasound beam propagation in ex vivo tissue and tissue-mimicking phantoms ${ }^{3}$}

\subsection{Abstract}

Hyperthermia is a cancer treatment technique that could be delivered as a stand-alone modality or in conjunction with chemotherapy or radiation therapy. Noninvasive and real time temperature monitoring of the heated tissue improves the efficacy and safety of the treatment. A temperature-sensitive acoustic parameter is required for ultrasound-based thermometry. In this paper, the amplitude and the energy of the acoustic harmonics of ultrasound backscattered signal are proposed as suitable parameters for noninvasive ultrasound thermometry. A commercial high frequency ultrasound imaging system was used to generate and detect acoustic harmonics in tissue-mimicking gel phantoms and ex vivo bovine muscle tissues. The pressure amplitude and the energy content of the backscattered fundamental frequency $\left(p_{1}\right.$ and $\left.E_{1}\right)$, the second ( $p_{2}$ and $\left.E_{2}\right)$ and the third $\left(p_{3}\right.$ and $\left.E_{3}\right)$ harmonics generated by nonlinear ultrasound propagation were detected in pulse-echo mode. Temperature was increased from $26^{\circ} \mathrm{C}$ to $46^{\circ} \mathrm{C}$ uniformly through both samples. The amplitude and the energy content of the harmonics and their ratio were measured and analyzed as a function of temperature. The average $p_{1}, p_{2}$ and $p_{3}$ increased by $69 \%, 100 \%$ and $283 \%$, respectively as the temperature was elevated from $26^{\circ} \mathrm{C}$ to $46^{\circ} \mathrm{C}$ in tissue

\footnotetext{
${ }^{3}$ This chapter is based on an original article published as: B. Maraghechi, M. C. Kolios, J. Tavakkoli, “Temperature dependence of acoustic harmonics generated by nonlinear ultrasound beam propagation in ex vivo tissue and tissuemimicking phantom," International Journal of Hyperthermia, vol. 31, no. 6, pp. 666-673, Jul. 2015
} 
samples. In the same experiment, the average $E_{1}, E_{2}$ and $E_{3}$ increased by $163 \%, 281 \%$ and $2257 \%$, respectively. A similar trend was observed in tissue-mimicking gel phantoms. The findings suggest that the harmonics generated due to nonlinear ultrasound beam propagation are highly sensitive to temperature and could potentially be used for noninvasive ultrasound tissue thermometry.

\subsection{Introduction}

Hyperthermia is a cancer treatment method in which the tissue temperature is increased to $40-45^{\circ} \mathrm{C}$. Heat can destroy cancer cells or sensitize them to radiation and chemotherapeutic drugs [3]. Heat can also be used to cause the release of drugs from thermosensitive liposomes [6, 93]. Thermosensitive liposomes are being developed to release the drug within the hyperthermia temperature range of $39-43^{\circ} \mathrm{C}$, while remaining stable in non-tumor bloodstream at physiological temperature $\left(37^{\circ} \mathrm{C}\right)[5]$. This reduces the exposure of healthy organs to chemotherapy and lowers systemic toxicity $[6,5]$.

The second type of thermal therapy is thermal ablation which directly kills cells by inducing coagulation necrosis in tissue through elevating the temperature above $55^{\circ} \mathrm{C}$. Hyperthermia and thermal ablation are clinically approved for cancer treatment $[3,31,94]$.

There are a number of heating approaches available for thermal therapy based on ultrasound, microwave, radio frequency and infra-red (IR) laser energy deposition. Ultrasound has several advantages compared to other heating modalities due to its increased penetration depth in soft tissues and high degree of beam direction control. Moreover, ultrasound energy can be applied noninvasively to tissue [31, 94].

One of the therapeutic ultrasound devices in clinics uses high intensity focused ultrasound (HIFU) which deposits the energy of the beam into a small focal region placed in the 
region of interest in tissue. In a typical HIFU treatment, the temperature of the focal region can be increased to above $55^{\circ} \mathrm{C}$ in a few seconds which leads to rapid and highly localized coagulation necrosis in $[31,94]$.

Tissue heterogeneity and perfusion cause non-uniformities in heating the tumor tissue [3]. The other major limitation of thermal therapies is the lack of an accessible and reliable noninvasive and real time tissue temperature monitoring technique to control the treatment. Monitoring and controlling the tissue temperature distribution during thermal therapy will help to maintain the target temperature in the region of interest without affecting surrounding tissue, which improves the treatment efficacy and safety.

Magnetic resonance imaging (MRI) is considered as the gold standard modality for noninvasive temperature measurements in clinics [40]. Magnetic resonance imaging-guided focused ultrasound (MRgFUS) is the latest technology that has combined the anatomical details and 3-D temperature monitoring abilities of MRI and the therapeutic capability of focused ultrasound (FUS) [43, 44]. However, MRI thermometry is an expensive modality and suffers from low temporal resolution. Moreover, the heating applicator assembly must be MR compatible $[40,43]$.

Ultrasound is a non-ionizing, inexpensive, real time and portable modality which might be a superior alternative to other types of thermometry systems. Previously, three major ultrasonic methods have been proposed to measure the temperature noninvasively, based on estimations of: 1) the backscattered RF echo-shift [67], 2) the variation in the attenuation coefficient [57], and 3) the change in backscattered signal energy [62]. However, to date, none of these techniques have been adopted for clinical usage due to their lack of accuracy and robustness for in vivo noninvasive thermometry. 
The backscattered RF echo-shift technique, which has been shown to be the most promising ultrasound-based thermometry method, is based on two physical phenomena: 1) the change in the speed of sound with temperature and 2) thermal expansion of the medium due to heating [67]. However, there are several challenges that face noninvasive thermometry based on this technique [41, 68]. A major challenge for in vivo applications is related to tissue motion which includes respiratory and cardiac motions. Tissue motion produces mechanical shifts which are equivalent to temporal shifts and appear as a significant source of artifact in temperature maps [95].

Amongst all other acoustic parameters, such as the speed of sound and attenuation coefficient, the acoustic nonlinearity parameter (B/A) has also been shown to be temperature dependent $[89,90]$. Some investigators have proposed B/A as a basis for temperature estimation $[53,52]$. van Dongen and Verweij studied the temperature dependence of absorption coefficient, B/A, speed of sound and thermal expansion of water [52]. They showed that the absorption coefficient is the most temperature sensitive ultrasound physical parameter and B/A is the second most. The temperature dependence of $\mathrm{B} / \mathrm{A}$ in water was about four times greater than the temperature dependence of the speed of sound and thermal expansion for the same temperature range.

A relatively high-pressure acoustic wave (also known as finite-amplitude wave) becomes progressively distorted as it propagates, generating higher harmonics which can be detected in the signal frequency spectrum. For a given excitation and propagation distance, the extent of the pressure wave distortion and generation of the harmonics mainly depend on the B/A parameter, attenuation coefficient and the sound speed of the medium [71]. Since all three are sensitive to temperature changes, the acoustic harmonics are also expected to be temperature dependent [52]. 
In a numerical simulation study, van Dongen and Verweij showed changes in the amplitudes of the harmonics of a transmitted acoustic wave in glycerol as a function of temperature [52]. They demonstrated that noninvasive thermometry using the ratio of the fundamental over the second harmonic amplitudes is feasible, with an accuracy of $\pm 2{ }^{\circ} \mathrm{C}$.

In this study, the temperature sensitivity of harmonic amplitudes and their energy content generated by nonlinear ultrasound beam propagation in tissue is examined. Higher harmonics were generated and detected with a commercial high frequency ultrasound system used for small animal imaging. The measurements were performed in pulse echo mode on tissue-mimicking gel phantoms and ex vivo bovine muscle tissue samples. The temperature sensitivity of the harmonics in both samples is presented. In this work, we demonstrate that the acoustic harmonics are highly temperature dependent and could potentially be considered as a suitable parameter for noninvasive ultrasound thermometry.

\subsection{Materials and Methods}

A high-frequency ultrasound imaging scanner (Vevo® 770, Visualsonics Inc., Toronto, ON, Canada) with a $25-\mathrm{MHz}$ center frequency wide-band (100\% fractional bandwidth) singleelement transducer (RMV-710B, f-number 2.1, $15 \mathrm{~mm}$ focal length) was used to generate and detect acoustic harmonics. Mechanical sector sweep of the single element transducer produced 2D RF frames. Higher harmonics were generated through transmitting a 13-MHz 30-cycle pulse that resulted in a focal peak pressure of approximately $3.9 \mathrm{MPa}$ at the baseline temperature of $26^{\circ} \mathrm{C}$ in water, measured by a calibrated hydrophone with $0.2 \mathrm{~mm}$ active element diameter (HPM02/1, Precision Acoustics, Dorchester, UK).

The pressure amplitudes of the fundamental frequency $\left(p_{1}\right)$, the second $\left(p_{2}\right)$ and the third $\left(p_{3}\right)$ harmonics were obtained from the frequency spectra of the backscattered radiofrequency 
(RF) signals from tissue-mimicking gel phantoms and ex vivo bovine muscle tissues. The backscattered energy of the RF signal $(E)$, the fundamental frequency $\left(E_{1}\right)$, the second $\left(E_{2}\right)$ and the third $\left(E_{3}\right)$ harmonics were also calculated for both media.

The gel phantom was composed of $90.4 \%$ distilled and degased water (by weight), $7.8 \%$ w/v gelatin, $1 \%$ w/v polyethylene oxide and $0.8 \%$ w/y formaldehyde. Freshly excised ex vivo bovine muscle tissues were obtained from a local butcher shop. The tissue samples were cut to a size of around $3 \mathrm{~cm} \times 8 \mathrm{~cm} \times 2 \mathrm{~cm}$ and immersed in $0.9 \%$ degassed saline solution at $5^{\circ} \mathrm{C}$ for 12 hours prior to each experiment. Before the experiment, the muscle specimens were degassed in a desiccator connected to a vacuum pump (2581, Welch, Monroe, LA) for 30 minutes in order to remove large air pockets from the tissue.

Both the sample and the transducer were immersed in a temperature controlled circulating water bath (Haake DC10, Thermo Electron Corp., Newington, NH) with a temperature accuracy of $\pm 0.1^{\circ} \mathrm{C}$ in order to induce a homogeneous heat distribution throughout the sample. A schematic of the experimental setup is shown in Figure 4-1. The experiments on $e x$ vivo tissue samples were performed in a phosphate buffered saline (PBS) solution. The temperature was increased from $26^{\circ} \mathrm{C}$ to $46^{\circ} \mathrm{C}$ for both samples. In order to reach the steady state, the sample was kept 30 minutes at each temperature before making the measurement. The transducer was kept outside the water bath (and at room temperature) in between measurements to minimize the time duration when the transducer is exposed to higher temperatures. The transducer was immersed into the water bath only after the sample had reached its equilibrium temperature.

Two home-made calibrated needle thermocouples were inserted in the sample, and the temperature was recorded using a digital thermometer (Omegaette HH306, Omega Engineering 
Inc., Stamford, CT). Before data collection at each temperature, the water circulator was turned off in order to eliminate vibrations. Care was taken to remove air bubbles on the surface of the sample and the transducer. The experiments on tissue-mimicking gel phantoms and ex vivo tissue samples were repeated in different samples 5 and 6 times, respectively.

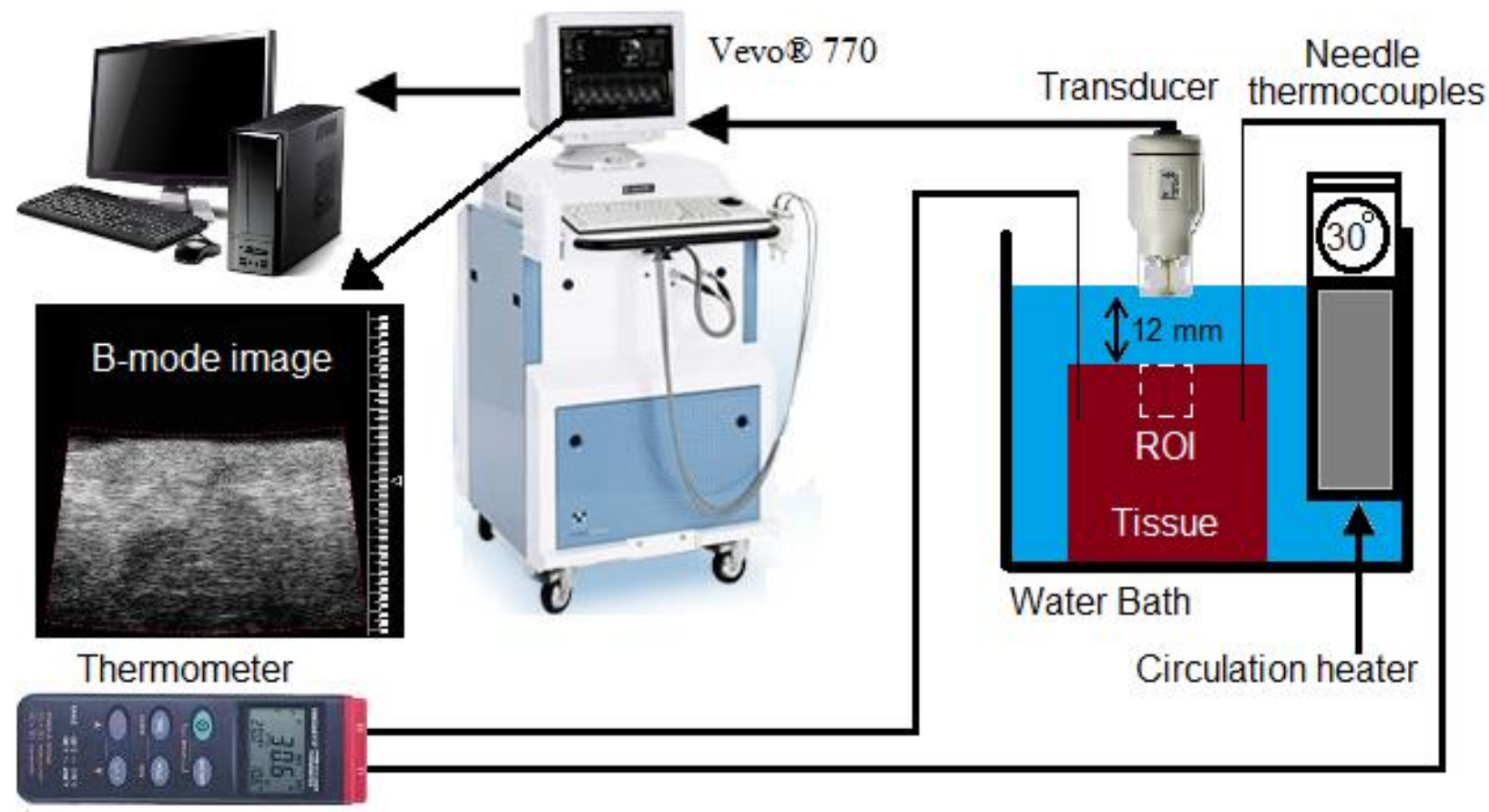

Figure 4-1: Schematic of the experimental setup.

RF echo data collection was performed when the samples reached the steady state temperature. At each temperature (from $26^{\circ} \mathrm{C}$ to $46^{\circ} \mathrm{C}$ ), 50 consecutive backscattered $\mathrm{RF}$ frames were captured for averaging with a frame rate of $1 \mathrm{~Hz}$. Each frame consisted of $100 \mathrm{RF}$ backscattered echo lines covering a region of interest $(\mathrm{ROI})$ of $11 \times 16 \mathrm{~mm}^{2}$ within the sample beginning from its surface. The RF signal sampling rate was $420 \mathrm{MSa} / \mathrm{s}$.

A set of experiments on ex vivo bovine muscle tissues were also performed in order to investigate the effect of tissue coagulation and possible bubble formation on the harmonics during the 4-hour course of the experiment, particularly during the time when the temperatures 
were at their highest values. The experiments were performed similar to the ones described above except that the heating process consisted of two steps. First, the temperature of the tissue samples was increased from $26^{\circ} \mathrm{C}$ to $44^{\circ} \mathrm{C}$ in 40 minutes and second, they were kept at $44^{\circ} \mathrm{C}$ for 200 minutes. RF data collection was performed every 10 minutes after the tissue was heated to $44^{\circ} \mathrm{C}$ (between 40 to 240 minutes timestamps).

\subsubsection{Data analysis}

The acquired RF data frames were analyzed offline using algorithms developed in MATLAB (MathWorks Inc., Natick, MA). Two methods were used to assess the temperature dependence of the generated harmonics: 1) the peak value of the backscattered pressure harmonics and their ratios $\left(p_{1}, p_{2}, p_{3}, p_{2} / p_{1}\right.$ and $\left.p_{3} / p_{1}\right)$, and 2$)$ the backscattered energy of the RF signal $(E)$, of the harmonics and their ratios $\left(E_{1}, E_{2}, E_{3}, E_{2} / E_{1}\right.$ and $\left.E_{3} / E_{1}\right)$.

For the first technique, the harmonics were obtained by calculating the frequency spectrum of the windowed backscatter signal. The window size for taking the Fourier transform was chosen $10 \times \tau(\sim 0.77 \mu \mathrm{s})$ to obtain the $p_{1}, p_{2}$ and $p_{3}$ of the echo signal, where $\tau$ is the period of the fundamental frequency $(13 \mathrm{MHz})$. Gaussian windows were used with step size of $10 \times \tau$ with no overlap between two consecutive windows. The frequency spectrum of each windowed signal along the RF data line was first averaged over the 50 frames and then averaged with the windows at the same depth along all the $100 \mathrm{RF}$ lines (spanning a range of $11 \mathrm{~mm}$ ). The pressure harmonic amplitudes and their ratios $\left(p_{1}, p_{2}, p_{3}, p_{2} / p_{1}\right.$ and $\left.p_{3} / p_{1}\right)$ were calculated and plotted for

each window. The maximum values were then recorded and analyzed as a function of temperature. 
For the second technique, the backscattered energy of the RF signal and the harmonics in the focal region with an area of $5 \times 16 \mathrm{~mm}^{2}$ was calculated. The ROI was limited to the focal region in order to improve the signal to noise ratio. The backscattered energies of the harmonics were obtained by first filtering the RF signal using the Filter Design and Analysis Tool (FDATool) in the MATLAB Signal Processing Toolbox. Three band pass equiripple Finite Impulse Response (FIR) filters were designed to pass frequencies between $9 \mathrm{MHz}$ to $16 \mathrm{MHz}, 23$ $\mathrm{MHz}$ to $29 \mathrm{MHz}$ and $36 \mathrm{MHz}$ to $42 \mathrm{MHz}$ in order to separate the fundamental frequency, the second and the third harmonics content of the RF signal, respectively. All three filters had an order of 100. Equiripple FIR filter has equal ripples in both passband and stopband by minimizing the maximum approximation error between the desired and designed filter responses. This results in reduction of a large ripple at the edge of the passband at the cost of increasing the transition band [96].

The envelope of the signal was found using Hilbert transform. Envelope values were squared to calculate the backscattered energy at each data point on the RF signal [63]. The energies were summed over the region of interest for each frame. The energy ratios of $E_{2} / E_{1}$ and $E_{3} / E_{1}$ were obtained by dividing the summed energy of the second and the third harmonics over that of the fundamental frequency. Averaged backscattered energy was calculated using 50 frames recorded at each temperature.

\subsection{Results}

Figure 4-2 shows the averaged (100 RF lines) peak pressure values of the a) fundamental frequency, b) second, and c) third harmonics in the gel phantom and tissue sample at temperatures of $26^{\circ} \mathrm{C}, 34^{\circ} \mathrm{C}, 38^{\circ} \mathrm{C}$ and $46^{\circ} \mathrm{C}$. The harmonics were primarily generated in the focal region and increased as the temperature was raised. 

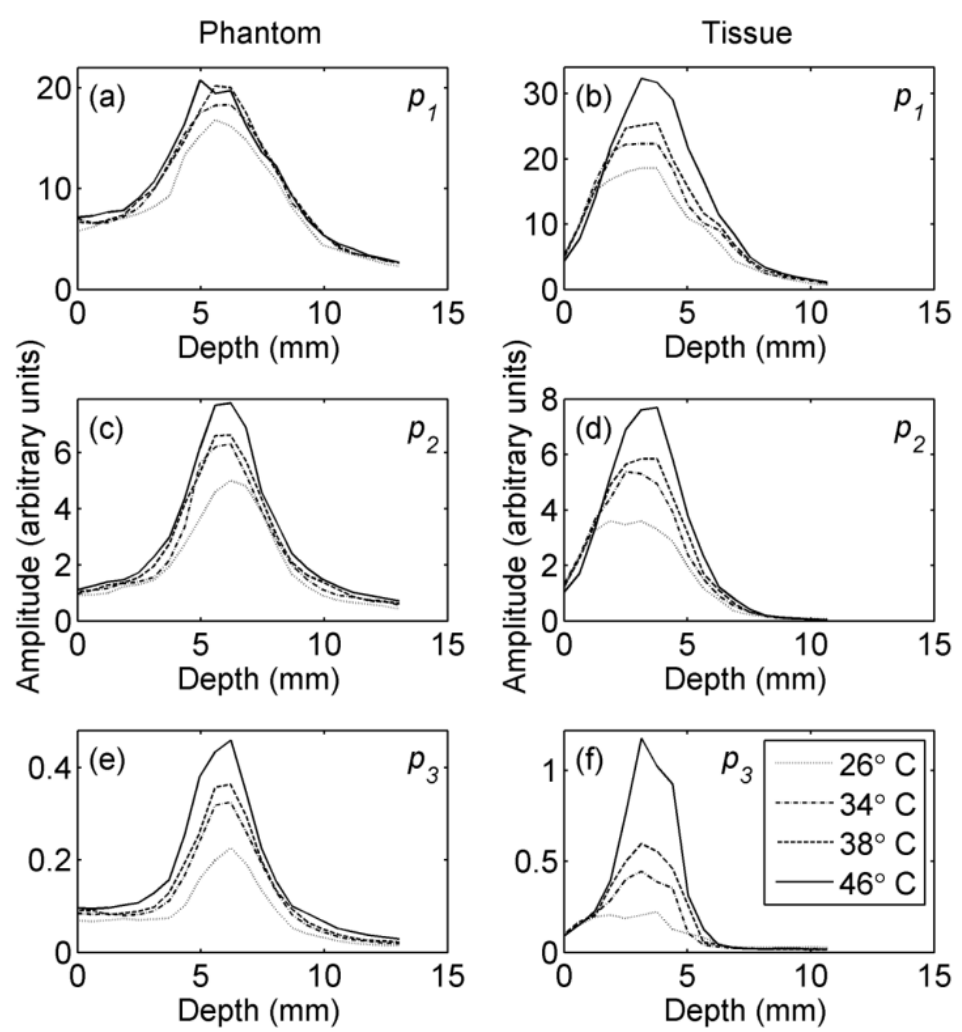

Figure 4-2: The averaged (100 RF lines) values of (a) and (b) $p_{1}$, (c) and (d) $p_{2}$, (e) and (f) $p_{3}$ in tissue-mimicking gel phantom (left column) and ex vivo bovine muscle tissue (right column) at temperatures of $26^{\circ} \mathrm{C}, 34^{\circ} \mathrm{C}, 38^{\circ} \mathrm{C}$ and $46^{\circ} \mathrm{C}$.

Similar graphs were plotted for all data sets obtained from tissue and gel samples. The maximum value of the pressure harmonics at each temperature was chosen. The average and standard deviation of the maxima of the harmonics and their ratios were calculated for each temperature. Figure 4-3 and Figure 4-4 show the relative changes of the $p_{1}, p_{2}, p_{3}, p_{2} / p_{1}$ and $p_{3} / p_{1}$ as a function of temperature for tissue-mimicking gel phantoms and ex vivo tissue samples, respectively. The percentage increase in the harmonics and their ratio due to the $20^{\circ} \mathrm{C}$ temperature rise from $26^{\circ} \mathrm{C}$ to $46^{\circ} \mathrm{C}$ is summarized in Table 4-1 for both samples. 

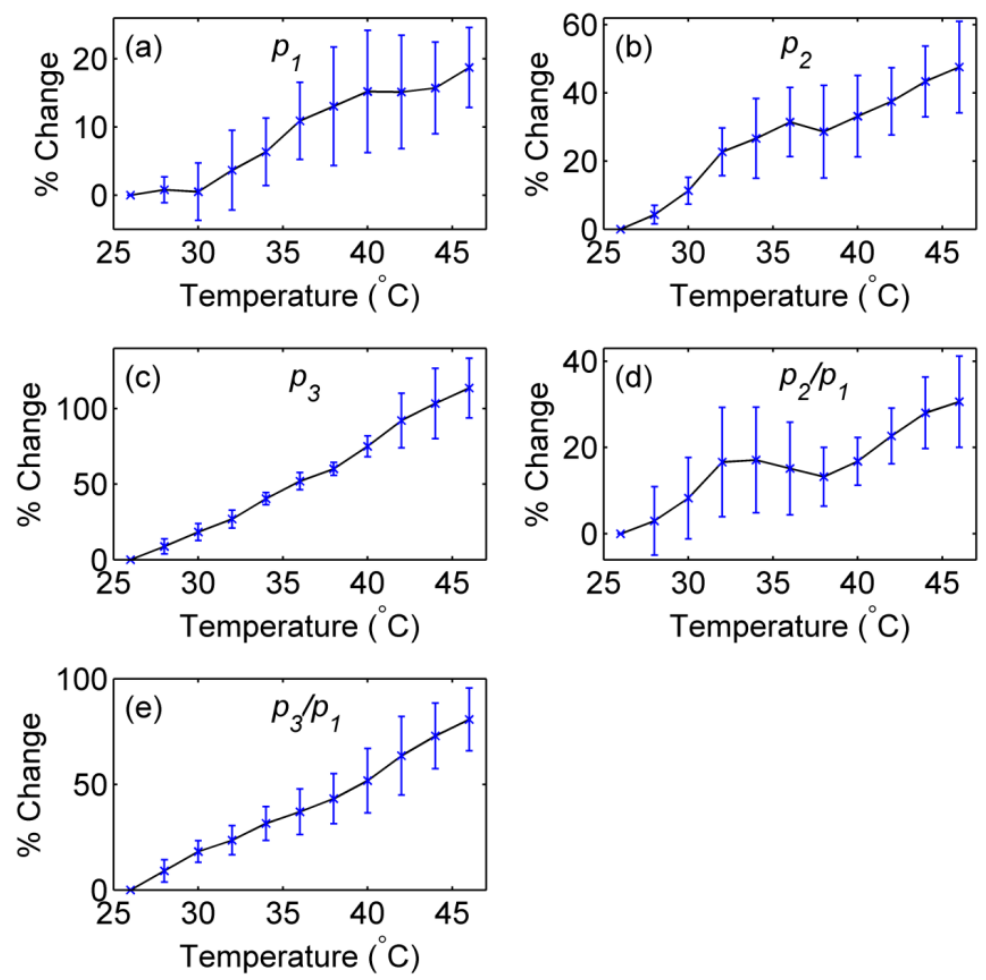

Figure 4-3: Changes in (a) $p_{1}$, (b) $p_{2}$, (c) $p_{3}$ and the ratios (d) $p_{2} / p_{1}$ and (e) $p_{3} / p_{1}$ as a function of temperature in tissue-mimicking gel phantoms with respect to the initial temperature $\left(26^{\circ} \mathrm{C}\right)$. The error bars represent the standard deviation of 5 trials. 

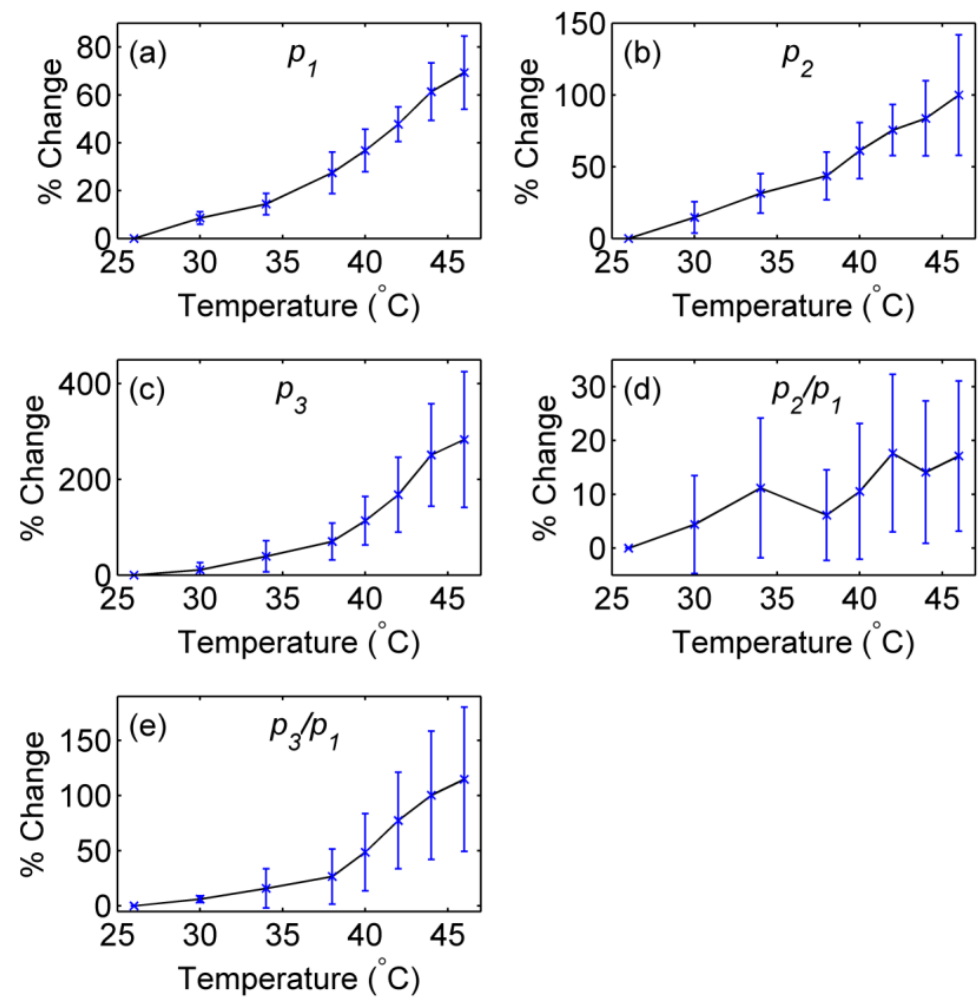

Figure 4-4: Changes in (a) $p_{1}$, (b) $p_{2}$, (c) $p_{3}$ and the ratios (d) $p_{2} / p_{1}$ and (e) $p_{3} / p_{1}$ as a function of temperature in $e x$ vivo bovine muscle tissues with respect to the initial temperature $\left(26^{\circ} \mathrm{C}\right)$. The error bars represent the standard deviation of 6 trials.

\begin{tabular}{ccc}
\hline $\begin{array}{c}\text { Harmonic } \\
\text { amplitude }\end{array}$ & $\begin{array}{c}\% \text { increase in gel } \\
\text { phantoms }\end{array}$ & $\begin{array}{c}\% \text { increase in } \\
\text { tissue samples }\end{array}$ \\
\hline$p_{1}$ & $18 \% \pm 6 \%$ & $69 \% \pm 15 \%$ \\
$p_{2}$ & $47 \% \pm 13 \%$ & $100 \% \pm 42 \%$ \\
$p_{3}$ & $113 \% \pm 19 \%$ & $283 \% \pm 141 \%$ \\
$p_{2} / p_{1}$ & $30 \% \pm 10 \%$ & $17 \% \pm 13 \%$ \\
$p_{3} / p_{1}$ & $80 \% \pm 14 \%$ & $114 \% \pm 65 \%$ \\
\hline
\end{tabular}

Table 4-1: Percentage increase in $p_{1}, p_{2}, p_{3}$ and the ratios $p_{2} / p_{1}$ and $p_{3} / p_{1}$ as the temperature was increased from $26^{\circ} \mathrm{C}$ to $46^{\circ} \mathrm{C}$ in tissue-mimicking gel phantoms and ex vivo tissue samples.

The average and standard deviation of the backscattered energies of the signal, of the harmonics and their ratios at each temperature were calculated. Figure 4-5 and Figure 4-6 show the relative changes of the $E, E_{1}, E_{2}, E_{3}, E_{2} / E_{1}$ and $E_{3} / E_{1}$ as a function of temperature for tissue- 
mimicking gel phantoms and ex vivo tissue samples, respectively. The percentage increase in the backscattered energy of the RF signal, of the harmonics and their ratios due to the $20^{\circ} \mathrm{C}$ temperature rise from $26^{\circ} \mathrm{C}$ to $46^{\circ} \mathrm{C}$ is summarized in Table 4-2 for both samples.
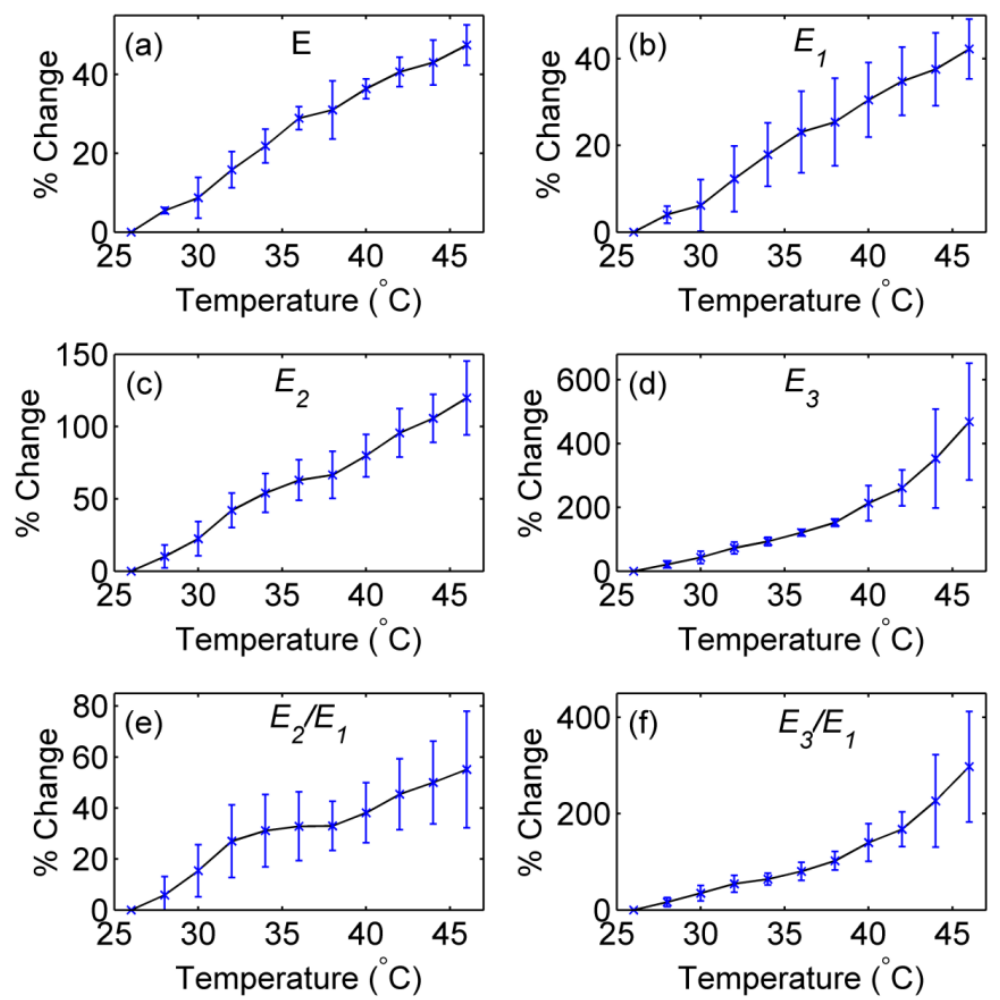

Figure 4-5: Changes in (a) $E$, (b) $E_{l}$, (c) $E_{2}$, (d) $E_{3}$ and the ratios (e) $E_{2} / E_{1}$ and (f) $E_{3} / E_{1}$ at the focal region as a function of temperature in tissue-mimicking gel phantoms with respect to the initial temperature $\left(26^{\circ} \mathrm{C}\right)$. The error bars represent the standard deviation of 5 trials. 

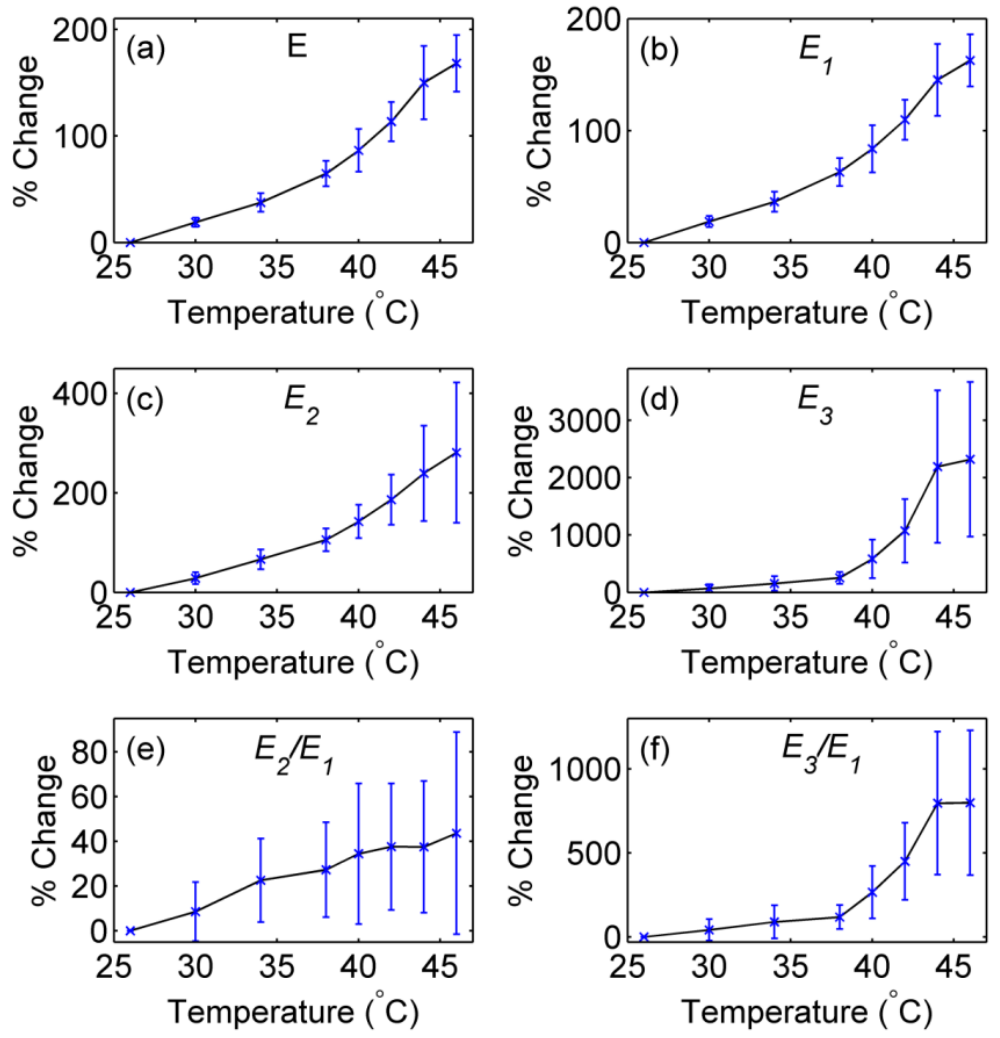

Figure 4-6: Changes in (a) $E$, (b) $E_{1}$, (c) $E_{2}$, (d) $E_{3}$ and the ratios (e) $E_{2} / E_{1}$ and (f) $E_{3} / E_{1}$ at the focal region as a function of temperature in ex vivo bovine muscle tissues with respect to the initial temperature $\left(26^{\circ} \mathrm{C}\right)$. The error bars represent the standard deviation of 6 trials.

\begin{tabular}{ccc}
\hline $\begin{array}{c}\text { Backscattered } \\
\text { harmonic energy }\end{array}$ & $\begin{array}{c}\% \text { increase in gel } \\
\text { phantoms }\end{array}$ & $\begin{array}{c}\% \text { increase in } \\
\text { tissue samples }\end{array}$ \\
\hline$E$ & $48 \% \pm 5 \%$ & $168 \% \pm 28 \%$ \\
$E_{1}$ & $42 \% \pm 7 \%$ & $163 \% \pm 24 \%$ \\
$E_{2}$ & $121 \% \pm 24 \%$ & $281 \% \pm 143 \%$ \\
$E_{3}$ & $470 \% \pm 198 \%$ & $2257 \% \pm 1394 \%$ \\
$E_{2} / E_{1}$ & $55 \% \pm 22 \%$ & $43 \% \pm 44 \%$ \\
$E_{3} / E_{1}$ & $297 \% \pm 123 \%$ & $770 \% \pm 444 \%$
\end{tabular}

Table 4-2: Percentage increase in $E, E_{1}, E_{2}, E_{3}$ and the ratios $E_{2} / E_{1}$ and $E_{3} / E_{1}$ as the temperature was increased from $26^{\circ} \mathrm{C}$ to $46^{\circ} \mathrm{C}$ in tissue-mimicking gel phantoms and ex vivo tissue samples. 
The average and standard deviation of the harmonic amplitudes and energies were calculated as a function of time while the samples were kept at $44^{\circ} \mathrm{C}$ temperature for 200 minutes. Figure 4-7 shows the relative changes of the $p_{1}, p_{2}, p_{3}, E_{1}, E_{2}$ and $E_{3}$ with time.
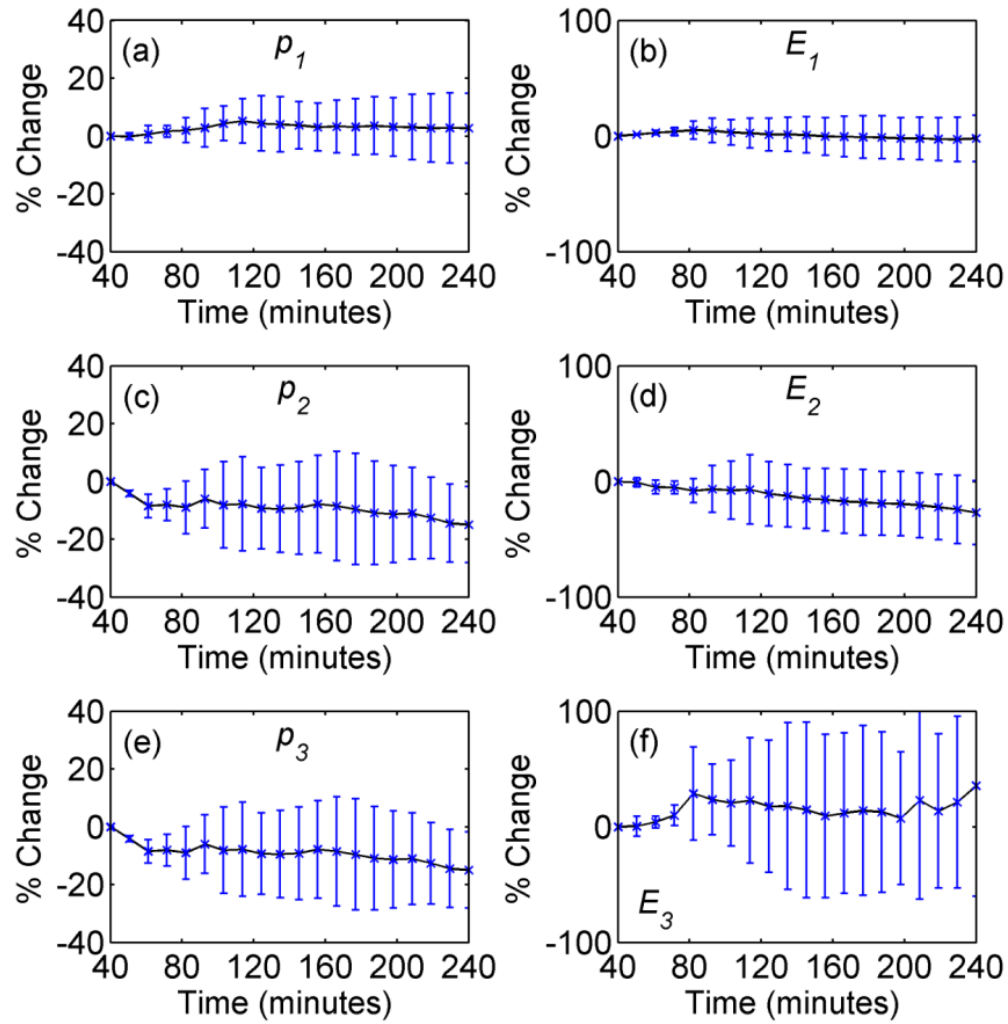

Figure 4-7: Changes in (a) $p_{1}$, (b) $E_{1}$, (c) $p_{2}$, (d) $E_{2}$, (e) $p_{3}$ and (f) $E_{3}$ at $44^{\circ} \mathrm{C}$ as a function of time in ex vivo bovine muscle tissues with respect to the initial time (40 minutes). The error bars represent the standard deviation of 4 trials.

\subsection{Discussion}

As the ultrasound beam propagates in the nonlinear regime, harmonics including the second and the third are generated and backscattered by the scatterers in the medium. Figure 4-2 shows the growth of harmonics in the focal region of the focused transducer which is due to the cumulative effect of nonlinear beam propagation and rise in acoustic pressure in the focal region. The figure also shows that temperature sensitivity of the nonlinear propagation is more significant for the higher harmonics. The shift in the position of the harmonics peak toward the 
transducer in the ex vivo bovine muscle tissue (right column of Figure 4-2) is because the tissue samples were placed further away from the transducer. Therefore, the focal region of the transducer (which is the region of creation of harmonics) is closer to the surface of the tissue. This was done in order to increase the signal to noise ratio of the harmonics, since the attenuation is significantly higher in tissue samples compared to the gel phantoms.

The amount of wave distortion and the amplitude of the higher harmonics detected using the backscattered signal depend on the attenuation coefficient, the parameter of nonlinearity (B/A), the sound speed and the density of the medium.

The Khokhlov-Zabolotskaya-Kuznetsov (KZK) equation is a well-established model for finite-amplitude beam propagation that consists of terms to account for diffraction, absorption and nonlinearity. The nonlinear term in the KZK equation contains $\beta /\left(\rho_{0} c_{0}^{3}\right)$, where $\beta$ is the coefficient of nonlinearity defined as $\beta=1+1 / 2(B / A), c_{0}$ is the speed of sound and $\rho_{0}$ is the medium density [71]. All three medium parameters vary with temperature [52].

It has been shown that the attenuation coefficient in most soft tissues decreases with temperature (up to $50^{\circ} \mathrm{C}$ ) and the slope of this decrease is steeper at higher frequencies $[58,61$, 60, 56]. Gammell et al. studied the temperature and frequency dependence of the attenuation coefficient in porcine liver, back fat and spleen and human liver [61]. In all the samples the attenuation coefficient decreased with temperature from $20^{\circ} \mathrm{C}$ to $37^{\circ} \mathrm{C}$ for the frequency range of 1.5-10 MHz. In the back fat tissue, the same trend was observed up to $49^{\circ} \mathrm{C}$. They also demonstrated that the rate of this drop in the attenuation coefficient increased with frequency. Similar results were also reported by Bamber and Hill for frequency range of 1-7 MHz in freshly excised tissues [58]. Ghoshal et al. showed that the attenuation coefficient decreased by $10 \%$ $20 \%$ with temperature from $37^{\circ} \mathrm{C}$ to $50^{\circ} \mathrm{C}$ in rabbit and beef liver [60]. 
Several studies have shown the dependence of the nonlinear parameter on temperature for biological tissues in in vitro measurements [89, 90, 53, 52]. Liu et al. presented the temperature dependence of $\mathrm{B} / \mathrm{A}$ for porcine fat and porcine liver [53]. They demonstrated that $\mathrm{B} / \mathrm{A}$ increased about $40 \%$ and $55 \%$ in porcine fat and liver, respectively as the temperature was raised from $20^{\circ} \mathrm{C}$ to $60^{\circ} \mathrm{C}$. Sehgal et al. showed that B/A in excised human liver increased by increasing the temperature from $20^{\circ} \mathrm{C}$ to $37^{\circ} \mathrm{C}[90]$.

The tissue-mimicking gel phantom used in this study was mostly composed of water $(90 \% \mathrm{w} / \mathrm{v})$. In water, the parameter of nonlinearity increases by about $7.3 \%$, and the attenuation coefficient decreases by about $-40 \%$ with temperature from $26^{\circ} \mathrm{C}$ to $46^{\circ} \mathrm{C}[52,54,85]$. It can therefore be expected that the changes of the acoustic parameters of the gel phantoms with temperature are similar to that in water $[97,98,99,100]$.

Figure 4-3 to Figure 4-6 show an increase of the harmonic amplitudes with temperature, and demonstrate that the higher the harmonic, the higher is its sensitivity to temperature. These could be due to the combined effects of temperature dependence of the attenuation coefficient, the coefficient of nonlinearity, the speed of sound, and the medium density. For a finiteamplitude plane wave propagating in an attenuating medium, the second and the third harmonics pressure amplitudes are proportional to $\beta /\left(\alpha \rho_{0} c_{0}{ }^{3}\right)$ and $\left[\beta /\left(\alpha \rho_{0} c_{0}{ }^{3}\right)\right]^{2}$, respectively [71]. Therefore, the temperature dependence of the acoustic parameters manifests themselves more prominently in the higher harmonics causing them to be more sensitive to temperature. The higher temperature dependence of the higher harmonics may also be explained by the frequency and temperature dependence of attenuation.

The results of Figure 4-3 and Figure 4-4 show that the increase in the acoustic harmonics with temperature in the tissue samples is about two to three times larger than that in the gel 
phantoms. The energy of harmonics increased by approximately three to four times more in tissue samples compared to that in the gel phantoms for comparable temperature change (shown in Figure 4-5 and Figure 4-6). This could be due to the different temperature dependence of the acoustic parameters in each medium. In the temperature range used in this study, the speed of sound increases by approximately $2 \%$ in the gel phantom but in the muscle tissue it increases by approximately $1.4 \%[97,101]$. The nonlinear term in the KZK equation shows that the increase in sound speed results in reduction in harmonics. Therefore, the smaller the increase in sound speed, the larger the increase in the harmonics.

The $p_{2} / p_{1}$ in Figure 4-3 and Figure 4-4 shows a relatively nonmonotonic increase as a function of temperature comparing to $p_{3} / p_{1}$. This can be because the relative increase in $p_{2}$ with temperature is larger than that of $p_{1}$ but not as significantly as compared to $p_{3}$.

As shown in Figure 4-4 and Figure 4-6, the standard deviation of the harmonic amplitudes increases as the temperature rises in the ex vivo bovine muscle tissues. This can be due to decomposition and protein denaturation in the tissue since it was kept in water bath for a long period of time (30 minutes at each of the temperatures which led to a total experiment time of about four hours for a given tissue sample). The thermal dose (TD), as a widely-accepted metric to measure the degree of soft tissue coagulation, was calculated for a point in the center of ROI [102]. The calculated TD was equal to 47 minutes at $43^{\circ} \mathrm{C}$ when the temperature was increased from $26^{\circ} \mathrm{C}$ to $44^{\circ} \mathrm{C}$. The TD was equal to 223 minutes at $43^{\circ} \mathrm{C}$ by including the last heating increment. Therefore, if one assumes a thermal dose threshold of 240 minutes at $43^{\circ} \mathrm{C}$ for coagulation necrosis in muscle tissue [103], some necrosis might have occurred mainly at the surface of the tissue sample during the last measurement while the temperature increased from $44^{\circ} \mathrm{C}$ to $46^{\circ} \mathrm{C}$. 
Figure 4-7 shows no significant change in the amplitudes and the energies of the harmonics while the tissue was kept at $44^{\circ} \mathrm{C}$ for 200 minutes. This shows that the increase in the harmonics amplitude and energy was mainly due to temperature changes and tissue coagulation or possible presence of bubbles has not confounded the measurements.

As mentioned earlier, to minimize the time duration of exposing the transducer to higher temperatures, it was kept outside the water bath in between measurements, and when immersed in water, the RF data collection was done in less than two minutes at each temperature. Moreover, to further investigate the possible effect of temperature variations of the transducer on the measurements, a separate bounce test was performed, in which a quartz plate reflector was placed at the transducer's focal point and the echo signal amplitude bounced back from the plate reflector was recorded by the transducer. The bounce test assembly was placed inside the water bath when the water temperature was increased from $26^{\circ} \mathrm{C}$ to $46^{\circ} \mathrm{C}$ in increments of $2^{\circ} \mathrm{C}$. It was observed that the measured peak to peak pressure amplitude was changed by less than only $\pm 0.5 \%$, that is insignificant comparing to our measurements uncertainties.

\subsection{Conclusion}

In this study, the temperature dependence of the acoustic harmonics generated by nonlinear ultrasound beam propagation was investigated in tissue-mimicking gel phantoms and ex vivo tissue samples in pulse echo mode using a commercial high-frequency ultrasound imaging scanner. The acoustic harmonics show high sensitivity to temperature compared to other acoustic parameters such as the speed of sound and the attenuation coefficient. The temperature dependence of the harmonic amplitudes and energies increase with frequency and it is most noticeable for the third harmonic. The average $p_{1}, p_{2}$ and $p_{3}$ increased by $69 \%, 100 \%$ and $283 \%$ when the temperature was increased from $26^{\circ} \mathrm{C}$ to $46^{\circ} \mathrm{C}$ in tissue samples. Whereas the change in 
speed of sound, which is the basis of the ultrasound echo-shift thermometry technique, for the same temperature range is about $1.5 \%$ in non-fatty soft tissues [68]. The results indicate that the acoustic harmonics generated by nonlinear ultrasound beam propagation could potentially be used for non-invasive temperature monitoring in tissue.

\subsection{Acknowledgements}

The authors wish to acknowledge the technical assistance of Arthur Worthington, Graham Pearson and Luke Yaraskavitch, all from Dept. of Physics, Ryerson University, Toronto, Canada. This work was partially supported by the Ontario Research Fund - Research Excellence (ORF-RE) grant and the Natural Sciences and Engineering Research Council of Canada (NSERC Discovery grants) that were awarded to J. Tavakkoli and M. C. Kolios. Funding to purchase the equipment was provided by the Canada Foundation for Innovation, the Ontario Ministry of Research and Innovation, and the Ryerson University and the Canada Research Chairs program. 


\section{Chapter 5: Feasibility of noninvasive temperature estimation using acoustic harmonics in tissue-mimicking gel phantoms ${ }^{4}$}

\subsection{Abstract}

A real-time noninvasive tissue temperature monitoring technique is required to estimate the temperature distribution during thermal therapy in order to monitor and control the treatment. The main objective of this study is to obtain 2D temperature maps in a tissue-mimicking gel phantom using the temperature dependence of acoustic harmonics. A peristaltic pump was used to circulate hot water through a stainless steel needle inserted in a tissue-mimicking gel phantom. Temperature was locally changed from $26^{\circ} \mathrm{C}$ to $46^{\circ} \mathrm{C}$ within the sample. Imaging was performed in pulse-echo mode using a commercial high-frequency ultrasound scanner. The change in the backscattered energy of the fundamental frequency, the second and the third harmonics were used to estimate the temperature changes. A conventional echo-shift technique was also implemented for comparison. Data collection was performed for two conditions to investigate the effect of motion on both techniques by: (1) inducing vibration in the phantom through the peristatic pump and, (2) subsequently with no phantom vibration while the pump was off. 2D temperature and echo-shift gradient maps where obtained in the gel phantom using the acoustic harmonics and the echo-shift techniques, respectively, with and without the presence of vibration in the sample. In the presence of vibration, noninvasive thermometry was feasible only by using the harmonics. The findings of this study suggest that analysis of the temperature dependence of

\footnotetext{
${ }^{4}$ This chapter is based on a manuscript under preparation as: B. Maraghechi, M. C. Kolios, J. Tavakkoli, "Feasibility of noninvasive temperature estimation using acoustic harmonics," International Journal of Hyperthermia, 2016 (under preparation).
} 
the acoustic harmonics can be used to develop a more robust noninvasive ultrasound-based thermometry technique to motion compared to the echo-shift method.

\subsection{Introduction}

Many researchers have been working to develop noninvasive tissue temperature monitoring methods [38]. The main motivation behind these studies is to monitor the temperature distribution in the region of interest during thermal therapy in order to uniformly heat the target tissue, (e.g. a tumor), while preserving the surrounding healthy tissue.

Invasive thermometry is being performed in clinics using thermocouples and fiber-optics sensors $[38,41]$. However, these devices are invasive and provide only single-point temperature measurements. Magnetic resonance imaging provides volumetric temperature measurement with relatively high spatial and temperature resolutions [40]. However, MRI systems are expensive and the heating modality has to be compatible with the strong magnetic fields of the imaging system. Ultrasound is an alternative and potentially attractive option for thermometry due to its characteristics such as being inexpensive, portable, non-ionizing and having a fast data acquisition and image processing capabilities [38, 41]. Several ultrasound based methods have been proposed for noninvasive temperature measurement such as the backscattered RF echo-shift technique, change in backscattered energy, and shear wave thermometry [38, 41].

Amongst all ultrasound based thermometry methods, the backscattered RF echo-shift technique is the most established method which is based on tracking the shift in echo signal due to change in speed of sound and thermal expansion [38,41]. Simon et al. previously showed that temperature change in a uniform medium could be estimated using [67]: 


$$
\delta T(z)=\frac{c_{0}}{2}\left(\frac{1}{\alpha-\lambda}\right) \frac{d}{d z}(\delta t(z))
$$

where $c_{0}$ is the speed of sound (SOS) at initial temperature, $\lambda$ is the thermal coefficient of the SOS, $\alpha$ is the linear coefficient of thermal expansion of the medium, and $\delta t(z)$ is the cumulative time-shift at depth z. The first term in Eq. 5- 1, $\frac{c_{0}}{2}\left(\frac{1}{\alpha-\lambda}\right)$, is a material-dependent parameter denoted by $k$, and the second term, $\frac{d}{d z}(\delta t(z))$, is the axial gradient of the cumulative shifts in the RF echo signal.

An important limitation of the backscattered RF echo-shift technique is its high sensitivity to motion (respiratory and cardiac motions) present in almost all in vivo situations. Mechanical shifts due to tissue motion can mask temporal shifts due to temperature change and appear as artifacts in temperature maps. Therefore, using this technique, robust temperature estimation has not been applied clinically. However, promising results have been shown in in vivo studies to reduce the sensitivity of this technique to motion [39, 104].

Previously, a noninvasive thermometry method was proposed based on the ratio of the acoustic harmonics generated by the nonlinear wave propagation [52]. Simulations were performed to study the temperature dependence of the harmonics in a lossless medium in transmit mode for a plane wave of finite amplitude [52]. Maraghechi et al. studied the temperature dependence of harmonic amplitudes and their ratios in water at several frequencies and showed that harmonics are monotonically increasing function of temperature at $13 \mathrm{MHz}$ [105].

Our group previously showed that the pressure amplitude and the backscattered energy of the fundamental frequency $\left(p_{1}\right.$ and $\left.B E_{1}\right)$, the second $\left(p_{2}\right.$ and $\left.B E_{2}\right)$ and the third $\left(p_{3}\right.$ and $\left.B E_{3}\right)$ harmonic in tissue-mimicking gel phantoms and ex vivo bovine muscle tissues are temperature 
dependent and that this temperature dependence could be considered as a basis for an ultrasoundbased method for noninvasive thermometry [83]. It was demonstrated that the average $B E_{1}, B E_{2}$ and $B E_{3}$ increased by $163 \%, 281 \%$ and $2257 \%$, respectively in tissue samples as the temperature was uniformly elevated from 26 to $46^{\circ} \mathrm{C}$. In gel phantoms, the average $B E_{1}, B E_{2}$ and $B E_{3}$ increased by $42 \%, 121 \%$ and $470 \%$, respectively in the same temperature range [83].

In the current study, the feasibility of obtaining 2D temperature change maps was investigated by estimating the change in backscattered energy of the acoustic harmonics and comparing it with the conventional RF echo-shift technique in a tissue-mimicking gel phantom for localized heating. The effect of medium motion on each method was also studied.

\subsection{Materials and Methods}

The experiments were performed on a tissue-mimicking gel phantom composed of distilled and degassed water $(90.4 \% \mathrm{w} / \mathrm{v})$, gelatin $(7.8 \% \mathrm{w} / \mathrm{v})$, polyethylene oxide $(1 \% \mathrm{w} / \mathrm{v})$ and formaldehyde $(0.8 \%$ w/y). A high-frequency ultrasound scanner (Vevo® 770, FujiFilm VisualSonics Inc., Toronto, ON, Canada) with a wide-band single-element transducer (RMV710B, 25-MHz center frequency, f-number 2.1, $15 \mathrm{~mm}$ focal length) was used to transmit pulse trains of 30-cycle length at $13 \mathrm{MHz}$. 2-D RF frames were produced by the mechanical sector sweep of the single element transducer.

The transducer was placed on top of the phantom and it was coupled to the phantom using an acoustic gel. A $1.6 \mathrm{~mm}$ thick stainless steel needle was inserted in the gel phantom and hot water, at various temperatures, was circulated in the needle in order to increase the temperature of the phantom only locally around the needle. Hot water was pumped in the needle from a temperature-controlled water bath (Haake DC10, Thermo Electron Corp., Newington, NH) using a peristaltic pump (Masterflex® L/S®, Cole Parmer, Chicago, IL). 
The needle was placed laterally $2 \mathrm{~mm}$ away from the imaging plane of the transducer in order to minimize the RF signal distortion from the presence of the needle. Figure 5-1 shows the schematic of the experimental setup and the needle position. A home-made calibrated needle type $\mathrm{K}$ thermocouple was inserted in the phantom, at the same depth as the heating needle within the imaging plane ( $2 \mathrm{~mm}$ away from the heating needle) and the temperature was recorded using a digital thermometer (Omegaette HH306, Omega Engineering Inc., Stamford, CT). The temperature at the thermocouple location in the imaging plane changed from $26^{\circ} \mathrm{C}$ to $46^{\circ} \mathrm{C}$ to examine how this would affect nonlinear propagation. This was accomplished by changing the temperature of the water flowing through the needle.

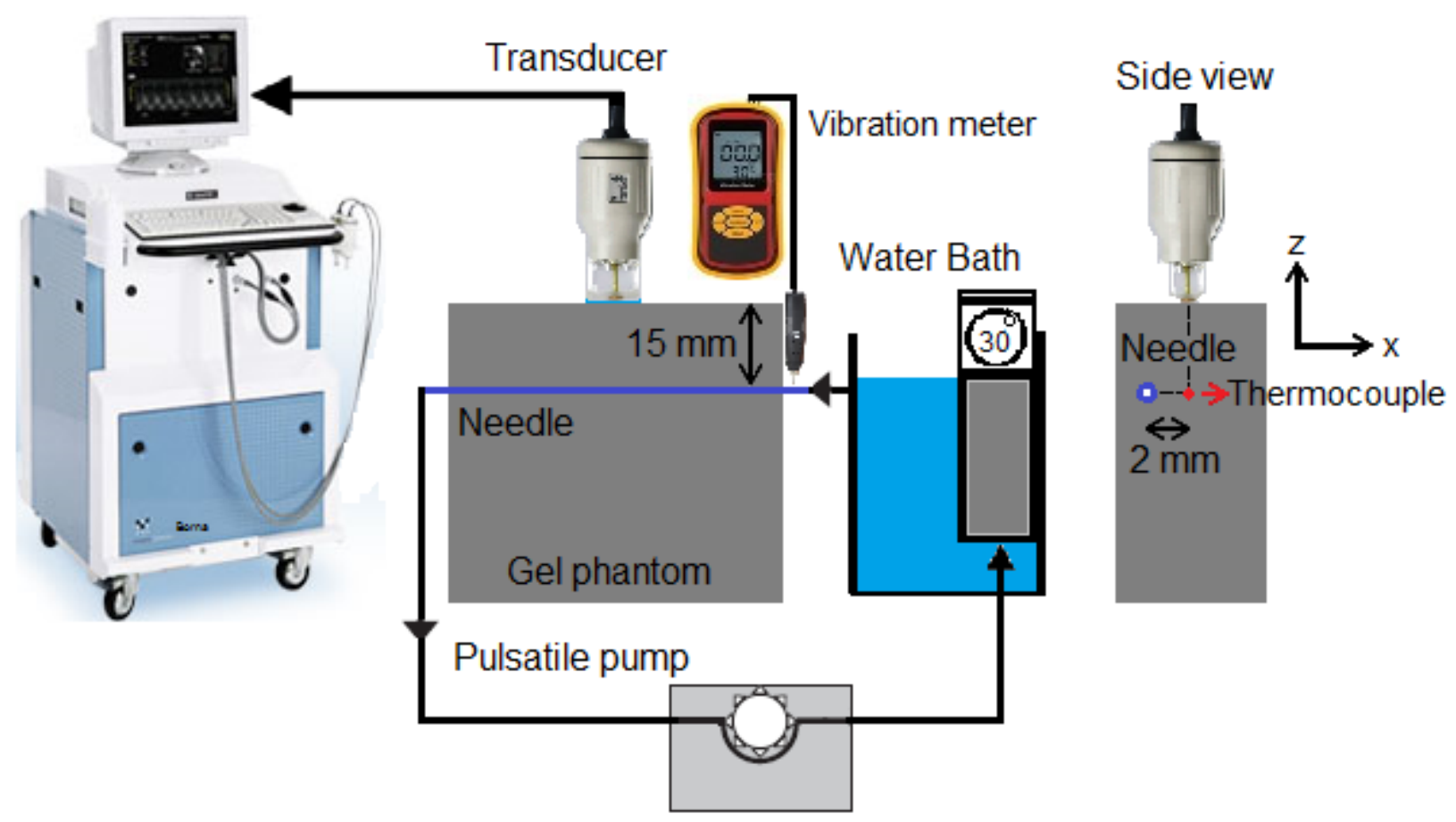

Figure 5-1: Schematic of the experimental setup.

In this study, two sets of experiments were performed. In the first set, data collection was performed while the temperature was increasing from 26 to $46^{\circ} \mathrm{C}$ by pumping hot water through the needle. The pump was also acted as a gentle source of motion to vibrate the phantom. Data 
collection was performed for the second time while the temperature was cooled down when the water bath and the peristaltic pump were turned off to eliminate vibrations in the phantom. The frequency of the induced vibration was estimated from the ultrasound M-mode data collected using the same transducer during the experiment. However, the M-mode data could not provide reliable estimation of displacement values along $x$ and $y$ directions as it is mostly sensitive to motion only in the ultrasound beam propagation direction ( $z$ direction). A calibrated vibration meter (GM63B, BENETECH, China) capable of measuring displacements in three dimensions within a range of 0.001-1.999 $\mathrm{mm}$, was used to measure the vibration displacement in the heating needle just before entering the gel phantom (shown in Figure 5-1) along $x, y$ and $z$ directions. The experiments were repeated 5 times in two different samples of the tissue-mimicking gel phantoms of the same composition for both set of experiments (with and without presence of vibration in the phantom).

The backscattered energy of the fundamental frequency, the second and the third harmonics were calculated by first, filtering the RF signal for each harmonic and then squaring the envelope values at each data point on the signal [83]. The change in the backscattered energy of each harmonic $\left(C B E_{i}\right)$ at each temperature $(j)$ was obtained by:

$$
C B E_{i}(j)=\frac{B E_{i}(j)-B E_{i}\left(26^{\circ} \mathrm{C}\right)}{B E_{i}\left(26^{\circ} \mathrm{C}\right)+\overline{B E_{l}\left(26^{\circ} \mathrm{C}\right)}} \times 100,
$$

where $\overline{B E_{l}\left(26^{\circ} \mathrm{C}\right)}$ is the average value of the backscattered energy, expressed in an arbitrary units, of each harmonic at the baseline temperature. This value was added to the denominator in order to stabilize the division. $\overline{B E_{1}\left(26^{\circ} \mathrm{C}\right)}, \overline{B E_{2}\left(26^{\circ} \mathrm{C}\right)}$ and $\overline{B E_{3}\left(26^{\circ} \mathrm{C}\right)}$ were $0.052,0.0064$ and $1.82 \times 10^{-5}$ in the absence of vibration in the phantom and the values were $0.0698,0.007,1.7 \times 10^{-5}$ in the presence of vibration, respectively. The percentage changes in the first, second and third 
harmonics were related to temperature by using calibration curves that were derived in our previous work [83].

The conventional echo shift technique was performed by taking cross-correlation between each two frames obtained at each temperature with a window size of $1 \times \tau$ i.e.0.077 $\mu$ s $(118 \mu \mathrm{m})$, and an overlap of 50\%. 2D gradient maps were obtained by differentiating the cumulative echo shifts along the axial direction.

\subsection{Results}

Figure 5-2 and Figure 5-3 show estimated 2D temperature maps using the change in backscattered energies of the acoustic harmonics $\left(C B E_{1}, C B E_{2}\right.$ and $\left.C B E_{3}\right)$ generated in the tissue mimicking gel phantom when the temperature was changed from $26^{\circ} \mathrm{C}$ to $46^{\circ} \mathrm{C}$. Figure $5-4$ shows the gradient maps using the conventional echo-shift technique. The temperatures measured in the center of heated region by the thermocouple were $31,38,42$ and $46^{\circ} \mathrm{C}$ as denoted in the figures. The vibration meter measured maximum displacement of $1.455,0.221$ and $1.030 \mathrm{~mm}$ in $x, y$ and $z$ directions. The frequency of the motion was approximately $0.8 \mathrm{~Hz}$. 


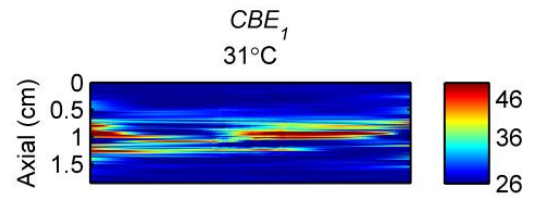

$38^{\circ} \mathrm{C}$

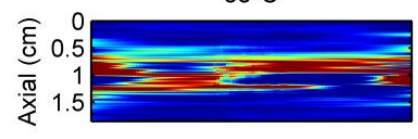

$42^{\circ} \mathrm{C}$

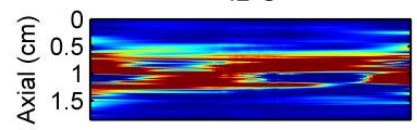

$46^{\circ} \mathrm{C}$

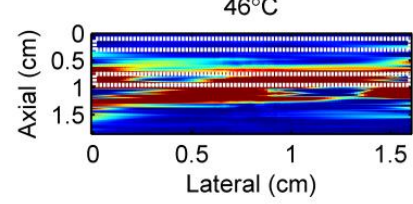

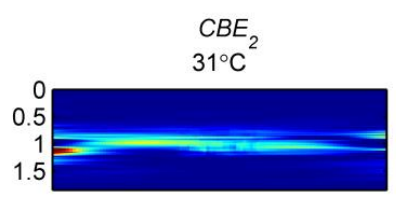

$38^{\circ} \mathrm{C}$
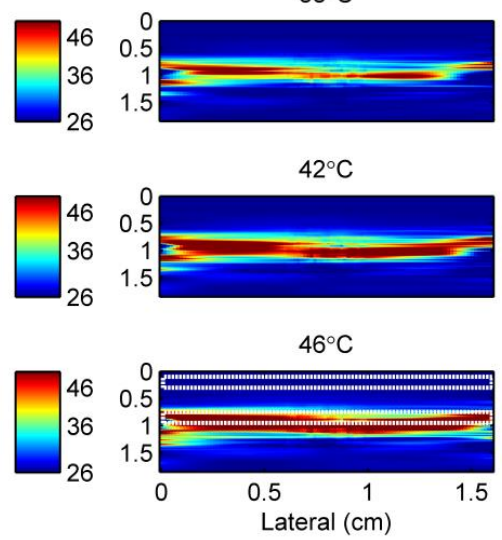

$42^{\circ} \mathrm{C}$

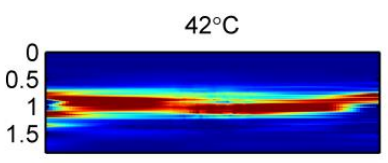

$46^{\circ} \mathrm{C}$

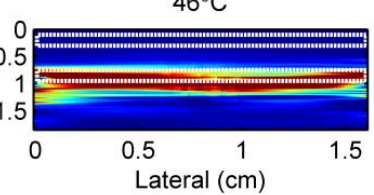

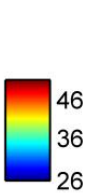

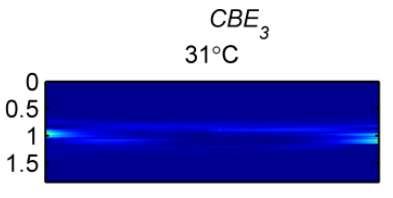

$38^{\circ} \mathrm{C}$

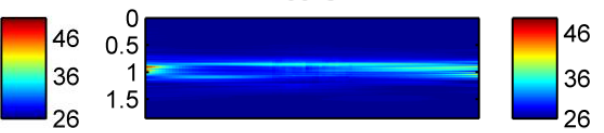

$42^{\circ} \mathrm{C}$

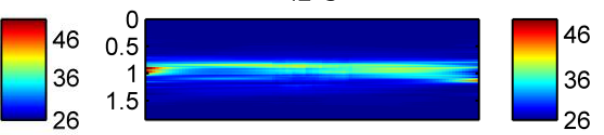

$46^{\circ} \mathrm{C}$

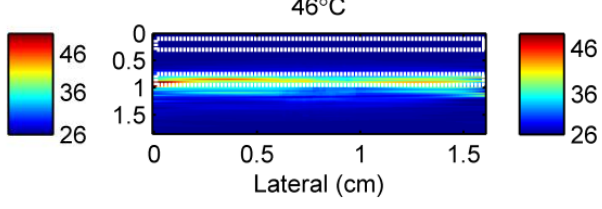

Figure 5-2: Estimated 2D temperature maps in tissue mimicking gel phantom obtained using CBE1 (left), CBE2 (middle), and CBE3 (right) while the temperature was cooling down from $46^{\circ} \mathrm{C}$ to $26^{\circ} \mathrm{C}$ and no vibration was present in the phantom. The temperatures measured by the inserted thermocouple were $31,38,42$ and $46^{\circ} \mathrm{C}$ at the center of heated region. The color bars represent the temperature in ${ }^{\circ} \mathrm{C}$.

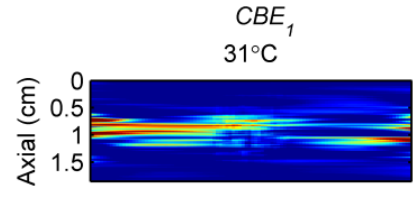

$38^{\circ} \mathrm{C}$

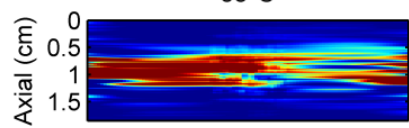

$42^{\circ} \mathrm{C}$

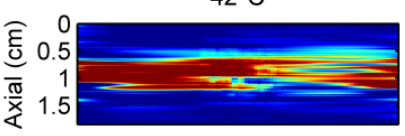

$46^{\circ} \mathrm{C}$

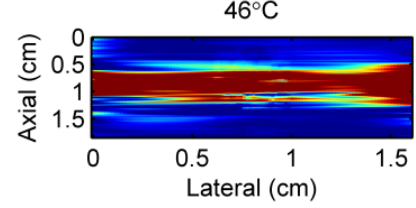

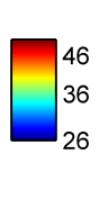

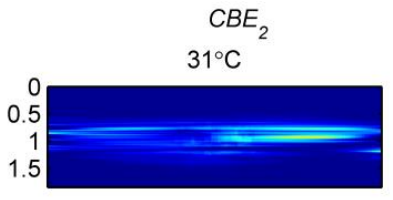

$38^{\circ} \mathrm{C}$
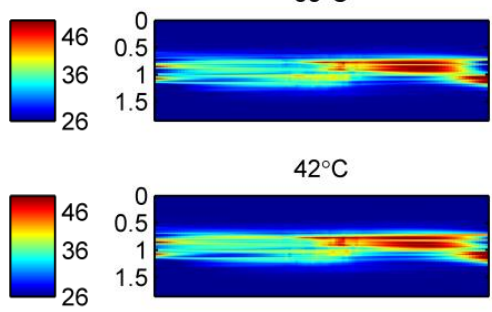

$42^{\circ} \mathrm{C}$

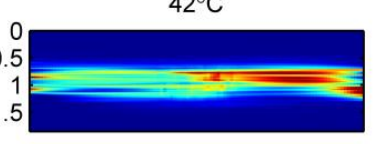

$46^{\circ} \mathrm{C}$

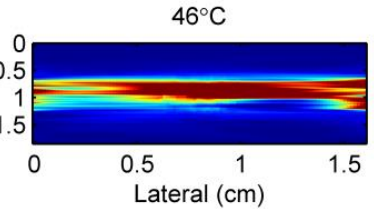

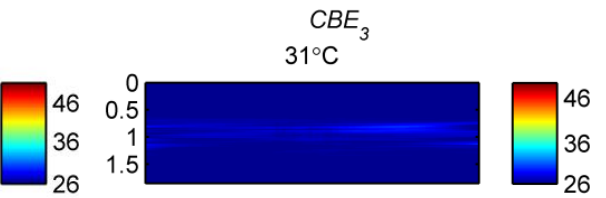

$38^{\circ} \mathrm{C}$

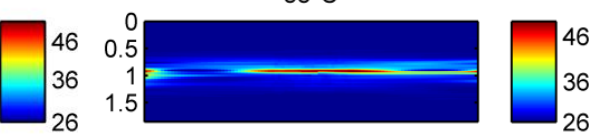

$42^{\circ} \mathrm{C}$

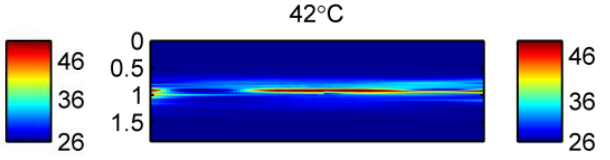

$46^{\circ} \mathrm{C}$
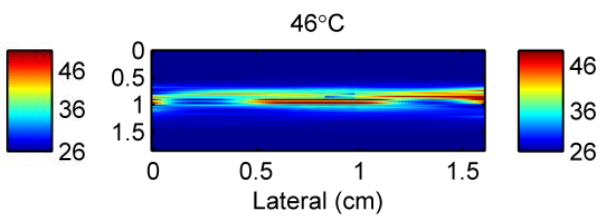

Figure 5-3: Estimated 2D temperature maps in tissue mimicking gel phantom obtained using CBE1 (left), CBE2 (middle), and CBE3 (right) while the temperature was elevated from 26 to $46^{\circ} \mathrm{C}$ with the presence of vibration in the phantom. The temperatures measured by the inserted thermocouple were $31,38,42$ and $46^{\circ} \mathrm{C}$ at the center of heated region. The color bars represent the temperature in ${ }^{\circ} \mathrm{C}$. 

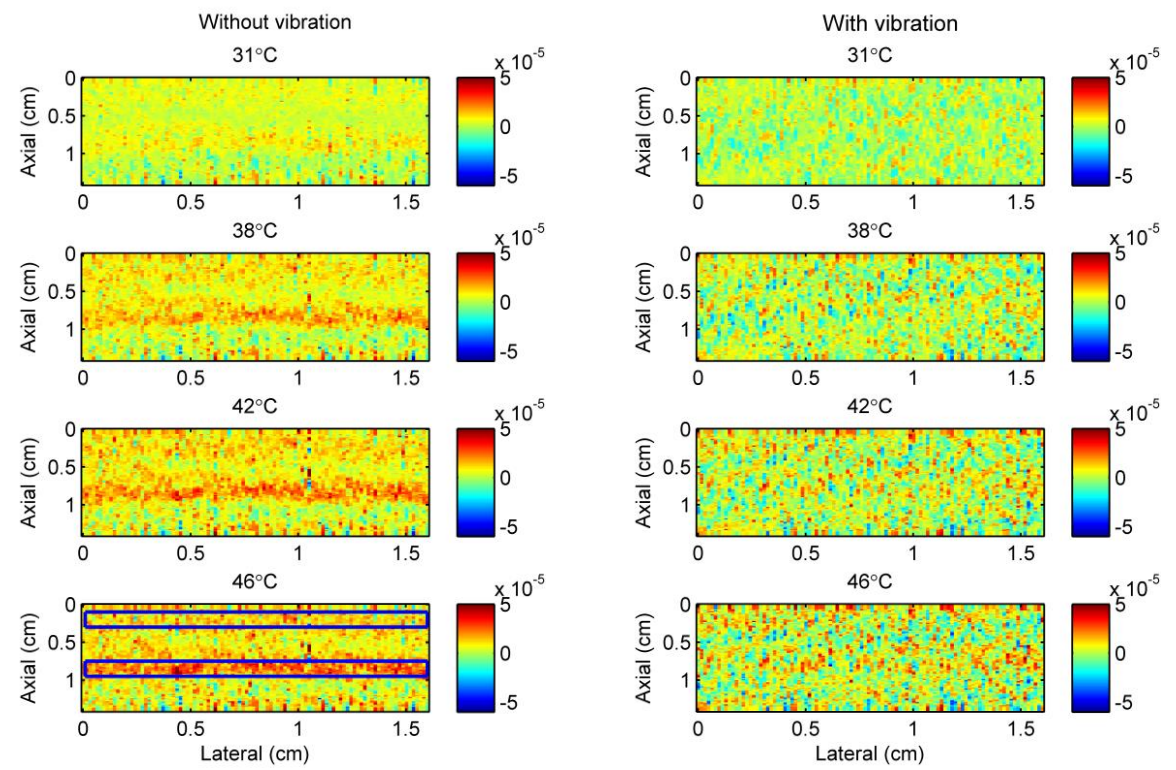

Figure 5-4: 2D gradient maps in tissue mimicking gel phantom obtained using the conventional echo-shift technique without (left) and with (right) presence of vibration in the phantom. The temperatures measured by the inserted thermocouple were $31,38,42$ and $46^{\circ} \mathrm{C}$ at the center of heated region. The color bars represent the axial gradient of the cumulative time shifts in units of $\mathrm{s} / \mathrm{m}$.

\subsection{Discussion}

Figure 5-2 and Figure 5-3 show that the acoustic harmonics generated by nonlinear ultrasound wave propagation can be used to estimate temperature in a sample where only a localized volume is heated. The results also show that this technique is nearly unaffected by the vibration induced in the phantom. Temperature maps show that the temperature was overestimated, especially for temperatures derived using the fundamental frequency $\left(C B E_{1}\right)$. There were regions that the temperature measured by the thermocouple was 31,38 or $42^{\circ} \mathrm{C}$ but the maps estimate the temperature to be $46^{\circ} \mathrm{C}$. It should be noted that the thermocouple samples the temperature at one location, $2 \mathrm{~mm}$ away from the heating source (Figure 5-1), which may partially contribute to the discrepancy. Temperature accuracy improved by using higher harmonic number $\left(C B E_{2}\right.$ and $\left.C B E_{3}\right)$. 
Figure 5-4 demonstrates that the gradient map obtained using the conventional echo-shift technique shows the heated region in the phantom only in the case where the measurement was not affected by vibrations. However, it is important to note that there has been no motion compensation technique applied for obtaining these maps. It is anticipated that applying a motion compensation technique along with a very high-frame-rate data acquisition $[39,106]$ will reduce the effect of motion artifacts in both methods. In this study, we were not able to obtain temperature maps using the echo-shift technique because of not having the medium dependent parameter $k$ as defined in Eq. 5- 1 .

Contrast to Noise Ratio (CNR) and Signal to Noise Ratio (SNR) were calculated as metrics in order to compare the image qualities of temperature maps between the two techniques and for the results of each technique in the presence and absence of vibration (Table 5-1 and Table 5-2). CNR and SNR were calculated using [54]:

$$
\begin{gathered}
C N R=\frac{\left|\mu_{\text {heated }}-\mu_{\text {unheated }}\right|}{\sqrt{\sigma_{\text {heated }}^{2}+\sigma_{\text {unheated }}^{2}}}, \\
S N R=\frac{\mu_{\text {heated }}}{\sigma_{\text {heated }}}
\end{gathered}
$$

where $\mu_{\text {heated }}$ and $\mu_{\text {unheated }}$ are the mean pixel values inside and outside the heated regions, respectively, and $\sigma_{\text {heated }}$ and $\sigma_{\text {unheated }}$ are the standard deviation of pixel values inside and outside the heated regions, respectively. The regions of interest for calculating the mean and the standard deviation inside and outside the heated areas were selected as horizontal rectangles as shown in Figure 5-2 and Figure 5-4. 


\begin{tabular}{ccccc}
\hline & $\begin{array}{c}C B E_{1} \\
\text { (no-vib.) }\end{array}$ & $\begin{array}{c}\mathrm{CBE}_{2} \\
\text { (no-vib.) }\end{array}$ & $\begin{array}{c}\mathrm{CBE}_{3} \\
\text { (no-vib.) }\end{array}$ & $\begin{array}{c}\text { Echo-shift } \\
\text { (no-vib.) }\end{array}$ \\
\hline SNR & $1.91 \pm 0.24$ & $2.1 \pm 0.62$ & $2.68 \pm 1.04$ & $1.71 \pm 0.54$ \\
CNR & $1.78 \pm 0.24$ & $2.08 \pm 0.62$ & $2.6 \pm 0.99$ & $0.75 \pm 0.12$ \\
\hline
\end{tabular}

Table 5-1: The mean and the standard deviation of calculated SNR and CNR values for temperature and gradient maps without the presence of vibration in the phantom.

\begin{tabular}{ccccc}
\hline & $\begin{array}{c}C B E_{1} \\
\text { (vib.) }\end{array}$ & $\begin{array}{c}C B E_{2} \\
\text { (vib.) }\end{array}$ & $\begin{array}{c}C B E_{3} \\
\text { (vib.) }\end{array}$ & $\begin{array}{c}\text { Echo-shift } \\
\text { (vib.) }\end{array}$ \\
\hline SNR & $1.55 \pm 0.55$ & $2.09 \pm 0.67$ & $2 \pm 0.85$ & $0.34 \pm 0.3$ \\
CNR & $1.48 \pm 0.54$ & $2.07 \pm 0.67$ & $1.98 \pm 0.81$ & $0.09 \pm 0.02$ \\
\hline
\end{tabular}

Table 5-2: The mean and the standard deviation of calculated SNR and CNR values for temperature and gradient maps with the presence of vibration in the phantom.

The results show that the CNR is higher in the images obtained using the nonlinear ultrasound technique compared to the echo-shift technique. The SNR and CNR values were significantly reduced in the image obtained using the echo-shift technique in the presence of vibration in the sample compared to the one without vibration. The mean SNR and CNR reduced from 1.71 and 0.75 to 0.34 (by $1 / 5$ times) and 0.09 (by 1/8.3 times) in the presence of phantom vibration. However, the SNR and CNR values in images obtained using the $C B E_{1}, C B E_{2}$, and $C B E_{3}$ of the harmonics reduced by approximately $0.81,0.9$ and 0.74 times in the phantom with vibration compared to the one without.

The magnitude of human organ motion depends on anatomical site. It has to be noted that the degree of motion induced in the phantom in this study is smaller than the typical motion of internal organs such as liver, kidney and pancreas under normal breathing condition [107]. 
However, the magnitude of the motion is comparable to that of such organs in forced breath holding condition and the frequency of the motion is similar to the heart rate [107].

\subsection{Conclusion}

The results obtained in this study indicate that noninvasive temperature estimation using the backscattered energies of the harmonics is feasible in a tissue-mimicking sample where only localized volumes were heated. In the absence of vibration in the sample, 2D temperature and gradient maps were obtained using the $C B E$ of harmonics and the RF echo-shift technique. In the presence of the vibration that is mechanically induced in the sample and with no motion compensation technique applied, noninvasive thermometry was feasible only by using the backscattered energies of the harmonics approach. It is anticipated that applying motion compensation will improve the performances of both temperature estimation methods.

\subsection{Acknowledgements}

The authors would like to acknowledge the technical assistance of Arthur Worthington from Dept. of Physics, Ryerson University, Toronto, Canada. This work was partially funded by the Ontario Research Fund- Research Excellence (ORF-RE) grant and the Natural Sciences and Engineering Research Council of Canada (NSERC Discovery grants) that were awarded to J. Tavakkoli and M. C. Kolios. Funding to purchase the equipment was provided by the Canada Foundation for Innovation, the Ontario Ministry of Research and Innovation, the Canada Research Chairs Program, and the Ryerson University Research Fund. 


\section{Chapter 6: Thesis conclusions and future work}

\subsection{Summary and Conclusions}

The research work in this dissertation describes the temperature dependence of acoustic harmonics generated by nonlinear ultrasound wave propagation in several media, and explores a new noninvasive thermometry approach that analyzes the backscattered energy of the harmonics. This section summarizes the significant findings and conclusions of the research work.

The effect of temperature on harmonic generation in water at various pulse transmit frequencies between 1 to $20 \mathrm{MHz}$ was studied in Chapter 2. Simulations were conducted using a modified KZK nonlinear wave equation that accounts for temperature dependent medium parameters. For measurements, two single-element focused transducers were used to transmit tone bursts at $3.3 \mathrm{MHz}$ and $13 \mathrm{MHz}$. The simulation and measurement results show that the temperature dependence of the harmonic generation is different at various frequencies. The harmonic amplitudes decrease with temperature elevation from 26 to $46^{\circ} \mathrm{C}$ at the transmit frequencies of 1 and 3.3 MHz. On the other hand, the harmonic amplitudes increased with temperature at 13 and $20 \mathrm{MHz}$. This variation in the temperature dependence of the harmonic generation at different frequencies is due to the interplay between the mechanisms of absorption, nonlinearity and focusing gain as fully discussed in Chapter 2.

Chapter 3 shows the temperature dependence of acoustic harmonics generated in a tissuemimicking attenuating liquid at two transmit frequencies of 5 and $13 \mathrm{MHz}$. The temperature dependent KZK model was used to simulate nonlinear wave propagation in the medium at temperatures of $26,31,36,41$ and $46^{\circ} \mathrm{C}$. Measurements were performed using two single- 
element focused transducers at frequencies of $5 \mathrm{MHz}$ and $13 \mathrm{MHz}$ in an attenuating liquid at the same temperatures. The harmonics showed strong temperature dependencies in the liquid at both frequencies (more so at $13 \mathrm{MHz}$ ), which is due to the combined effects of an increase in the nonlinear parameter $(N)$ and the focusing gain $(G)$, and a decrease in the absorption parameter (A).

In Chapter 4, the change in pressure amplitude and backscattered energy of the harmonics as a function of temperature were obtained in tissue-mimicking gel phantoms and ex vivo bovine muscle tissues at $13 \mathrm{MHz}$ in pulse-echo mode. The change in backscattered energy of the harmonics versus temperature were then used to generate temperature maps in chapter 5. In Chapter 5, we proposed a new noninvasive ultrasound-based thermometry technique using the change in the backscattered energy of harmonics in a tissue-mimicking gel phantom where only a localized volume was heated. The motion artifact susceptibility of this technique was compared with the conventional echo-shift technique. Unlike our proposed harmonics-based method, the temperature gradient maps obtained using the echo-shift technique were not capable of showing the heated region in the presence of the vibration induced in the sample. This shows the high sensitivity of the echo-shift thermometry technique to motion comparing to our proposed harmonic-based method.

\subsection{Future Work}

The harmonics generation was studied as a function of temperature and transmit pulse frequency in water and a tissue-mimicking liquid using simulations and experiments to understand the mechanism of action of harmonic generation with temperature change at different frequencies. The temperature dependence of harmonics was experimentally obtained in ex vivo tissue only at $13 \mathrm{MHz}$. The next step is to study the temperature dependence of harmonics in 
tissue at various frequencies ( 1 to $13 \mathrm{MHz}$ ) and source pressures in order to find the optimal source parameters for nonlinear tissue thermometry. The temperature dependence of harmonics could also be obtained in more heterogeneous tissues containing fatty tissues.

As shown in chapter 4, the standard deviations of the harmonics versus temperature were high. Calibration curves could be improved by: 1) reducing the heating time to avoid tissue decomposition and 2) increasing the SNR of the recorded harmonics by sending higher pressure signals or using broader band transducers.

2D temperature maps should be obtained in ex vivo tissue samples using both acoustic harmonics and echo-shift techniques, and the accuracy of both techniques should be compered. The sensitivity of both techniques to motion artifacts could also be studied when motion compensation algorithms are applied.

Temperature can be locally increased in the tissue using a focused ultrasound transducer, which is a more practical way of heating rather than circulating hot water in a needle. Thermometry may also be possible using the same transducer. The thermometry technique using the acoustic harmonics could be implemented in in vivo studies after obtaining the temperature dependence of the harmonics in the tissue of interest.

The possibility of using harmonics in detecting thermal lesions could also be the topic of a future study. The attenuation coefficient increases when tissue undergoes coagulation and the increase in attenuation causes reduction in harmonic generation. The change in the trend of harmonic generation from increasing in pre-coagulation to decreasing in post-coagulation could be used to detect tissue ablation.

There are several limitations that apply to the harmonics-based method. The results show that the harmonics are more sensitive to temperature at higher transmit frequencies. However, 
there is a tradeoff between the frequency and the penetration depth of the ultrasound beam. Imaging with higher frequencies reduces the imaging depth. The temperature range covered in this work was from 26 to $46^{\circ} \mathrm{C}\left(\Delta T=20^{\circ} \mathrm{C}\right)$. However, the normal physiological temperature is $37^{\circ} \mathrm{C}$ which is increased to around $44^{\circ} \mathrm{C}$ in hyperthermia $\left(\Delta T \approx 7^{\circ} \mathrm{C}\right)$. The temperature sensitivity of the harmonics reported in this work was for a temperature change of $20^{\circ} \mathrm{C}$ but the practical temperature change in clinic is about $7^{\circ} \mathrm{C}$. Therefore, the harmonics will have lower sensitivity to temperature within a smaller temperature range and the heated regions in temperature maps will have a lower contrast to the unheated regions which will have a baseline temperature of $37^{\circ} \mathrm{C}$ rather than $26^{\circ} \mathrm{C}$. It was shown that the temperature dependence of harmonic generation is dependent on the source frequency. On the other hand, harmonic generation also depends on the source pressure amplitude and the distance that the ultrasound beam propagates. Varying the source pressure amplitude and the focal length could therefore lead to changes in the reported results. An extension of this work will be to investigate the effect of source pressure amplitude and the beam propagation distance on harmonics generation with varying temperature. 


\section{Bibliography}

[1] J. H. Howard, C.-W. Tzeng, J. K. Smith, D. E. Eckhoff, J. S. Bynon, T. Wang, J. P. Arnoletti and M. J. Heslin, "Radiofrequency Ablation for Unresectable Tumors of the Liver," The American Surgeon, vol. 74, no. 7, pp. 594-600, 2008.

[2] M. K. Kang, M. S. Kim and J. H. Kim, "Clinical outcomes of mild hyperthermia for locally advanced rectal cancer treated with preoperative radiochemotherapy," International Journal of Hyperthermia, vol. 27, no. 5, pp. 482-490, 2011.

[3] S. B. Field and J. W. Hand, An Introduction to the Practical Aspects of Clinical Hyperthermia, Philadelphia: Taylor \& Francis, 1990.

[4] J. van der Zee, "Heating the patient: a promising approach?," Annals of Oncology, vol. 13, no. 8, pp. 1173-1184, 2002.

[5] J. P. May and S.-D. Li, "Hyperthermia-induced drug targeting," Expert Opinion on Drug Delivery, vol. 10, no. 4, pp. 511-527, 2013.

[6] G. A. Koning, A. M. M. Eggermont, L. H. Lindner and T. L. M. ten Hagen, "Hyperthermia and Thermosensitive Liposomes for Improved Delivery of Chemotherapeutic Drugs to Solid Tumors," Pharmaceutical Research, vol. 27, no. 8, pp. 1750-1754, 2010.

[7] H. Grüll and S. Langereis, "Hyperthermia-triggered drug delivery from temperaturesensitive liposomes using MRI-guided high intensity focused ultrasound," Drug Delivery Research in Europe, vol. 161, no. 2, pp. 317-327, 2012.

[8] S. A. Sapareto and W. C. Dewey, "Thermal dose determination in cancer therapy," International Journal of Radiation Oncology Biology Physics, vol. 10, no. 6, pp. 787-800, 1984.

[9] M. A. O’Reilly and K. Hynynen, "Emerging non-cancer applications of therapeutic ultrasound," International Journal of Hyperthermia, vol. 31, no. 3, pp. 1-9, 2015.

[10] A. Klein, W. Bäumler, M. Landthaler and P. Babilas, "Laser thermal therapy of benign skin tumours: Review and update," International Journal of Hyperthermia, vol. 27, no. 8, pp. 762-770, 2011. 
[11] A. C. Steger, W. R. Lees, K. Walmsley and S. G. Bown, "Interstitial Laser Hyperthermia: A New Approach To Local Destruction Of Tumours," British Medical Journal, vol. 299, no. 6695 , pp. $362-365,1989$.

[12] M. G. Skinner, M. N. Iizuka , M. C. Kolios and M. D. Sherar, "A theoretical comparison of energy sources-microwave, ultrasound and laser-for interstitial thermal therapy," Physics in Medicine and Biology, vol. 43, no. 12, p. 3535-3547, 1998.

[13] H. J. Schwarzmaier, F. Eickmeyer, F. Ulrich and V. U. Fiedler, "Basic Principles of Laser Induced Interstitial Thermotherapy in Brain Tumors," Medical Laser Application, vol. 17, no. 2, pp. 147-158, 2002.

[14] X. Huang, P. K. Jain, I. H. El-Sayed and M. A. El-Sayed, "Plasmonic photothermal therapy (PPTT) using gold nanoparticles," Lasers in Medical Science, vol. 23, no. 3, pp. 217-228, 2008.

[15] X. Huang, I. H. El-Sayed, W. Qian and M. A. El-Sayed, "Cancer cell imaging and photothermal therapy in the near-infrared region by using gold nanorods," Journal of the American Chemical Society, vol. 128, pp. 2115-2120, 2006.

[16] L. A. Dombrovsky, V. Timchenko, M. Jackson and G. H. Yeoh, "A combined transient thermal model for laser hyperthermia of tumors with embedded gold nanoshells," International Journal of Heat and Mass Transfer, vol. 54, no. 25, pp. 5459-5469, 2011.

[17] T. B. Huff, L. Tong, M. N. Hansen, J.-X. Cheng, A. Wei and A. Wei, "Hyperthermic effects of gold nanorods on tumor cells," Nanomedicine, vol. 2, no. 1, pp. 125-132, 2007.

[18] G. S. Terentyuk, G. N. Maslyakova, N. G. Khlebtsov, B. N. Khlebtsov, G. G. Akchurin, I. L. Maksimova and V. V. Tuchin, "Laser-induced tissue hyperthermia mediated by gold nanoparticles: toward cancer phototherapy," Journal of Biomedical Optics, vol. 14, no. 2, 2009.

[19] W. Cai, T. Gao, H. Hong and J. Sun, "Applications of gold nanoparticles in cancer nanotechnology," Nanotechnology, Science and Applications, vol. 2008, pp. 17-32, 2008.

[20] T. P. Ryan, P. F. Turner and B. Hamilton, "Interstitial microwave transition from hyperthermia to ablation: Historical perspectives and current trends in thermal therapy," International Journal of Hyperthermia, vol. 26, no. 5, pp. 415-433, 2010. 
[21] P. R. Stauffer, P. Maccarini, K. Arunachalam, O. Craciunescu, C. Diederich, T. Juang, F. Rossetto, J. Schlorff, A. Milligan, J. Hsu, P. Sneed and Z. Vujaskovic, "Conformal microwave array (CMA) applicators hyperthermia diffuse chest wall recurrence," International Journal of Hyperthermia, vol. 26, no. 7, pp. 686-698, 2010.

[22] R. W. Y. Habash, R. Bansal, D. Krewski and H. T. Alhafid, "Thermal therapy, part 2: hyperthermia techniques," Critical Reviews in Biomedical Engineering, vol. 34, no. 6, pp. 491-542, 2006.

[23] M. M. Sackenheim, "Radio Frequency Ablation The Key to Cancer Treatment," Journal of Diagnostic Medical Sonography, vol. 19, no. 2, pp. 88-92, 2003.

[24] H. Rhim, S. N. Goldberg, G. D. Dodd, L. Solbiati, H. K. Lim, M. Tonolini and O. K. Cho, "Essential Techniques for Successful Radio-frequency Thermal Ablation of Malignant Hepatic Tumors," RadioGraphics, vol. 21, pp. S17-S35, 2001.

[25] C. L. Brace, "Radiofrequency and microwave ablation of the liver, lung, kidney, and bone: what are the differences?," Current Problems in Diagnostic Radiology, vol. 38, no. 3, pp. 135-143, 2009.

[26] A. Cheung and A. Neyzari, "Deep local hyperthermia for cancer therapy: external electromagnetic and ultrasound techniques," Cancer Resarch, vol. 44, pp. 4736s-4744s, 1984.

[27] Y. Laufer and G. Dar, "Effectiveness of thermal and athermal short-wave diathermy for the management of knee osteoarthritis: A systematic review and meta-analysis," Osteoarthritis and Cartilage, vol. 20, no. 9, pp. 957-966, 2012.

[28] N. Shields, J. Gormley and N. O'Hare, "Short-wave diathermy: a review of existing clinical trials," Physical Therapy Reviews, vol. 6, pp. 101-118, 2001.

[29] T. A. Leslie and J. E. Kennedy, "High-intensity Focused Ultrasound Principles, Current Uses, and Potential for the Future," Ultrasound Quarterly, vol. 22, no. 4, pp. 263-272, 2006.

[30] J. Tavakkoli and N. T. Sanghvi, "Ultrasound-guided HIFU and Thermal Ablation," in Therapeutic Ultrasound: Mechanisms to Applications, Hauppauge, Nova Science Publishers, 2011, pp. 137-161. 
[31] G. R. ter Haar, "Therapeutic applications of ultrasound," Progress in Biophysics \& Molecular Biology, vol. 93, pp. 111-129, 2007.

[32] C. J. Diederich and K. Hynynen, "Ultrasound technology for hyperthermia," Ultrasound in Medicine and Biology, vol. 25, no. 6, p. 871-887, 1999.

[33] E. G. Moros, W. L. Straube, R. J. Myerson and X. Fan, "The impact of ultrasonic parameters on chest wall hyperthermia," International Journal of Hyperthermia, vol. 16, no. 6 , pp. 523-538, 2000.

[34] G. t. Haar and C. Coussios, "High intensity focused ultrasound: Physical principles and devices," International Journal of Hyperthermia, vol. 23, no. 2, pp. 89-104, 2007.

[35] R. M. Arthur, D. Basu, Y. Guo, J. Trobaugh and E. Moros, "3-D in vitro estimation of temperature using the change in backscattered ultrasonic energy," IEEE Transactions on Ultrasonics Ferroelectrics and Frequency Control, vol. 57, no. 8, pp. 1724-1733, 2010.

[36] I. Rivens, A. Shaw, J. Civale and H. Morris, "Treatment monitoring and thermometry for therapeutic focused ultrasound," International Journal of Hyperthermia, vol. 23, no. 2, pp. 121-139, 2007.

[37] S. Dasgupta, R. K. Banerjee and M. R. Myers, "Beam localization in HIFU temperature measurements using thermocouples, with application to cooling by large blood vessels," Ultrasonics, vol. 51, no. 2, pp. 171-180, 2011.

[38] M. A. Lewis, R. M. Staruch and R. Chopra, "Thermometry and ablation monitoring with ultrasound," International Journal of Hyperthermia, vol. 31, pp. 163-181, 2015.

[39] D. Liu and E. S. Ebbini, "Real-time Two-dimensional temperature estimation using diagnostic ultrasound," IEEE Transactions on Biomedical Engineering, vol. 57, pp. 12-16, 2010.

[40] V. Rieke and K. B. Pauly, "MR thermometry," Journal of Magnetic Resonance Imaging , vol. 27, no. 2, pp. 376-390, 2008.

[41] R. M. Arthur, W. L. Straube, J. W. Trobaugh and E. G. Moros, "Non-invasive estimation of hyperthermia temperatures with ultrasound," International Journal of Hyperthermia, vol. 21, no. 6, pp. 589-600, 2005.

[42] I. V. Larina, K. V. Larin and R. O. Esenaliev, "Real-time optoacoustic monitoring of 
temperature in tissues," Journal of Physics D-applied Physics, vol. 38, no. 15, pp. 26332639, 2005.

[43] G. K. Hesley, K. R. Gorny and D. A. Woodrum, "MR-guided focused ultrasound for the treatment of uterine fibroids," CardioVascular and Interventional Radiology, vol. 36, no. 1, pp. 5-13, 2013.

[44] Y. S. Kim, B. Keserci, A. Partanen, H. Rhim, H. K. Lim, M. J. Park and M. O. Köhler, "Volumetric MR-HIFU ablation of uterine fibroids: Role of treatment cell size in the improvement of energy efficiency," European Journal of Radiology, vol. 81, pp. 36523659, 2012.

[45] L. V. Wang and S. Hu, "Photoacoustic tomography: in vivo imaging from organelles to organs," Science, vol. 335, no. 6075, p. 1458-1462, 2012.

[46] E. Petrova, S. Ermilov, R. Su, V. Nadvoretskiy, A. Conjusteau and A. Oraevsky, "Using optoacoustic imaging for measuring the temperature dependence of Grüneisen parameter in optically absorbing solutions," Optics Express, vol. 21, no. 21, pp. 25077-25090, 2013.

[47] J. Yao, K. Haixin, S. Tai, Y. Zhou and L. V. Wang, "Absolute photoacoustic thermometry in deep tissue," Optics Letters, vol. 38, no. 24, pp. 5228-5231, 2013.

[48] C. Li and L. V. Wang, "Photoacoustic tomography and sensing in biomedicine," Physics in Medicine and Biology, vol. 54, no. 19, p. R59-R97, 2009.

[49] M. Pramanik, T. N. Erpelding, L. Jankovic and L. V. Wang, "Tissue temperature monitoring using thermoacoustic and photoacoustic techniques," Photons Plus Ultrasound: Imaging and Sensing, vol. 14, no. 5, pp. 1-10, 2010.

[50] M. J. Choi, S. R. Guntur and J. M. Lee, "Changes in ultrasonic properties of liver tissue in vitro during heating-cooling cycle concomitant with thermal coagulation," Ultrasound in Medicine and Biology, vol. 37, pp. 2000-2012, 2011.

[51] E. J. Jackson, C. C. Coussios and R. O. Cleveland, "Nonlinear acoustic properties of ex vivo bovine liver and the effects of temperature and denaturation," Physics in Medicine and Biology, vol. 59, pp. 3223-3238, 2014.

[52] K. W. A. van Dongen and M. D. Verwei, "A feasibility study for non-invasive thermometry using non-linear ultrasound," International Journal of Hyperthermia, vol. 27, 
no. 6, pp. 612-624, 2011.

[53] X. Liu, X. Gong, C. Yin, J. Li and D. Zhang, "Noninvasive Estimation of Temperature Elevations in Biological Tissues Using Acoustic Nonlinearity Parameter Imaging," Ultrasound in Medicine and Biology, vol. 34, no. 3, pp. 414-424, 2008.

[54] R. S. C. Cobbold, Foundation of Biomedical Ultrasound, Oxford University Press, 2007.

[55] C. R. Hill, J. C. Bamber and G. R. ter Haar, Physical Principles of Medical Ultrasound, Second Edition, Chichester: John Wiley \& Sons, Ltd, 2005.

[56] U. Techavipoo, T. Varghese, Q. Chen, T. A. Stiles , J. A. Zagzebski and G. R. Frank, "Temperature dependence of ultrasonic propagation speed and attenuation in excised canine liver tissue measured using transmitted and reflected pulses," Journal of The Acoustical Society of America, vol. 115, no. 6, pp. 2859-2865, 2004.

[57] C. A. Damianou , N. T. Sanghvi , F. J. Fry and R. Maassmoreno, "Dependence of ultrasonic attenuation and absorption in dog soft tissues on temperature and thermal dose," Journal of The Acoustical Society of America, vol. 102, pp. 628-634, 1997.

[58] J. C. Bamber and C. R. Hill, "Ultrasonic attenuation and propagation speed in mammalian tissues as a function of temperature," Ultrasound in Medicine and Biology, vol. 5, no. 2, pp. 149-157, 1979.

[59] N. Parmar and M. C. Kolios, "An investigation of the use of transmission ultrasound to measure acoustic attenuation changes in thermal therapy," Medical \& Biological Engineering \& Computing, vol. 44, no. 7, pp. 583-591, 2006.

[60] G. Ghoshal, A. C. Luchies, J. P. Blue and M. L. Oelze, "Temperature dependent ultrasonic characterization of biological media," The Journal of the Acoustical Society of America, vol. 130, no. 4, p. 2203-2211, 2011.

[61] P. M. Gammell, D. H. Le Croissette and R. C. Heyser, "Temperature and frequency dependence of ultrasonic attenuation in selected tissues," Ultrasound in Medicine \& Biology, vol. 5, no. 3, pp. 269-277, 1979.

[62] R. M. Arthur,, W. L. Straube , J. D. Starman and E. G. Moros, "Noninvasive temperature estimation based on the energy of backscattered ultrasound," Medical Physics, vol. 30, no. 6, pp. 1021-1109, 2003. 
[63] R. M. Arthur, W. L. Straube and G. Moros, "In vivo change in ultrasonic backscattered energy with temperature in motion-compensated images," International Journal of Hyperthermia, vol. 24, no. 5, pp. 389-398, 2008.

[64] W. L. Straube and R. M. Arthur, "Theoretical estimation of the temperature dependence of backscattered ultrasonic power for noninvasive thermometry," Ultrasound in Medicine and Biology, vol. 20, no. 9, pp. 915-922, 1994.

[65] R. M. Vlad, G. J. Czarnota, A. Giles , M. D. Sherar , J. W. Hunt and M. C. Kolios, "Highfrequency ultrasound for monitoring changes in liver tissue during preservation," Physics in Medicine and Biology, vol. 50, no. 2, pp. 197-213, 2005.

[66] X. Li, . G. Ghoshal, R. J. Lavarello and M. L. Oelze, "Exploring potential mechanisms responsible for observed changes of ultrasonic backscattered energy with temperature variations," Medical Physics, vol. 41, no. 5, pp. 1-9, 2014.

[67] C. Simon, P. VanBaren and E. S. Ebbini, "Two-dimensional temperature estimation using diagnostic ultrasound," IEEE Transactions on Ultrasonics Ferroelectrics and Frequency Control, vol. 45, no. 4, pp. 1088-1099, 1998.

[68] N. R. Miller, J. C. Bamber and P. M. Meaney, "Fundamental limitations of noninvasive temperature imaging by means of ultrasound echo strain estimation," Ultrasound in Medicine and Biology, vol. 28, no. 10, pp. 1319-1333, 2002.

[69] C. H. Seo, Y. Shi, S. W. Huang, K. Kim and M. O'Donnell, "Thermal strain imaging: a review," Interface Focus, vol. 1, pp. 649-664, 2011.

[70] M. Pernot, M. Tanter, J. Bercoff, K. R. Waters and M. Fink, "Temperature Estimation Using Ultrasonic Spatial Compound Imaging," IEEE transactions on ultrasonics, ferroelectrics, and frequency control, vol. 51, no. 5, pp. 606-615, 2004.

[71] M. F. Hamilton and D. T. Blackstock, Nonlinear acoustics, San Diego: Academic Press, 1997.

[72] F. Tranquart, N. Grenier, V. Eder and L. Pourcelot, "Clinical use of ultrasound tissue harmonic imaging," Ultrasound in Medicine and Biology, vol. 25, no. 6, pp. 889-894, 1999.

[73] F. A. Duck, "Nonlinear acoustics in diagnostic ultrasound," Ultrasound in Medicine and 
Biology, vol. 28, no. 1, pp. 1-18, 2002.

[74] M. H. Hasani, S. Gharibzadeh, Y. Farjami and J. Tavakkoli, "Unmitigated numerical solution to the diffraction term in the parabolic nonlinear ultrasound wave equation," The Journal of the Acoustical Society of America, vol. 134, no. 3, pp. 1775-1790, 2013.

[75] M. A. Averkiou and M. F. Hamilton, "Nonlinear distortion of short pulses radiated by plane and focused circular pistons," The Journal of the Acoustical Society of America, vol. 102, no. 5, pp. 2539-2548, 1997.

[76] P. T. Christopher and K. J. Parker, "New approaches to nonlinear diffractive field propagation," The Journal of the Acoustical Society of America, vol. 90, pp. 488-499, 1991.

[77] J. Tavakkoli, D. Cathignol, R. Souchon and O. A. Sapozhnikov, "Modeling of pulsed finite-amplitude focused sound beams in time domain," The Journal of the Acoustical Society of America, vol. 104, pp. 2061-2072, 1998.

[78] R. J. Zemp, J. Tavakkoli and R. S. C. Cobbold, "Modeling of nonlinear ultrasound propagation in tissue from array transducers," The Journal of the Acoustical Society of America, vol. 113, pp. 139-152, 2003.

[79] T. L. Szabo, Diagnostic Ultrasound Imaging: Inside Out, Oxford: Academic Press, 2014.

[80] A. Bouakaz and N. de Jong, "Native tissue imaging at superharmonic frequencies," IEEE Transactions on Ultrasonics, Ferroelectrics and Frequency Control, vol. 50, pp. 496-506, 2003.

[81] D. Zhang, X. F. Gong and X. Chen, "Experimental imaging of the acoustic nonlinearity parameter B/A for biological tissues via a parametric array," Ultrasound in Medicine and Biology, vol. 27, pp. 1359-1365, 2001.

[82] E. A. Both, "Feasibility study of temperature estimation based on nonlinear acoustics," M.Sc. dissertation, Delft University of Technology, 2010.

[83] B. Maraghechi, M. C. Kolios and J. Tavakkoli, "Temperature dependence of nonlinear acoustic harmonics in ex vivo tissue and tissue-mimicking phantom," International Journal of Hyperthermia, vol. 31, pp. 666-673, 2015.

[84] Y. S. Lee and M. F. Hamilton, "Time-domain modeling of pulsed finite amplitude sound 
beams," The Journal of the Acoustical Society of America, vol. 97, pp. 906-917, 1995.

[85] R. T. Beyer, "Parameter of nonlinearity in fluids," The Journal of the Acoustical Society of America, vol. 32, pp. 719-721, 1960.

[86] B. Zeqiri and A. D. Bond, "The influence of wave-form distortion on hydrophone spatialaveraging corrections-Theory and measurement," The Journal of the Acoustical Society of America, vol. 92, pp. 1809-1809, 1992.

[87] M. S. Canney, M. R. Bailey, L. A. Crum, V. A. Khokhlova and O. A. Sapozhnikov, "Acoustic characterization of high intensity focused ultrasound fields: A combined measurement and modeling approach," The Journal of the Acoustical Society of America, vol. 124, pp. 2406-2420, 2008.

[88] W. Keck and R. T. Beyer, "Frequency spectrum of finite amplitude ultrasonic waves in liquids," Physics of Fluids, vol. 3, pp. 346-352, 1960.

[89] C. M. Sehgal, R. C. Bahn and J. F. Greenleaf, "Measurement of the acoustic nonlinearity 10 parameter B/A in human tissues by a thermodynamic method," The Journal of the Acoustical Society of America, vol. 76, pp. 1023-1029, 1984.

[90] C. M. Sehgal, G. M. Brown, R. C. Bahn and J. F. Greenleaf, "Measurement and use of 7 acoustic nonlinearity and sound speed to estimate composition of excised livers," Ultrasound in Medicine \& Biology, vol. 12, pp. 865-874, 1986.

[91] G. S. Darbari, R. P. Singh and G. S. Verma, "Temperature dependence of ultrasonic absorption in mixtures of glycerol and water," II Nuovo Cim, vol. 41, p. 15-24, 1966.

[92] H. Khelladi, F. Plantier, J. L. Daridon and H. Djelouah, "Measurement under high pressure of the nonlinearity parameter B/A in glycerol at various temperatures," Ultrasonics, vol. 49, pp. 668-675, 2009.

[93] M. J. Ernsting, A. Worthington, J. P. Map, T. Tagami, M. C. Kolios and S. D. Li, "Ultrasound drug targeting to tumors with thermosensitive liposomes," Proc IEEE International Ultrasonics Symposium, pp. 1-4, 2011.

[94] J. E. Kennedy, "High intensity focused ultrasound in the treatment of solid tumours," Nature Reviews Cancer, vol. 5, pp. 321-327, 2005.

[95] D. Liu, J. Jiang, J. Bischof, J. Ballard and E. S. Ebbini, "Real-time monitoring of thermal 
and mechanical response to sub-therapeutic HIFU beams in vivo," Ultrasonics, IEEE Symposium, pp. 2254-2257, 2010.

[96] V. Ingle and J. Proakis, Digital Signal Processing Using MATLAB, Stamford: Cengage Learning, 2011.

[97] N. G. Parker and M. J. W. Povey, "Ultrasonic study of the gelation of gelatin: Phase diagram, hysteresis and kinetics," Food Hydrocolloids, vol. 26, pp. 99-107, 2012.

[98] J. R. Cook, R. R. Bouchard and S. Y. Emelianov, "Tissue-mimicking phanphantoms for photoacoustic and ultrasonic imaging," Biomedical Optics Express, vol. 2, p. 3193-3206, 2011.

[99] C. Lafon, V. Zderic, M. L. Noble, J. C. Yuen, P. J. Kaczkowski, O. A. Sapozhnikov, F. Chavrier, L. A. Crum and S. Vaezy, "Gel phantom for use in high-intensity focused ultrasound dosimetry," Ultrasound in Medicine \& Biology, vol. 31, pp. 1383-1389, 2005.

[100] E. L. Madsen, J. A. Zagzebski, R. A. Banjavie and R. E. Jutila, "Tissue mimicking materials for ultrasound phantoms," Med Phys, vol. 5, pp. 391-394, 1978 .

[101] U. Techavipoo, T. Varghese, J. A. Zagzebski, T. Stiles and G. Frank, "Temperature dependence of ultrasonic propagation speed and attenuation in canine tissue," Ultrasonic Imaging, vol. 4, pp. 246-260, 2002.

[102] H. L. Liu, W. L. Lin and Y. Y. Chen, "A fast and conformal heating scheme for producing large thermal lesions using a 2D ultrasound phased array," International Journal of Hyperthermia, vol. 23, pp. 69-82, 2007.

[103] W. C. Dewey, "Arrhenius relationships from the molecule and cell to the clinic," International Journal of Hyperthermia, vol. 25, pp. 3-20, 2009.

[104] M. Bayat, J. R. Ballard and E. S. Ebbini, "In vivo ultrasound thermography in presence of temperature heterogeneity and natural motions," IEEE Transactions on Biomedical Engineering, vol. 62, pp. 450-457, 2015.

[105] B. Maraghechi, M. H. Hasani, M. C. Kolios and J. Tavakkoli, "Temperature dependence of acoustic harmonics generated by nonlinear ultrasound wave propagation in water at various frequencies," The Journal of the Acoustical Society of America, vol. 139, no. 5, pp. 2475-2481, 2016. 
[106] K. W. Ferrara and J. Foiret, "Spatial and temporal control of hyperthermia using real time ultrasonic thermal strain imaging with motion compensation, Phantom Study," PLOS ONE, vol. 10, p. 1-22, 2015.

[107] K. M. Langen and D. T. Jones, "Organ motion and its management," International Journal of Radiation Oncology Biology Physics, vol. 50, pp. 265-278, 2001.

[108] G. t. Haar, "Therapeutic applications of ultrasound," Progress in Biophysics \& Molecular Biology, vol. 93, no. 1, pp. 111-129, 2007. 